\title{
Substituted Diarylmethylamines by Stereospecific Intramolecular Electrophilic Arylation of Lithiated Ureas
}

\author{
Jonathan Clayden, Jérémy Dufour, Damian Grainger and Madeleine Helliwell
}

\section{SUPPLEMENTARY INFORMATION}

\author{
S1-S67 Copies of ${ }^{1} \mathrm{H}$ and ${ }^{13} \mathrm{C}$ NMR spectra \\ S68-S80 Copies of HPLC traaces for establishing enantiomeric purity \\ S81-S86 General experimental procedure \\ S87-S121 Experimental data for all new compounds
}

Proton nuclear magnetic resonance ( ${ }^{1} \mathrm{H}$ NMR) spectra were recorded on a Varian Gemini $200(200 \mathrm{MHz})$, Varian XL 300 (300 MHz) or a Bruker Ultrashield 500 (500 MHz) spectrometer with residual non-deuterated solvent as the internal standard. Carbon nuclear magnetic resonance $\left({ }^{13} \mathrm{C}\right.$ NMR) spectra were recorded on a Varian XL 300 (75 $\mathrm{MHz})$ spectrometer or a Bruker Ultrashield $500(125 \mathrm{MHz})$. NMR data are presented as follows: chemical shift $\delta$ (in parts per million (ppm) down-field from tetramethylsilane), integration, multiplicity, coupling constant $J$ (in $\mathrm{Hz}$ ) and assignment (based on chemical shift, integration, coupling pattern and COSY, DEPT and HMQC NMR experiments when necessary). Splitting patterns are abbreviated as follows: singlet (s), doublet (d), triplet ( $(\mathrm{t})$, quartet (q), quintet (qn), septet (sept), multiplet (m), broad (br), or a combination of these. The solvent used was deuterated chloroform, dichloromethane or methanol.

Analytical HPLC was carried out on a $(R, R)$-Whelk-O1, $25 \mathrm{~cm} \times 4.6 \mathrm{~mm}$ ID column at room temperature using a Merck Hitachi L-6200 Intelligent pump, typically eluting with $10 \%$ IPA in hexane, flow rate $1 \mathrm{~mL} / \mathrm{min}, 50 \mu \mathrm{L}$ injection (sample concentration of 1 $\mathrm{mg} / \mathrm{mL}$ ). Detection was carried out using a Merck L-300 Photo Diode Array System at UV absorbance at $254 \mathrm{~nm}$. 
Low resolution mass spectra (EI and CI) were recorded on a Fisons VG Trio 2000 quadrupole mass spectrometer. High resolution mass spectra (accurate mass measurement) were recorded on a Thermo Finnigan MAT 95XP mass spectrometer, and are accurate to $\pm 0.001 \mathrm{Da}$.

Infrared spectra were recorded on an ATi Perkin Elmer Spectrum RX1 FTIR spectrometer and only absorption maxima $\left(v_{\max }\right)$ of interest are reported and quoted as wavenumbers $\left(\mathrm{cm}^{-1}\right)$. All samples were run as evaporated films or Nujol mulls on a sodium chloride plate.

Melting points were determined on a Kofler microscope melting point machine and are uncorrected.

Microanalyses were carried out using Carlo-Erba automatic analyser by members of staff of the University of Manchester.

Optical rotations $[\alpha]^{\mathrm{T}}{ }_{\mathrm{D}}$ were measured with a Perkin-Elmer 241 Polarimeter using a cell with a pathlength of $0.25 \mathrm{dm}$. Concentrations $(c)$ are given in grams per $100 \mathrm{ml}$.

Analytical TLC was carried out on pre-coated $\mathrm{UV}_{254}$ plates (Macherey-Nagel alugram. Sil G/UV254), with visualisation by UV light at $254 \mathrm{~nm}$, potassium permanganate dip or dodecamolybdophosphoric acid (PMA) dip.

Flash column chromatography ${ }^{1}$ was carried out using Fluorochem Davisil $40-63 \mu \mathrm{m}$ $60 \AA$ silica, under a positive pressure by means of compressed air, followed by removal of the solvent under reduced pressure after purification.

All reactions were conducted in dried glassware with magnetic stirring under a nitrogen atmosphere unless otherwise stated. 
Reagents and solvents were purified by standard means. ${ }^{2}$ Tetrahydrofuran (THF) and diethyl ether were distilled from sodium wire and benzophenone under nitrogen. Dichloromethane (DCM) and toluene were obtained by distillation from calcium hydride under nitrogen. Triethylamine was distilled from and stored over potassium hydroxide. Anhydrous dimethylformamide was obtained by distillation from calcium sulfate under reduced pressure. DMPU was distilled, under reduced pressure, from calcium hydride, and stored over molecular sieves. All other chemicals were used as received, except where otherwise noted in the experimental text.

Petrol refers to the fraction of light petroleum ether boiling between $40{ }^{\circ} \mathrm{C}$ and $60{ }^{\circ} \mathrm{C}$. All other solvents and commercially obtained reagents were used as received or purified using standard procedures. $s$-Butyllithium was obtained as a solution in cyclohexane. All the above organolithium solutions were titrated prior to use against a solution of diphenylacetic acid. Cooling baths used are acetone/dry ice for $-78{ }^{\circ} \mathrm{C}$ and acetonitrile/dry ice for $-42{ }^{\circ} \mathrm{C}$.

All experiments were performed in anhydrous conditions under an atmosphere of nitrogen, unless otherwise noted in the experimental text. Apparatus was oven-dried and standard techniques were employed in handling air-sensitive materials.

General procedure 1 - Preparation of ureas from isocyanates ${ }^{3}$

Ureas were prepared according to the method of Clayden et al. ${ }^{3}$ The benzylic amine (1 equiv.) was added in one portion to a solution of the aryl isocyanate (1 equiv.) in anhydrous DCM $(0.25 \mathrm{M})$ stirred at room temperature. The solution was stirred for 3-24 hours and the white precipitate filtered and washed with cold DCM and dried under high vacuum. 
General Procedure 2 - Preparation of methylated ureas from ureas

General Procedure $2 a$ - Preparation of methylated ureas using THF

Methylated ureas were prepared according to the method of Clayden et al. ${ }^{3}$ A suspension of urea (1 equiv.) in THF ( $0.15 \mathrm{M})$ was slowly added to a suspension of $\mathrm{NaH}(60 \%$ in mineral oil, 1.2 (mono-methylation) or 2.5 equiv. (di-methylation)) in anhydrous THF. Hydrogen gas was evolved. The mixture was stirred for 15 minutes and MeI (1.5 (monomethylation) or 3.0 equiv. (dimethylation)) was added and the solution stirred for a further 18 hours. Water was added, MeI and THF were evaporated under reduced pressure and the residue was diluted with diethyl ether. The aqueous layer was extracted twice with diethyl ether. The combined organic layers were dried over $\mathrm{MgSO}_{4}$ and concentrated to give the crude urea which was purified by flash column chromatography on silica.

General Procedure $2 b$ - Preparation of methylated ureas using DMF

$\mathrm{NaH}$ (60\% in mineral oil, 2 equiv. (mono-methylation) or 4 equiv. (dimethylation)) was added to a stirred solution of urea in $\operatorname{DMF}(0.2 \mathrm{M})$ at room temperature. After $30 \mathrm{~min}$ methyl iodide ( 2.5 equiv. (mono-methylation) or 5 equiv. (dimethylation)) was added and the reaction left to stir for $24 \mathrm{~h}$. Water was added and the reaction mixture was extracted with diethyl ether. The organic layers were then repeatedly washed with water, dried with $\mathrm{Na}_{2} \mathrm{SO}_{4}$, filtered and evaporated under reduced pressure. The crude product was purified by flash column chromatography on silica.

\section{General Procedure 3 - Lithiation of ureas}

General Procedure $3 a$ - Lithiation of ureas quenching with MeI

A solution of $s$-BuLi (1.3 M in cyclohexane, 2.5 equiv.) was added dropwise to a precooled solution $\left(-78{ }^{\circ} \mathrm{C}\right)$ of urea in anhydrous THF. The resulting reaction mixture was stirred for 30 minutes at this temperature to afford a coloured solution. MeI (2.5 equiv.) was added and the reaction mixture was stirred for a further 1 hour and allowed to warm to room temperature. Water was added, MeI and THF were evaporated under reduced 
pressure and the remaining mixture was partitioned between diethyl ether and water. The aqueous layer was extracted with diethyl ether and the ethereal layers washed with water. The organic layers were dried over $\mathrm{MgSO}_{4}$ and concentrated under reduced pressure to give a residue which was purified by flash column chromatography.

General Procedure $3 b$ - Lithiation of ureas quenching with water

A solution of $s$-BuLi (1.3 M in cyclohexane, 2.5 equiv.) was added dropwise to a precooled solution $\left(-78{ }^{\circ} \mathrm{C}\right)$ of urea in anhydrous THF $(0.07 \mathrm{M})$. The resulting reaction mixture was stirred for 0.5 or $2 \mathrm{~h}$ at this temperature to afford a coloured solution. Water was added and the reaction mixture stirred for a further 30 minutes while warming to room temperature. The mixture was partitioned between diethyl ether and water. The aqueous layer was extracted with diethyl ether and the combined organic layers washed with water, dried over $\mathrm{MgSO}_{4}$ and concentrated under reduced pressure to give the crude product which was purified by flash column chromatography.

\section{General Procedure $3 c$ - Lithiation of ureas in the presence DMPU}

A solution of $s$-BuLi (1.3 M in cyclohexane, 2.5 equiv.) was added dropwise to a precooled solution $\left(-78{ }^{\circ} \mathrm{C}\right)$ of urea in a mixture of anhydrous THF and DMPU (4:1, 0.07 M). The resulting reaction mixture was stirred for 2 or $6 \mathrm{~h}$ after which MeOD was added and the reaction mixture stirred for a further 30 minutes while warming to room temperature. THF was evaporated under reduced pressure and the remaining mixture partitioned between diethyl ether and water. The organic layer was washed 3 times with water and the combined organic layers were dried over $\mathrm{Na}_{2} \mathrm{SO}_{4}$ and concentrated under reduced pressure to give a residue which was purified by flash column chromatography.

General Procedure $3 d$ - Lithiation of ureas in the presence DMPU and quenching with $t$ $\mathrm{BuONO}$

A solution of $s$-BuLi (1.3 M in cyclohexane, 2.5 equiv.) was added dropwise to a precooled solution $\left(-78{ }^{\circ} \mathrm{C}\right)$ of urea in anhydrous THF and DMPU $(4: 1,0.07 \mathrm{M})$. The mixture was stirred for $6 \mathrm{~h}$ after which $t$-BuONO ( 6 equiv.) was addedThe mixture was allowed to warm to room temperature overnight and partitioned between diethyl ether 
and a saturated aqueous solution of $\mathrm{K}_{2} \mathrm{CO}_{3}$. The organic layer was washed 3 times with saturated aqueous $\mathrm{K}_{2} \mathrm{CO}_{3}$, dried over $\mathrm{Na}_{2} \mathrm{SO}_{4}$, filtered and concentrated under reduced pressure to give a residue which was purified by flash column chromatography.

General Procedure $3 e$ - Lithiation of ureas in the presence DMPU and quenching with air

A solution of $s$-BuLi (1.3 M in cyclohexane, 2.5 equiv.) was added dropwise to a precooled solution $\left(-78^{\circ} \mathrm{C}\right)$ of urea in mixture of anhydrous THF and DMPU $(4: 1,0.07 \mathrm{M})$. The mixture was stirred for $2 \mathrm{~h}$ and a stream of air was bubbled through the reaction mixture for $0.5 \mathrm{~h}$. The reaction was allowed to warm to room temperature. THF was evaporated under reduced pressure and the remaining mixture partitioned between diethyl ether and water. The organic layer was washed 3 times with water and the combined organic layers were dried over $\mathrm{Na}_{2} \mathrm{SO}_{4}$ and concentrated under reduced pressure to give a residue which was purified by flash column chromatography.

\section{General Procedure 4 - Synthesis of N-nitroso ureas}

A mixture of the urea in $\mathrm{CH}_{2} \mathrm{Cl}_{2}(0.15 \mathrm{M})$ and $t$-BuONO (6 equiv.) was stirred at room temperature for $24 \mathrm{~h}$ and partitioned between diethyl ether and a saturated aqueous solution of $\mathrm{K}_{2} \mathrm{CO}_{3}$. The organic layer was washed 5 times with saturated aqueous $\mathrm{K}_{2} \mathrm{CO}_{3}$ and dried over $\mathrm{Na}_{2} \mathrm{SO}_{4}$, filtered and concentrated under reduced pressure to give a residue which was purified by flash column chromatography.

\section{General Procedure 5 - Hydrolysis of N-nitroso ureas}

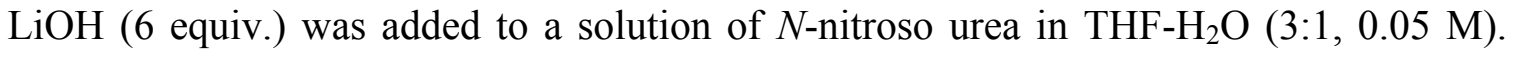
The mixture was heated to $80^{\circ} \mathrm{C}$ for 2 or 3 days and partitioned between diethyl ether and $\mathrm{H}_{2} \mathrm{O}$. The organic layer was washed twice with $\mathrm{H}_{2} \mathrm{O}$ and then brine, dried over $\mathrm{Na}_{2} \mathrm{SO}_{4}$, filtered and concentrated under reduced pressure to give a residue which was purified by flash column chromatography. 


\section{1-Benzyl-3-(2,6-dimethylphenyl)-3-methyl-1-phenylurea 1a}

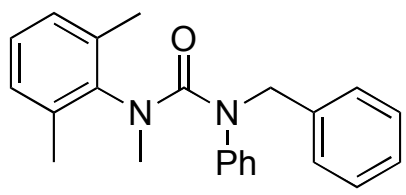

General procedures 1 and $2 \mathrm{a}$ were followed. Flash column chromatography $\left(\mathrm{SiO}_{2} ; 1: 1\right.$ Petrol:EtOAc) gave the title compound as a white solid $(3.0 \mathrm{~g}, 96 \%)$; m.p. $89-90{ }^{\circ} \mathrm{C}$ (Petrol-EtOAc); Rf 0.32 (Petrol:EtOAc, 4:1); IR $\boldsymbol{v}_{\max }\left(\mathrm{CHCl}_{3}\right) / \mathrm{cm}^{-1} 1641(\mathrm{C}=\mathrm{O})$; ${ }^{\mathbf{1}} \mathbf{H}$ NMR $\left(300 \mathrm{MHz}, \mathrm{CDCl}_{3}\right) \delta 7.23(\mathrm{~m}, 5 \mathrm{H}), 6.96-6.80(\mathrm{~m}, 4 \mathrm{H}), 6.75-6.69(\mathrm{~m}, 2 \mathrm{H}), 6.50-$ $6.43(\mathrm{~m}, 2 \mathrm{H}), 4.75(\mathrm{~s}, 2 \mathrm{H}), 3.11(\mathrm{~s}, 3 \mathrm{H}), 1.96(\mathrm{~s}, 6 \mathrm{H}) ;{ }^{13} \mathbf{C}$ NMR $\left(75 \mathrm{MHz}, \mathrm{CDCl}_{3}\right) \delta$ $161.3(\mathrm{C}), 143.0(\mathrm{C}), 142.2$ (C), 138.4 (C), 136.1 (C), $130.0(\mathrm{CH}), 128.5(\mathrm{CH}), 128.6$ $(\mathrm{CH}), 128.4(\mathrm{CH}), 127.9(\mathrm{CH}), 127.4(\mathrm{CH}), 126.9(\mathrm{CH}), 126.2(\mathrm{CH}), 56.6\left(\mathrm{CH}_{2}\right), 37.5$ $\left(\mathrm{CH}_{3}\right), 18.8\left(\mathrm{CH}_{3}\right)$; LRMS (CI+) $m / z 345\left(\mathrm{M}+\mathrm{H}^{+}, 38\right)$; HRMS (EI+): calcd for $\mathrm{C}_{23} \mathrm{H}_{25} \mathrm{~N}_{2} \mathrm{O}\left(\mathrm{M}+\mathrm{H}^{+}\right): 345.1961$, found: 345.1953 [ $\Delta-2.3 \mathrm{ppm}$ ]; $\mathbf{E A}$ found: $\mathrm{C} 80.56 \%, \mathrm{H}$ $7.19 \%, \mathrm{~N} 8.14 \% . \mathrm{C}_{23} \mathrm{H}_{24} \mathrm{~N}_{2} \mathrm{O}$ requires $\mathrm{C} 80.20 \%, \mathrm{H} 7.02 \%, \mathrm{~N} 8.13 \%$.

\section{1-Benzyl-3-methyl-1-phenyl-3-p-tolylurea $1 \mathrm{~b}$}

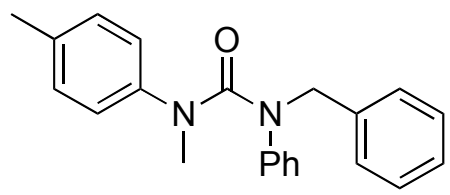

General procedures 1 and $2 \mathrm{a}$ were followed. Flash column chromatography $\left(\mathrm{SiO}_{2} ; 1: 1\right.$ Petrol:EtOAc) gave the title compound as a white solid $(2.3 \mathrm{~g}, 93 \%)$; m.p. $66-68{ }^{\circ} \mathrm{C}$ (Petrol-EtOAc); Rf 0.45 (Petrol:EtOAc, 4:1); IR $\boldsymbol{V}_{\max }\left(\mathrm{CH}_{2} \mathrm{Cl}_{2}\right) / \mathrm{cm}^{-1} 1655(\mathrm{C}=\mathrm{O})$; ${ }^{1} \mathbf{H}$ NMR $\left(500 \mathrm{MHz}, \mathrm{CDCl}_{3}\right) \delta$ 7.34-7.16 (m, 5H), 7.02-6.74 (m, 5H), 6.70-6.56 (m, 4H), 4.78 (s, 2H), 3.12 (s, 3H), 2.20 (s, 3H); ${ }^{13}$ C NMR (125 MHz, $\left.\mathrm{CDCl}_{3}\right) \delta 161.7$ (C), 144.9 (C), $143.0(\mathrm{C}), 139.0(\mathrm{C}), 135.0(\mathrm{C}), 129.5(\mathrm{CH}), 129.1(\mathrm{CH}), 128.8(\mathrm{CH}), 128.6(\mathrm{CH})$, $127.4(\mathrm{CH}), 127.1(\mathrm{CH}), 126.4(\mathrm{CH}), 125.4(\mathrm{CH}), 55.7\left(\mathrm{CH}_{2}\right), 40.1\left(\mathrm{CH}_{3}\right), 21.2\left(\mathrm{CH}_{3}\right)$; LRMS (CI+) m/z $331\left(100 \%, \mathrm{M}+\mathrm{H}^{+}\right)$; HRMS (EI+): calcd for $\mathrm{C}_{22} \mathrm{H}_{23} \mathrm{~N}_{2} \mathrm{O}_{1}\left(\mathrm{M}+\mathrm{H}^{+}\right)$: requires 331.1805, found: 331.1808 [ $\Delta 0.9 \mathrm{ppm}]$. 


\section{1-Benzyl-3-methyl-3-phenyl-1-p-tolylurea 1c}

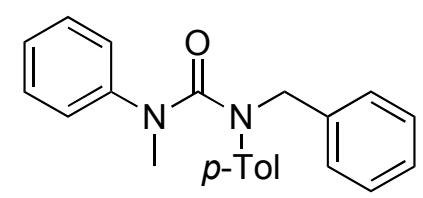

General procedures 1 ( $N$-methyl aniline with $p$-tolyl isocyanate) and 2a (using benzyl bromide) were followed. Flash column chromatography $\left(\mathrm{SiO}_{2} ; 1: 1\right.$ Petrol:EtOAc) gave the title compound as a white solid (2.5 g, 87\%); m.p. $60-61{ }^{\circ} \mathrm{C}$ (Petrol-EtOAc); Rf 0.45 (Petrol:EtOAc, 4:1); IR $v_{\max }\left(\mathrm{CH}_{2} \mathrm{Cl}_{2}\right) / \mathrm{cm}^{-1} 1655(\mathrm{C}=\mathrm{O}) ;{ }^{1} \mathbf{H} \mathbf{N M R}\left(500 \mathrm{MHz}, \mathrm{CDCl}_{3}\right) \delta$ 7.34-7.20 (m, 5H), 7.08-6.90 (m, 3H), 6.80-6.70 (m, 4H), 6.58-6.48 (m, 2H), $4.76(\mathrm{~s}$, 2H), 3.18 (s, 3H), 2.18 (s, 3H); ${ }^{13} \mathbf{C}$ NMR (125 MHz, $\left.\mathrm{CDCl}_{3}\right) \delta 161.7$ (C), 146.1 (C), $142.0(\mathrm{C}), 139.0(\mathrm{C}), 135.0(\mathrm{C}), 129.4(\mathrm{CH}), 129.1(\mathrm{CH}), 128.9(\mathrm{CH}), 128.6(\mathrm{CH}), 127.4$ $(\mathrm{CH}), 127.0(\mathrm{CH}), 126.4(\mathrm{CH}), 125.2(\mathrm{CH}), 55.7\left(\mathrm{CH}_{2}\right), 40.1\left(\mathrm{CH}_{3}\right), 21.2\left(\mathrm{CH}_{3}\right)$; LRMS $(\mathrm{CI}+) \mathrm{m} / z 331\left(100 \%, \mathrm{M}+\mathrm{H}^{+}\right)$; HRMS (EI+): calcd for $\mathrm{C}_{22} \mathrm{H}_{23} \mathrm{~N}_{2} \mathrm{O}_{1}\left(\mathrm{M}+\mathrm{H}^{+}\right): 331.1805$, found: 331.1799 [ $\Delta-1.8 \mathrm{ppm}]$.

\section{1-Benzyl-1,3-dimethyl-3-phenylurea 1d}

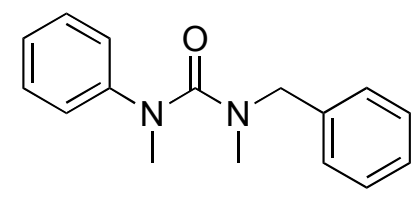

General procedures 1 and $2 \mathrm{a}$ were followed. Flash column chromatography $\left(\mathrm{SiO}_{2} ; 4: 1\right.$ Petrol:EtOAc) gave the title compound as a pale yellow oil (3.0 g, 94\%); $\mathbf{R}_{\mathbf{f}} 0.37$ (Petrol:EtOAc, 2:1); IR $v_{\max }\left(\mathrm{CHCl}_{3}\right) / \mathrm{cm}^{-1} 1649(\mathrm{C}=\mathrm{O}) ;{ }^{1} \mathbf{H}$ NMR $\left(300 \mathrm{MHz}, \mathrm{CDCl}_{3}\right) \delta$ 7.40-7.22 (m, 7H), 7.19-7.06 (m, 3H), 4.42 (s, 2H), 3.29 (s, 3H), 2.53 (s, 3H); ${ }^{13}$ C NMR $\left(75 \mathrm{MHz}, \mathrm{CDCl}_{3}\right) \delta 162.3(\mathrm{C}), 147.1(\mathrm{C}), 137.9(\mathrm{C}), 129.7(\mathrm{CH}), 128.7(\mathrm{CH}), 128.3$ $(\mathrm{CH}), 127.5(\mathrm{CH}), 124.7(\mathrm{CH}), 124.2(\mathrm{CH}), 53.8\left(\mathrm{CH}_{2}\right), 40.2\left(\mathrm{CH}_{3}\right), 36.3\left(\mathrm{CH}_{3}\right)$; LRMS $(\mathrm{CI}+) m / z 255\left(\mathrm{M}+\mathrm{H}^{+}, 100\right)$; HRMS (EI+): calcd for $\mathrm{C}_{16} \mathrm{H}_{19} \mathrm{~N}_{2} \mathrm{O}\left(\mathrm{M}+\mathrm{H}^{+}\right): 255.1492$, found: $255.1502[\Delta 3.9 \mathrm{ppm}]$. 


\section{1-Benzyl-1,3-dimethyl-3-o-tolylurea 1e}

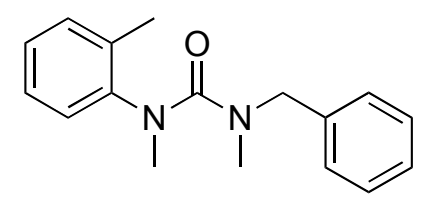

General procedures 1 and $2 \mathrm{a}$ were followed. Flash column chromatography $\left(\mathrm{SiO}_{2} ; 2: 1\right.$ Petrol:EtOAc) gave the title compound as a yellow oil $(2.0 \mathrm{~g}, 94 \%) ; \mathbf{R}_{\mathbf{f}} 0.42$

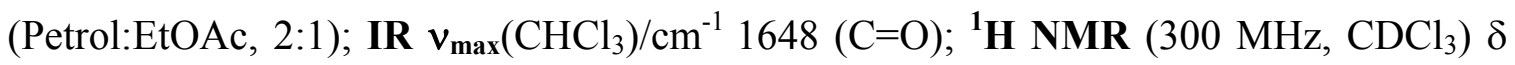
7.37-7.05 (m, 9H), 4.37 (s, 2H), 3.14 (s, 3H), 2.41 (s, 3H), 2.32 (s, 3H); ${ }^{13}$ C NMR (75 $\left.\mathrm{MHz} \mathrm{CDCl}_{3}\right) \delta 163.1(\mathrm{C}), 145.6(\mathrm{C}), 138.2(\mathrm{C}), 134.5(\mathrm{C}), 131.8(\mathrm{CH}), 128.7(\mathrm{CH})$, $128.2(\mathrm{CH}), 127.5(\mathrm{CH}), 127.4(\mathrm{CH}), 127.0(\mathrm{CH}), 126.7(\mathrm{CH}), 53.8\left(\mathrm{CH}_{2}\right), 39.5\left(\mathrm{CH}_{3}\right)$, $35.7\left(\mathrm{CH}_{3}\right), 18.0\left(\mathrm{CH}_{3}\right)$; LRMS (CI+) $m / z 269\left(100 \%, \mathrm{M}+\mathrm{H}^{+}\right)$; HRMS (EI+): calcd for $\mathrm{C}_{17} \mathrm{H}_{21} \mathrm{~N}_{2} \mathrm{O}\left(\mathrm{M}+\mathrm{H}^{+}\right)$: 269.1648. found: 269.1645 [ $\left.\Delta-1.3 \mathrm{ppm}\right]$.

\section{1-Benzyl-3-(2-methoxyphenyl)-1,3-dimethylurea $1 f$}<smiles>COc1ccccc1N(C)C(=O)N(C)Cc1ccccc1</smiles>

General procedures 1 and $2 \mathrm{a}$ were followed. Flash column chromatography $\left(\mathrm{SiO}_{2} ; 2: 1\right.$ Petrol:EtOAc) gave the title compound as a pale yellow oil (4.0 g, 84\%); Rf 0.45 (Petrol:EtOAc, 2:1); IR $\boldsymbol{v}_{\max }\left(\mathrm{CHCl}_{3}\right) / \mathrm{cm}^{-1} 1645(\mathrm{C}=\mathrm{O}) ;{ }^{1} \mathbf{H} \mathbf{N M R}\left(300 \mathrm{MHz}, \mathrm{CDCl}_{3}\right) \delta$ 7.35-7.17 (m, 6H), 7.12-7.07 (m, 1H), 6.98-6.90 (m, 2H), $4.40(\mathrm{~s}, 2 \mathrm{H}), 3.90(\mathrm{~s}, 3 \mathrm{H}), 3.16$ (s, 3H), 2.42 (s, 3H); ${ }^{13}$ C NMR (75 MHz, $\left.\mathrm{CDCl}_{3}\right) \delta 163.1$ (C), 154.3 (C), 138.3 (C), $135.2(\mathrm{C}), 128.6(\mathrm{CH}), 128.2(\mathrm{CH}), 127.9(\mathrm{CH}), 127.6(\mathrm{CH}), 127.3(\mathrm{CH}), 121.1(\mathrm{CH})$, $112.1(\mathrm{CH}), 55.8\left(\mathrm{CH}_{3}\right), 53.8\left(\mathrm{CH}_{2}\right), 38.8\left(\mathrm{CH}_{3}\right), 35.8\left(\mathrm{CH}_{3}\right)$; LRMS (CI+) $m / z 285$ $\left(\mathrm{M}+\mathrm{H}^{+}, 100\right)$; HRMS (EI+): calcd for $\mathrm{C}_{17} \mathrm{H}_{21} \mathrm{~N}_{2} \mathrm{O}_{2}\left(\mathrm{M}+\mathrm{H}^{+}\right)$285.1598. found: 285.1604 $[\Delta 2.1 \mathrm{ppm}]$. 


\section{1-Benzyl-3-(2,6-dimethylphenyl)-1,3-dimethylurea $1 \mathrm{~g}$}

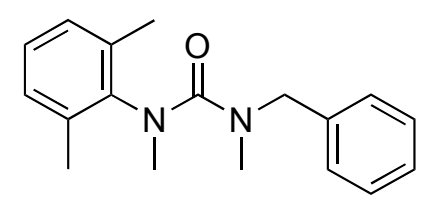

General procedures 1 and $2 \mathrm{a}$ were followed. Flash column chromatography $\left(\mathrm{SiO}_{2} ; 1: 1\right.$ Petrol:EtOAc) gave the title compound as a colourless oil (2.0 g, 94\%); Rf 0.17

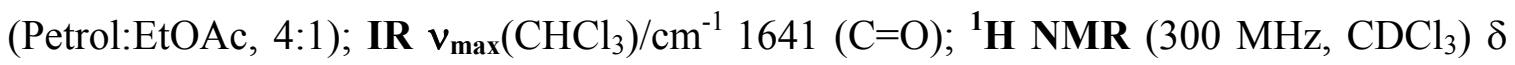
7.35-7.11 (m, 5H), 7.08 (brs, 3H), 4.30 (s, 2H), 3.14 (s, 3H), 2.41 (s, 3H), 2.27 (s, 6H); ${ }^{13}$ C NMR (75 MHz, $\left.\mathrm{CDCl}_{3}\right) \delta 162.7$ (C), 143.5 (C), 138.3 (C), 135.8 (C), $129.4(\mathrm{CH})$, $128.6(\mathrm{CH}), 128.1(\mathrm{CH}), 127.3(\mathrm{CH}), 127.1(\mathrm{CH}), 54.0\left(\mathrm{CH}_{2}\right), 38.0\left(\mathrm{CH}_{3}\right), 35.4\left(\mathrm{CH}_{3}\right)$, $18.3\left(\mathrm{CH}_{3}\right)$; LRMS (CI+) $m / z 283\left(\mathrm{M}+\mathrm{H}^{+}, 100\right)$; HRMS (EI+): calcd for $\mathrm{C}_{18} \mathrm{H}_{23} \mathrm{~N}_{2} \mathrm{O}$ $\left(\mathrm{M}+\mathrm{H}^{+}\right): 283.1805$, found: 283.1807 [ $\left.\Delta 0.7 \mathrm{ppm}\right]$.

\section{1-Benzyl-3-(4-chlorophenyl)-1,3-dimethylurea $1 \mathrm{~h}$}

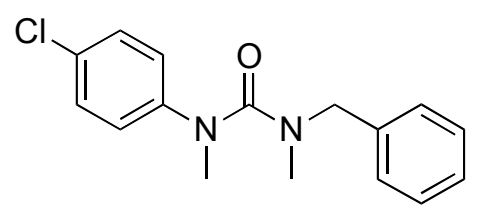

General procedures 1 and $2 \mathrm{a}$ were followed. Flash column chromatography $\left(\mathrm{SiO}_{2} ; 1: 1\right.$ Petrol:EtOAc) gave the title compound as a pale yellow oil (3.1 g, 91\%); Rf 0.46 (Petrol:EtOAc, 1:1); IR $v_{\max }\left(\mathrm{CHCl}_{3}\right) / \mathrm{cm}^{-1} 1652(\mathrm{C}=\mathrm{O}) ;{ }^{1} \mathbf{H}$ NMR $\left(300 \mathrm{MHz}, \mathrm{CDCl}_{3}\right) \delta$ 7.44-7.30 (m, 7H), 7.27-7.22 (m, 2H), 4.42 (s, 2H), 3.27 (s, 3H), 2.56 (s, 3H); ${ }^{13} \mathbf{C}$ NMR $\left(75 \mathrm{MHz}, \mathrm{CDCl}_{3}\right) \delta 162.0(\mathrm{C}), 145.6(\mathrm{C}), 137.7(\mathrm{C}), 129.9(\mathrm{C}), 129.8(\mathrm{CH}), 128.8(\mathrm{CH})$, $128.3(\mathrm{CH}), 127.7(\mathrm{CH}), 125.2(\mathrm{CH}), 53.8\left(\mathrm{CH}_{2}\right), 40.0\left(\mathrm{CH}_{3}\right), 36.4\left(\mathrm{CH}_{3}\right)$; LRMS (CI+) $m / z 289\left(\mathrm{M}+\mathrm{H}^{+}, 100\right)$; HRMS (EI+): calcd for $\mathrm{C}_{16} \mathrm{H}_{18} \mathrm{~N}_{2} \mathrm{O}^{35} \mathrm{Cl}\left(\mathrm{M}\left({ }^{35} \mathrm{Cl}\right)+\mathrm{H}^{+}\right)$289.1102, found: $289.1102[\Delta-0.1 \mathrm{ppm}]$. 


\section{1-Benzyl-3-(4-methoxyphenyl)-1,3-dimethylurea 1i}

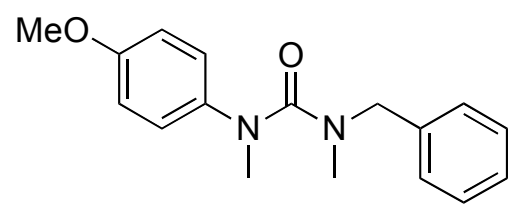

General procedures 1 and $2 \mathrm{a}$ were followed. Flash column chromatography $\left(\mathrm{SiO}_{2} ; 1: 1\right.$ Petrol:EtOAc) gave the title compound as a colourless oil $(2.35 \mathrm{~g}, 86 \%)$; Rf 0.46 (Petrol:EtOAc, 2:1); IR $v_{\max }\left(\mathrm{CH}_{3} \mathrm{Cl}\right) / \mathrm{cm}^{-1} 1647(\mathrm{C}=\mathrm{O}) ;{ }^{1} \mathbf{H}$ NMR $\left(300 \mathrm{MHz}, \mathrm{CDCl}_{3}\right) \delta$ 7.38-7.23 (m, 5H), 7.09-7.04 (m, 2H), 6.98-6.90 (m, 2H), $4.44(\mathrm{~s}, 2 \mathrm{H}), 3.83(\mathrm{~s}, 3 \mathrm{H}), 3.24$ $(\mathrm{s}, 3 \mathrm{H}), 2.52(\mathrm{~s}, 3 \mathrm{H}) ;{ }^{13} \mathbf{C}$ NMR $\left(75 \mathrm{MHz}, \mathrm{CDCl}_{3}\right) \delta 160.5(\mathrm{C}), 157.0(\mathrm{C}), 140.1(\mathrm{C})$, $138.1(\mathrm{C}), 128.6(\mathrm{CH}), 128.2(\mathrm{CH}), 127.3(\mathrm{CH})$ 126.1(CH), $114.9(\mathrm{CH}), 55.5\left(\mathrm{CH}_{3}\right), 53.7$ $\left(\mathrm{CH}_{2}\right), 40.8\left(\mathrm{CH}_{3}\right), 36.3\left(\mathrm{CH}_{3}\right)$; LRMS $(\mathrm{CI}+) \mathrm{m} / z 284\left(\mathrm{M}^{+}, 100\right)$; HRMS (EI+): calcd for $\mathrm{C}_{17} \mathrm{H}_{21} \mathrm{~N}_{2} \mathrm{O}_{2}\left(\mathrm{M}+\mathrm{H}^{+}\right)$285.1598, found: 285.1600 [ $\left.\Delta 0.7 \mathrm{ppm}\right]$.

\section{1-[(2,6-Dimethylphenyl)-4-methylcyclohexa-2,5-dienylidenemethyl]-3,3-dimethyl-1-}

\section{phenylurea 2}

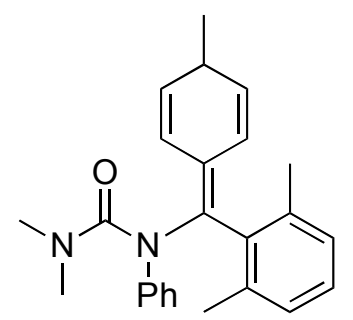

General procedure 3a was followed using 1a $(200 \mathrm{mg}, 0.58 \mathrm{mmol}), s$-BuLi $(1.3 \mathrm{M}$ solution in cyclohexane, $1.12 \mathrm{ml}, 1.45 \mathrm{mmol})$ and $\mathrm{MeI}(0.04 \mathrm{ml}, 0.64 \mathrm{mmol}, 1.1$ equiv.) in $10 \mathrm{ml}$ of anhydrous THF to give a crude product. Purification by flash column chromatography $\left(\mathrm{SiO}_{2} ; 20: 1\right.$ to 10:1 Petrol:toluene) gave the unstable title compound (30 mg, 14\%) as an indigo oil; Rf 0.43 (toluene:EtOAc, 6:1); IR $v_{\max }\left(\mathrm{CHCl}_{3}\right) / \mathrm{cm}^{-1} 1668$ $(\mathrm{C}=\mathrm{O}) ;{ }^{1} \mathbf{H}$ NMR $\left(300 \mathrm{MHz}, \mathrm{CDCl}_{3}\right) \delta$ 7.16-7.09 (m, 2H), 6.97-6.85 (m, 6H), 6.20 (dt, $J$ $=10,1.8 \mathrm{~Hz}, 1 \mathrm{H}), 5.85(\mathrm{ddd}, J=10,4,2 \mathrm{~Hz}, 1 \mathrm{H}), 5.70(\mathrm{dt}, J=10,2 \mathrm{~Hz}, 1 \mathrm{H}), 5.63$ (ddd, $J=10,4,2 \mathrm{~Hz}, 1 \mathrm{H}), 3.20-3.09(\mathrm{~m}, 1 \mathrm{H}), 2.76(\mathrm{~s}, 6 \mathrm{H}), 2.38(\mathrm{~s}, 6 \mathrm{H}), 1.16(\mathrm{~d}, J=7$ $\mathrm{Hz}, 3 \mathrm{H}) ;{ }^{13} \mathbf{C}$ NMR (75 MHz, $\left.\mathrm{CDCl}_{3}\right) \delta 159.6$ (C), 143.9 (C), 139.3 (C), 139.0 (C), 135.0 (C), $134.4(\mathrm{CH}), 132.8(\mathrm{C}), 132.6(\mathrm{CH}), 128.8(\mathrm{CH}), 128.5(\mathrm{CH}), 127.8(\mathrm{CH}), 127.7$ $(\mathrm{CH}), 127.6(\mathrm{CH}), 125.2(\mathrm{CH}), 123.9(\mathrm{CH}), 123.5(\mathrm{CH}), 123.4(\mathrm{CH}), 122.6(\mathrm{CH}), 38.1$ 
$\left(\mathrm{CH}_{3}\right), 33.9(\mathrm{CH}), 22.2\left(\mathrm{CH}_{3}\right), 21.2\left(\mathrm{CH}_{3}\right), 21.1\left(\mathrm{CH}_{3}\right)$; LRMS $(\mathrm{CI}+) \mathrm{m} / z 374\left(\mathrm{M}+\mathrm{H}^{+}\right.$, 100), 300 (M-CON(Me) 2,35$), 209$ (30), 72 ( $\left.\mathrm{CON}(\mathrm{Me})_{2}, 55\right)$.

( \pm )-1-((2,6-Dimethylphenyl)(phenyl)methyl)-3-methyl-1-phenylurea 3a

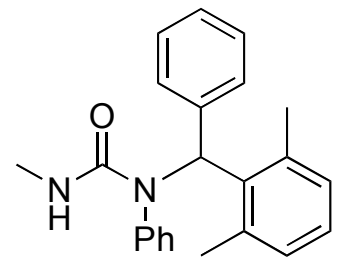

General procedure $3 \mathrm{~b}$ was followed. Flash column chromatography $\left(\mathrm{SiO}_{2} ; 2: 1\right.$ Petrol:EtOAc) gave the title compound as a colourless crystalline solid (268 mg, 89\%). m.p. $177-181^{\circ} \mathrm{C}$; Rf 0.08 (Petrol:EtOAc, 2:1); IR $v_{\max }\left(\mathrm{CHCl}_{3}\right) / \mathrm{cm}^{-1} 3457(\mathrm{~N}-\mathrm{H}), 1659$ $(\mathrm{C}=\mathrm{O}) ;{ }^{1} \mathbf{H}$ NMR $\left(300 \mathrm{MHz}, \mathrm{CDCl}_{3}\right) \delta 7.59(\mathrm{~s}, 1 \mathrm{H}), 7.36-7.31(\mathrm{~m}, 4 \mathrm{H}), 7.25-7.14(\mathrm{~m}$, 4H), 6.93 (t, $J=7 \mathrm{~Hz}, 1 \mathrm{H}), 6.94-6.85(\mathrm{~m}, 4 \mathrm{H}), 4.11(\mathrm{~d}, J=5 \mathrm{~Hz}, 1 \mathrm{H}), 3.03$ (d, $J=5 \mathrm{~Hz}$, 3H), 1.77 (brs, 6H); ${ }^{13}$ C NMR (75 MHz, $\mathrm{CDCl}_{3}$ ) $\delta 158.4$ (C), 143.2 (C), 139.9 (C), 138.9 (C), $136.2(\mathrm{C}), 132.0(\mathrm{CH}), 129.3(\mathrm{CH}), 129.2(\mathrm{CH}), 128.7(\mathrm{CH}), 128.6(\mathrm{CH}), 128.1$ $(\mathrm{CH}), 126.1(\mathrm{CH}), 125.8(\mathrm{CH}), 59.6(\mathrm{CH}), 28.1\left(\mathrm{CH}_{3}\right), 21.8\left(\mathrm{CH}_{3}\right)$; LRMS (CI+) $m / z 345$ $\left(\mathrm{M}+\mathrm{H}^{+}, 100\right), 195\left(\left[\mathrm{C}_{6} \mathrm{H}_{3}(\mathrm{Me})_{2} \mathrm{CHPh}\right]^{+}, 30\right)$; HRMS (EI+): calcd for $\mathrm{C}_{23} \mathrm{H}_{25} \mathrm{~N}_{2} \mathrm{O}$ $\left(\mathrm{M}+\mathrm{H}^{+}\right)$345.1961, found: 345.1959 [ $\left.\Delta-0.5 \mathrm{ppm}\right]$.

\section{( \pm )-3-Methyl-1-phenyl-1-[phenyl(p-tolyl)methyl]urea 3b}

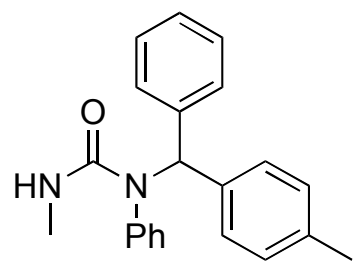

General procedure $3 \mathrm{~b}$ was followed. Flash column chromatography $\left(\mathrm{SiO}_{2} ; 1: 1\right.$ Petrol:EtOAc) gave the title compound as a pale orange solid (176 mg, 85\%). m.p. 108$110^{\circ} \mathrm{C}$; Rf 0.87 (Petrol:EtOAc, 4:1); IR $v_{\max }\left(\mathrm{CH}_{2} \mathrm{Cl}_{2}\right) / \mathrm{cm}^{-1} 3365(\mathrm{~N}-\mathrm{H}), 1658(\mathrm{C}=\mathrm{O}) ;{ }^{1} \mathbf{H}$ NMR $\left(300 \mathrm{MHz}, \mathrm{CDCl}_{3}\right) \delta$ 7.16-7.05 (m, 9H), 6.98-6.94 (m, 4H), 6.83-6.68 (m, 2H), 4.08 (brd, $J=5 \mathrm{~Hz}, 1 \mathrm{H}), 2.69$ (d, $J=5 \mathrm{~Hz}, 3 \mathrm{H}), 2.22$ (s, 3H); ${ }^{13} \mathbf{C}$ NMR $(75 \mathrm{MHz}$, $\left.\mathrm{CDCl}_{3}\right) \delta 158.1(\mathrm{C}), 140.4(\mathrm{C}), 139.5(\mathrm{C}), 137.1(\mathrm{C}), 136.6(\mathrm{C}), 131.0(\mathrm{CH}), 129.4(\mathrm{CH})$, $129.4(\mathrm{CH}), 129.3(\mathrm{CH}), 128.7(\mathrm{CH}), 128.6(\mathrm{CH}), 127.9(\mathrm{CH}), 127.0(\mathrm{CH}), 64.0(\mathrm{CH})$, 
$27.7\left(\mathrm{CH}_{3}\right), 21.1\left(\mathrm{CH}_{3}\right)$; LRMS (CI+) $\mathrm{m} / z 331\left(\mathrm{M}+\mathrm{H}^{+}, 100\right)$; HRMS (EI+): calcd for $\mathrm{C}_{22} \mathrm{H}_{23} \mathrm{~N}_{2} \mathrm{O}_{1}\left(\mathrm{M}+\mathrm{H}^{+}\right)$331.1805, found: 331.1813 [ $\left.\Delta 2.4 \mathrm{ppm}\right]$.

\section{1-Benzhydryl-3-methyl-1-p-tolylurea 3c}<smiles>CNC(=O)N([O-])C(c1ccccc1)c1ccccc1</smiles>

General procedure $3 \mathrm{~b}$ was followed. Flash column chromatography $\left(\mathrm{SiO}_{2} ; 1: 1\right.$ Petrol:EtOAc) gave the title compound as a white solid $(176 \mathrm{mg}, 85 \%)$. m.p. $128-130^{\circ} \mathrm{C}$; Rf 0.87 (Petrol:EtOAc, 4:1); IR $\boldsymbol{v}_{\max }\left(\mathrm{CH}_{2} \mathrm{Cl}_{2}\right) / \mathrm{cm}^{-1} 3374(\mathrm{~N}-\mathrm{H}), 1658(\mathrm{C}=\mathrm{O}) ;{ }^{1} \mathbf{H}$ NMR $\left(300 \mathrm{MHz}, \mathrm{CDCl}_{3}\right) \delta$ 7.11-7.01 (m, 10H), $6.95(\mathrm{~s}, 1 \mathrm{H}), 6.82-6.78(\mathrm{~m}, 2 \mathrm{H}), 6.61-6.56(\mathrm{~m}$, 2H), 4.03 (brd, $J=5 \mathrm{~Hz}, 1 \mathrm{H}), 2.61(\mathrm{~d}, J=5 \mathrm{~Hz}, 3 \mathrm{H}), 2.11(\mathrm{~s}, 3 \mathrm{H}) ;{ }^{13} \mathbf{C}$ NMR $(75 \mathrm{MHz}$, $\left.\mathrm{CDCl}_{3}\right) \delta 158.3(\mathrm{C}), 140.3(\mathrm{C}), 137.9(\mathrm{C}), 136.6(\mathrm{C}), 130.8(\mathrm{CH}), 129.9(\mathrm{CH}), 129.5$ $(\mathrm{CH}), 127.9(\mathrm{CH}), 127.0(\mathrm{CH}), 64.0(\mathrm{CH}), 27.6\left(\mathrm{CH}_{3}\right), 21.1\left(\mathrm{CH}_{3}\right)$; LRMS (CI+) $\mathrm{m} / z 331$ $\left(100 \%, \mathrm{M}+\mathrm{H}^{+}\right)$; HRMS (EI+): calcd for $\mathrm{C}_{22} \mathrm{H}_{23} \mathrm{~N}_{2} \mathrm{O}_{1}\left(\mathrm{M}+\mathrm{H}^{+}\right)$331.1805, found: 331.1802 $[\Delta-0.9 \mathrm{ppm}]$.

\section{1-Benzhydryl-1,3-dimethylurea 3d}

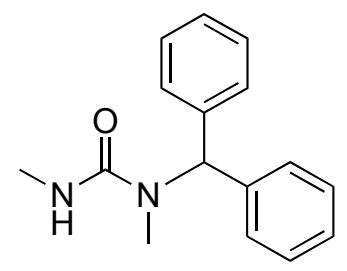

General procedure $3 \mathrm{~b}$ was followed. Flash column chromatography $\left(\mathrm{SiO}_{2} ; 1: 1\right.$ Petrol:EtOAc) gave the title compound as a white solid (155 mg, 78\%). m.p. $146-147{ }^{\circ} \mathrm{C}$; Rf 0.15 (Petrol:EtOAc, 2:1); IR $\boldsymbol{v}_{\max }\left(\mathrm{CHCl}_{3}\right) / \mathrm{cm}^{-1} 3349(\mathrm{~N}-\mathrm{H}), 1629(\mathrm{C}=\mathrm{O}) ;{ }^{\mathbf{1}} \mathbf{H}$ NMR $\left(300 \mathrm{MHz}, \mathrm{CDCl}_{3}\right) \delta$ 7.40-7.28 (m, 6H), 7.27-7.20 (m, 4H), 6.82 (s, 1H), 4.63 (brs, 1H), 2.87 (s, 3H), 2.72 (s, 3H); ${ }^{13} \mathbf{C}$ NMR (75 MHz, $\left.\mathrm{CDCl}_{3}\right) \delta 159.5$ (C), 140.2 (C), 128.9 $(\mathrm{CH}), 128.7(\mathrm{CH}), 127.6(\mathrm{CH}), 62.2(\mathrm{CH}), 31.4\left(\mathrm{CH}_{3}\right), 28.1\left(\mathrm{CH}_{3}\right)$; LRMS (CI+) $m / z 255$ $\left(\mathrm{M}+\mathrm{H}^{+}, 100\right)$; HRMS (EI+): calcd for $\mathrm{C}_{16} \mathrm{H}_{19} \mathrm{~N}_{2} \mathrm{O}\left(\mathrm{M}+\mathrm{H}^{+}\right)$255.1492, found: 255.1497 [ $\Delta$ 
1.9 ppm]; EA found: C 75.56\%, H 7.28\%, N 11.31\%. $\mathrm{C}_{16} \mathrm{H}_{18} \mathrm{~N}_{2} \mathrm{O}$ requires $\mathrm{C} 75.56 \%, \mathrm{H}$ $7.13 \%, \mathrm{~N} 11.01 \%$.

\section{( \pm )-1,3-Dimethyl-1-(phenyl(o-tolyl)methyl)urea 3e}

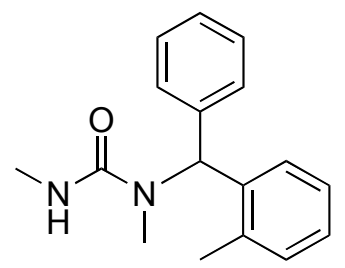

General procedure $3 \mathrm{~b}$ was followed. Flash column chromatography $\left(\mathrm{SiO}_{2} ; 2: 1\right.$ Petrol:EtOAc) gave the title compound as a white solid (151 mg, 76\%). m.p. $109-110^{\circ} \mathrm{C}$; Rf 0.15 (Petrol:EtOAc, 2:1); IR $v_{\max }\left(\mathrm{CHCl}_{3}\right) / \mathrm{cm}^{-1} 3346(\mathrm{~N}-\mathrm{H}), 1629(\mathrm{C}=\mathrm{O}) ;{ }^{1} \mathbf{H}$ NMR $\left(300 \mathrm{MHz}, \mathrm{CDCl}_{3}\right) \delta$ 7.41-7.31 (m, 3H), 7.26-7.14 (m, 5H), 6.97 (brd, $J=8 \mathrm{~Hz}, 1 \mathrm{H}$ ),

6.65 (s, 1H), 4.42 (brs, 1H), 2.87 (brs, 3H), 2.73 (s, 3H), 2.28 (s, 3H); ${ }^{13}$ C NMR (75 $\left.\mathrm{MHz}, \mathrm{CDCl}_{3}\right) \delta 159.3(\mathrm{C}), 140.3(\mathrm{C}), 138.9(\mathrm{C}), 137.7(\mathrm{C}), 131.0(\mathrm{CH}), 128.9(\mathrm{CH})$, $128.7(\mathrm{CH}), 128.4(\mathrm{CH}), 127.9(\mathrm{CH}), 127.6(\mathrm{CH}), 126.2(\mathrm{CH}), 60.5(\mathrm{CH}), 31.8\left(\mathrm{CH}_{3}\right)$, $28.1\left(\mathrm{CH}_{3}\right), 19.7\left(\mathrm{CH}_{3}\right)$; LRMS $(\mathrm{CI}+) \mathrm{m} / z 269\left(\mathrm{M}+\mathrm{H}^{+}, 100\right)$; HRMS (EI+): calcd for $\mathrm{C}_{17} \mathrm{H}_{21} \mathrm{~N}_{2} \mathrm{O}\left(\mathrm{M}+\mathrm{H}^{+}\right)$269.1648, found: 269.1652 [ $\Delta 1.4 \mathrm{ppm}$ ].

( \pm )-1-((2-Methoxyphenyl)(phenyl)methyl)-1,3-dimethylurea 3f<smiles>CNC(=O)N(C)C(c1ccccc1)c1ccccc1OC</smiles>

General procedure $3 \mathrm{~b}$ was followed. Flash column chromatography $\left(\mathrm{SiO}_{2} ; 1: 1\right.$ Petrol:EtOAc) gave the title compound as a white solid (152 mg, 75\%). m.p. $109-112^{\circ} \mathrm{C}$; Rf 0.15 (Petrol:EtOAc, 2:1); IR $v_{\max }\left(\mathrm{CHCl}_{3}\right) / \mathrm{cm}^{-1} 3357(\mathrm{~N}-\mathrm{H}), 1633(\mathrm{C}=\mathrm{O}) ;{ }^{1} \mathbf{H}$ NMR $\left(300 \mathrm{MHz}, \mathrm{CDCl}_{3}\right) \delta$ 7.39-7.26 (m, 4H), 7.20-7.15 (m, 2H), 7.04-6.92 (m, 3H), $6.64(\mathrm{~s}$, 1H), 4.42 (brd, $J=4 \mathrm{~Hz}, 1 \mathrm{H}), 3.81(\mathrm{~s}, 3 \mathrm{H}), 2.87(\mathrm{~d}, J=4 \mathrm{~Hz}, 3 \mathrm{H}), 2.74(\mathrm{~s}, 3 \mathrm{H}) ;{ }^{13} \mathrm{C}$ NMR $\left(75 \mathrm{MHz}, \mathrm{CDCl}_{3}\right) \delta 159.7(\mathrm{C}), 157.7(\mathrm{C}), 140.3(\mathrm{C}), 129.7(\mathrm{CH}), 129.2(\mathrm{CH})$, $128.7(\mathrm{CH}), 128.5(\mathrm{CH}), 128.3(\mathrm{CH}), 127.4(\mathrm{CH}), 120.7(\mathrm{CH}), 110.9(\mathrm{CH}), 57.9(\mathrm{CH})$, $55.8\left(\mathrm{CH}_{3}\right), 31.9\left(\mathrm{CH}_{3}\right), 28.1\left(\mathrm{CH}_{3}\right) ; \quad$ LRMS $(\mathrm{CI}+) \mathrm{m} / z 285\left(\mathrm{M}+\mathrm{H}^{+}, 100\right), 197$ 
$\left(\left[\mathrm{C}_{6} \mathrm{H}_{4}(\mathrm{OMe}) \mathrm{CHPh}\right]^{+}, \quad 35\right), 89 \quad\left(\mathrm{CO}(\mathrm{NHMe})_{2}+\mathrm{H}^{+}, 35\right)$; HRMS (EI+): calcd for $\mathrm{C}_{17} \mathrm{H}_{21} \mathrm{~N}_{2} \mathrm{O}_{2}\left(\mathrm{M}+\mathrm{H}^{+}\right) 285.1598$ found: 285.1603 [ $\Delta-1.7$ ppm]. EA found: $\mathrm{C} 72.04 \%, \mathrm{H}$ 7.46\%, N 9.83\%. $\mathrm{C}_{17} \mathrm{H}_{20} \mathrm{~N}_{2} \mathrm{O}_{2}$ requires C 71.81\%, H 7.09\%, N 9.85\%.

( \pm )-1-((2,6-Dimethylphenyl)(phenyl)methyl)-1,3-dimethylurea 3g

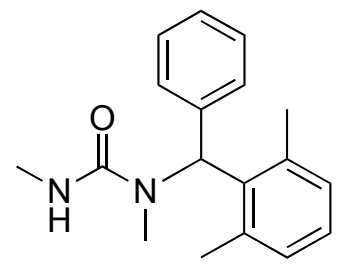

General procedure $3 \mathrm{~b}$ was followed. Flash column chromatography $\left(\mathrm{SiO}_{2} ; 21\right.$ Petrol:EtOAc) gave the title compound as a white solid (163 mg, 82\%). m.p. $174-175{ }^{\circ} \mathrm{C}$; Rf 0.10 (Petrol:EtOAc, 2:1); IR v $v_{\max }\left(\mathrm{CHCl}_{3}\right) / \mathrm{cm}^{-1} 3353(\mathrm{~N}-\mathrm{H}), 1628(\mathrm{C}=\mathrm{O}) ;{ }^{1} \mathbf{H}$ NMR (300 MHz, $\left.\mathrm{CDCl}_{3}\right) \delta$ 7.22-7.01 (m, 5H), 7.39-7.22 (m, 3H), 6.69 (s, 1H), 4.53 (brs, $1 \mathrm{H}$ ), $2.85(\mathrm{~d}, J=4 \mathrm{~Hz}, 3 \mathrm{H}), 2.77$ (s, 3H), $2.10(\mathrm{~s}, 6 \mathrm{H}) ;{ }^{13} \mathbf{C} \mathbf{N M R}\left(75 \mathrm{MHz}, \mathrm{CDCl}_{3}\right) \delta 159.6$ (C), $141.4(\mathrm{C}), 138.6(\mathrm{C}), 136.3(\mathrm{C}), 130.0(\mathrm{CH}), 129.1(\mathrm{CH}), 128.0(\mathrm{CH}), 127.2(\mathrm{CH})$, $127.1(\mathrm{CH}), 60.4(\mathrm{CH}), 31.3\left(\mathrm{CH}_{3}\right), 28.2\left(\mathrm{CH}_{3}\right), 21.9\left(\mathrm{CH}_{3}\right) ;$ LRMS $(\mathrm{CI}+) \mathrm{m} / z 383$ $\left(\mathrm{M}+\mathrm{H}^{+}, 100\right), 195\left(\left[\mathrm{C}_{6} \mathrm{H}_{3}(\mathrm{Me})_{2} \mathrm{CHPh}\right]^{+}, 20\right), 89\left(\mathrm{CO}(\mathrm{NHMe})_{2}+\mathrm{H}^{+}, 30\right)$; HRMS (EI+): calcd for $\mathrm{C}_{18} \mathrm{H}_{23} \mathrm{~N}_{2} \mathrm{O}\left(\mathrm{M}+\mathrm{H}^{+}\right)$283.1805, found: 283.1810 [ $\left.\Delta 1.7 \mathrm{ppm}\right]$.

\section{(土)-1-((4-Chlorophenyl)(phenyl)methyl)-1,3-dimethylurea 3h}

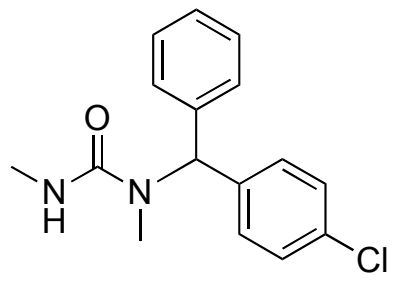

General procedure $3 \mathrm{~b}$ was followed. Flash column chromatography $\left(\mathrm{SiO}_{2} ; 1: 1\right.$ Petrol:EtOAc) gave the title compound as a white solid (138 mg, 69\%). m.p. $96-98{ }^{\circ} \mathrm{C}$; Rf 0.15 (Petrol:EtOAc, 1:1); IR $\boldsymbol{v}_{\max }\left(\mathrm{CHCl}_{3}\right) / \mathrm{cm}^{-1} 3351(\mathrm{~N}-\mathrm{H}), 1631(\mathrm{C}=\mathrm{O}) ;{ }^{1} \mathbf{H}$ NMR (300 MHz, $\mathrm{CDCl}_{3}$ ) $\delta$ 7.37-7.25 (m, 5H), 7.21-7.09 (m, 4H), 6.64 (s, 1H), 5.10-4.10 (brs, 1H), 2.84 (s, 3H), 2.66 (s, 3H); ${ }^{13} \mathbf{C}$ NMR (75 MHz, CDCl $) \delta 158.9$ (C), 139.3 (C), 138.4 (C), $132.9(\mathrm{C}), 129.8(\mathrm{CH}), 128.4(\mathrm{CH}), 128.4(\mathrm{CH}), 127.4(\mathrm{CH}), 61.1(\mathrm{CH}), 30.8\left(\mathrm{CH}_{3}\right)$, 
$27.7\left(\mathrm{CH}_{3}\right)$ [two $\mathrm{CH}$ peaks overlap]; LRMS $(\mathrm{CI}+) \mathrm{m} / \mathrm{z} 289\left(\mathrm{M}+\mathrm{H}^{+}, 100\right), 201$ $\left(\left[\mathrm{C}_{6} \mathrm{H}_{4}(\mathrm{Cl}) \mathrm{CHPh}\right]^{+}, 5\right)$; HRMS (EI+): calcd for $\mathrm{C}_{16} \mathrm{H}_{18} \mathrm{~N}_{2} \mathrm{OCl}\left(\mathrm{M}+\mathrm{H}^{+}\right)$289.1102, found: $289.1106[\Delta 1.3 \mathrm{ppm}]$.

( \pm )-1-((4-Methoxyphenyl)(phenyl)methyl)-1,3-dimethylurea 3i

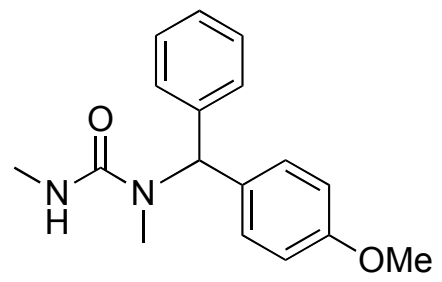

General procedure $3 \mathrm{~b}$ was followed. Flash column chromatography $\left(\mathrm{SiO}_{2} ; 2: 1\right.$ Petrol:EtOAc) gave the title compound as a white solid (152 mg, 76\%). m.p. $100-102{ }^{\circ} \mathrm{C}$; Rf 0.74 (Petrol:EtOAc, 1:1); IR $v_{\max }\left(\mathrm{CH}_{2} \mathrm{Cl}_{2}\right) / \mathrm{cm}^{-1} 3350(\mathrm{~N}-\mathrm{H}), 1628(\mathrm{C}=\mathrm{O}) ;{ }^{1} \mathbf{H}$ NMR $\left(300 \mathrm{MHz}, \mathrm{CDCl}_{3}\right) \delta$ 7.42-7.20 (m, 5H), 7.19-7.12 (m, 2H), 6.96-6.88 (m, 2H), 6.73 (s, 1H), 4.57 (brs, 1H), 3.85 (s, 3H), 2.88 (s, 3H), 2.73 (s, 3H); ${ }^{13} \mathbf{C}$ NMR $\left(75 \mathrm{MHz}, \mathrm{CDCl}_{3}\right)$ \& $159.5(\mathrm{C}), 159.1(\mathrm{C}), 140.4(\mathrm{C}), 132.2(\mathrm{C}), 130.2(\mathrm{CH}), 128.7(\mathrm{CH}), 128.6(\mathrm{CH}), 127.5$ $(\mathrm{CH}), 114.1(\mathrm{CH}), 61.8(\mathrm{CH}), 55.6\left(\mathrm{CH}_{3}\right), 31.3\left(\mathrm{CH}_{3}\right), 28.1\left(\mathrm{CH}_{3}\right)$; LRMS (CI+) $\mathrm{m} / z 285$ $\left(\mathrm{M}+\mathrm{H}^{+}, 100\right)$; HRMS $(\mathrm{EI}+)$ calcd for $\mathrm{C}_{17} \mathrm{H}_{21} \mathrm{~N}_{2} \mathrm{O}_{2}\left(\mathrm{M}+\mathrm{H}^{+}\right)$: 285.1598 , found: 285.1599 $[\Delta 0.3 \mathrm{ppm}]$.

\section{N-Methyldiphenylmethanamine 4d}

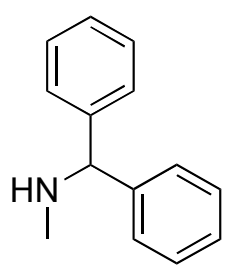

To a solution of $\mathbf{3 d}$ (125 $\mathrm{mg}, 0.49 \mathrm{mmol})$ in anhydrous toluene ( $3 \mathrm{ml})$ was slowly added a solution of DIBAL (1.0 M solution in toluene, $4.9 \mathrm{ml}, 4.49 \mathrm{mmol})$. The reaction mixture was heated at reflux for 48 hours. The reaction mixture was cooled to $0{ }^{\circ} \mathrm{C}$ and quenched by successive addition of water $(0.18 \mathrm{ml}), 3.75 \mathrm{M}$ aqueous $\mathrm{NaOH}$ solution $(0.18 \mathrm{ml})$ and water $(0.49 \mathrm{ml}){ }^{4}$ The mixture allowed to warm to room temperature and stirred for 15 minutes. $\mathrm{MgSO}_{4}$ was added, the mixture was stirred for 15 minutes and filtered. The filtrate was concentrated under reduced pressure to give the a crude mixture which was 
purified by flash column chromatography $\left(\mathrm{SiO}_{2} ; 4: 1\right.$ Petrol:EtOAc) to yield the title compound as a white solid (81 mg, 84\%). m.p. $39-40{ }^{\circ} \mathrm{C}$ (Lit. $\left.{ }^{5} 38-39{ }^{\circ} \mathrm{C}\right) ;{ }^{1} \mathbf{H}$ NMR (300 $\left.\mathrm{MHz}, \mathrm{CDCl}_{3}\right) \delta$ 7.55-7.52 (m, 10H), $4.77(\mathrm{~s}, 1 \mathrm{H}), 2.47$ (s, 3H), 2.14 (brs, 1H). All data were consistent with those previously reported. ${ }^{5,6}$

\section{( \pm )-N-Methyl-1-phenyl-1-o-tolylmethanamine 4e}

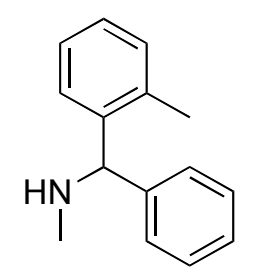

General procedure 4 was followed. Flash column chromatography $\left(\mathrm{SiO}_{2} ; 2: 1\right.$ Petrol:Et $\left.{ }_{2} \mathrm{O}\right)$ gave ( $( \pm)$-1,3-Dimethyl-3-nitroso-1-(phenyl(o-tolyl)methyl)urea as a pale yellow oil (56 mg, 90\%). Rf $0.58\left(\mathrm{Et}_{2} \mathrm{O}-\mathrm{Petrol}, 1: 1\right)$; IR $\boldsymbol{V}_{\max }($ neat $) / \mathrm{cm}^{-1} 1688(\mathrm{C}=\mathrm{O}) ;{ }^{1} \mathbf{H}$ NMR $\left(500 \mathrm{MHz}, \mathrm{CDCl}_{3}\right) \delta$ 7.30-7.27 (m, 2H), 7.24-7.21 (m, 1H), 7.16-7.12 (m, 4H), 7.07 (m, 1H), 6.93 (d, $J=7.7 \mathrm{~Hz}, 1 \mathrm{H}), 6.74$ (brs, 1H), 3.08 (s, 3H), 2.82 (s, 3H), 2.19 (s, 3H); ${ }^{13}$ C NMR (125 MHz, $\left.\mathrm{CDCl}_{3}\right) \delta 156.1$ (C), 138.2 (C), 137.5 (C), 136.9 (C), 130.8 $(\mathrm{CH}), 128.6(\mathrm{CH}), 128.6(\mathrm{CH}), 128.0(\mathrm{CH}), 127.8(\mathrm{CH}), 127.5(\mathrm{CH}), 125.9(\mathrm{CH}), 62.5$ $(\mathrm{CH}), 35.6\left(\mathrm{CH}_{3}\right), 29.6\left(\mathrm{CH}_{3}\right), 19.4\left(\mathrm{CH}_{3}\right)$; LRMS (ES+): $\mathrm{m} / z 181(100), 320\left(\mathrm{M}+\mathrm{Na}^{+}\right.$, 70); HRMS (EI+): calcd for $\mathrm{C}_{17} \mathrm{H}_{19} \mathrm{O}_{2} \mathrm{~N}_{3} \mathrm{Na}_{1}\left(\mathrm{M}+\mathrm{Na}^{+}\right)$: 320.1369, found: $320.1376[\Delta 2.0$ ppm].

General procedure 5 was followed. Flash column chromatography $\left(\mathrm{SiO}_{2} ; 1: 1\right.$ Petrol:EtOAc) gave the title compound as a pale yellow oil (42 mg, 80\%). Rf 0.29 (EtOAc-Petrol, 2:1); IR $v_{\max }\left(\right.$ neat) $/ \mathrm{cm}^{-1} 1490 ;{ }^{1} \mathbf{H}$ NMR (500 MHz, $\left.\mathrm{CDCl}_{3}\right) \delta 7.49(\mathrm{~d}, J$ = 7.7 Hz, 1H), 7.24-7.18 (m, 4H), 7.16-7.10 (m, 2H), 7.07-7.01 (m, 2H), $4.79(\mathrm{~s}, 1 \mathrm{H})$, 2.34 (s, 3H), 2.20 (s, 3H); ${ }^{13}$ C NMR (125 MHz, $\left.\mathrm{CDCl}_{3}\right) \delta 142.9$ (C), 141.3 (C), 135.7 (C), $130.4(\mathrm{CH}), 128.3(\mathrm{CH}), 127.9(\mathrm{CH}), 126.8(\mathrm{CH}), 126.6(\mathrm{CH}), 126.3(\mathrm{CH}), 126.1$ $(\mathrm{CH}), 65.2(\mathrm{CH}), 35.2\left(\mathrm{CH}_{3}\right), 19.5\left(\mathrm{CH}_{3}\right)$. LRMS (ES+): $\mathrm{m} / z 212\left(\mathrm{M}+\mathrm{H}^{+}, 32\right), 181$ (100); HRMS (EI+): calcd for $\mathrm{C}_{15} \mathrm{H}_{18} \mathrm{~N}_{1}\left(\mathrm{M}+\mathrm{H}^{+}\right)$: 212.1434, found: 212.1425 [ $\left.\Delta-4.1 \mathrm{ppm}\right]{ }^{7}$ 
( \pm )-1-(2-Methoxyphenyl)- $N$-methyl-1-phenylmethanamine 4f<smiles>CNC(c1ccccc1)c1ccccc1OC</smiles>

General procedure 4 was followed. Flash column chromatography $\left(\mathrm{SiO}_{2} ; 1: 1\right.$

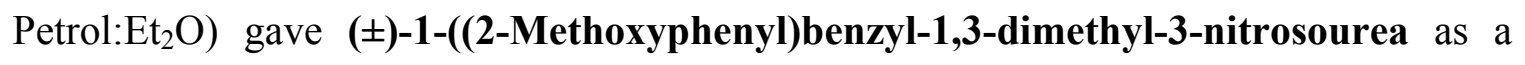
pale yellow oil (122 mg, 96\%). Rf $0.32\left(\mathrm{Et}_{2} \mathrm{O}-\mathrm{Petrol}, 1: 1\right)$; IR $\boldsymbol{V}_{\max }($ neat $) / \mathrm{cm}^{-1} 1690$ $(\mathrm{C}=\mathrm{O}) ;{ }^{1} \mathbf{H}$ NMR $\left(500 \mathrm{MHz}, \mathrm{CDCl}_{3}\right) \delta$ 7.28-7.18 (m, 4H), $7.14(\mathrm{~d}, J=7.8 \mathrm{~Hz}, 2 \mathrm{H}), 6.88-$ $6.79(\mathrm{~m}, 3 \mathrm{H}), 6.70$ (brs, $1 \mathrm{H}), 3.70$ (s, 3H), 3.12 (s, 3H), 2.77 (s, 3H); ${ }^{13} \mathbf{C}$ NMR (125 $\left.\mathrm{MHz}, \mathrm{CDCl}_{3}\right) \delta 157.7(\mathrm{C}), 156.8(\mathrm{C}), 137.9(\mathrm{C}), 130.0(\mathrm{CH}), 129.4(\mathrm{CH}), 128.3(\mathrm{CH})$, $127.5(\mathrm{CH}), 127.1(\mathrm{CH}), 126.7(\mathrm{C}), 120.1(\mathrm{CH}), 110.4(\mathrm{CH}), 60.6(\mathrm{CH}), 55.5\left(\mathrm{CH}_{3}\right), 34.3$ $\left(\mathrm{CH}_{3}\right), 29.7\left(\mathrm{CH}_{3}\right)$; LRMS (ES+): m/z 197 (100), $336\left(\mathrm{M}+\mathrm{Na}^{+}, 37\right)$; HRMS (EI+): calcd for $\mathrm{C}_{17} \mathrm{H}_{19} \mathrm{O}_{3} \mathrm{~N}_{3} \mathrm{Na}_{1}\left(\mathrm{M}+\mathrm{Na}^{+}\right)$: 336.1319, found: 336.1327 [ $2.5 \mathrm{ppm}$ ].

General procedure 5 was followed. Flash column chromatography $\left(\mathrm{SiO}_{2} ; 1: 2\right.$ Petrol:EtOAc) gave the title compound as a pale yellow oil (71 mg, 86\%). Rf 0.33 (EtOAc-Petrol, 2:1); IR $v_{\max }($ neat $) / \mathrm{cm}^{-1} 1489 ;{ }^{1} \mathbf{H}$ NMR $\left(500 \mathrm{MHz}, \mathrm{CDCl}_{3}\right) \delta$ 7.32-7.30 $(\mathrm{m}, 3 \mathrm{H}), 7.18(\mathrm{t}, J=7.6 \mathrm{~Hz}, 2 \mathrm{H}), 7.11-7.07(\mathrm{~m}, 2 \mathrm{H}), 6.84(\mathrm{t}, J=7.0 \mathrm{~Hz}, 1 \mathrm{H}), 6.73(\mathrm{~d}, J$ $=8.1 \mathrm{~Hz}, 1 \mathrm{H}), 4.99(\mathrm{~s}, 1 \mathrm{H}), 3.67(\mathrm{~s}, 3 \mathrm{H}), 2.31(\mathrm{~s}, 3 \mathrm{H}) ;{ }^{13} \mathbf{C} \mathbf{N M R}\left(125 \mathrm{MHz}, \mathrm{CDCl}_{3}\right) \delta$ $156.8(\mathrm{C}), 143.4(\mathrm{C}), 131.9(\mathrm{C}), 128.0(\mathrm{CH}), 127.7(\mathrm{CH}), 127.5(\mathrm{CH}), 127.5(\mathrm{CH}), 126.5$ $(\mathrm{CH}), 120.6(\mathrm{CH}), 110.5(\mathrm{CH}), 62.4(\mathrm{CH}), 55.2\left(\mathrm{CH}_{3}\right), 35.0\left(\mathrm{CH}_{3}\right)$. LRMS (ES+): $\mathrm{m} / z$ $228\left(\mathrm{M}+\mathrm{H}^{+}, 62\right), 197(100)$; HRMS $(\mathrm{EI}+)$ : calcd for $\mathrm{C}_{15} \mathrm{H}_{18} \mathrm{O}_{1} \mathrm{~N}_{1}\left(\mathrm{M}+\mathrm{H}^{+}\right)$: 228.1383, found: $228.1391[\Delta 3.5 \mathrm{ppm}]$.

( \pm )-1-(2,6-Dimethylphenyl)- $N$-methyl-1-phenylmethanamine 4g<smiles>CNC(c1ccccc1)c1c(C)cccc1C</smiles>

General procedure 4 was followed. Flash column chromatography $\left(\mathrm{SiO}_{2} ; 2: 1\right.$

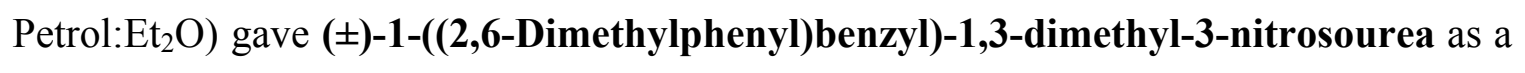


pale yellow oil (44 mg, 98\%). Rf 0.65 (Et ${ }_{2} \mathrm{O}-$ Petrol, 1:1); IR $v_{\max }($ neat $) / \mathrm{cm}^{-1} 1688$ $(\mathrm{C}=\mathrm{O}) ;{ }^{1} \mathbf{H}$ NMR $\left(500 \mathrm{MHz}, \mathrm{CDCl}_{3}\right) \delta 7.26(\mathrm{t}, J=7.5 \mathrm{~Hz}, 2 \mathrm{H}), 7.18(\mathrm{t}, J=7.4 \mathrm{~Hz}, 1 \mathrm{H})$, 7.12-7.06 (m, 3H), 7.04 (brs, 1H), 6.97 (d, $J=7.6 \mathrm{~Hz}, 2 \mathrm{H}), 3.08$ (s, 3H), 2.93 (s, 3H), 2.03 (s, 6H); ${ }^{13} \mathrm{C}$ NMR (125 MHz, $\left.\mathrm{CDCl}_{3}\right) \delta 156.0$ (C), 139.7 (C), 138.8 (C), 134.4 (C), $129.8(\mathrm{CH}), 128.8(\mathrm{CH}), 128.2(\mathrm{CH}), 126.9(\mathrm{CH}), 126.2(\mathrm{CH}), 61.7(\mathrm{CH}), 34.7\left(\mathrm{CH}_{3}\right)$, $29.7\left(\mathrm{CH}_{3}\right), 21.7\left(\mathrm{CH}_{3}\right)$; LRMS (ES+): m/z 195 (100), $283(26), 312\left(\mathrm{M}+\mathrm{H}^{+}, 53\right), 329$ $\left(\mathrm{M}+\mathrm{NH}_{4}{ }^{+}, 32\right)$; HRMS (EI+): calcd for $\mathrm{C}_{18} \mathrm{H}_{25} \mathrm{O}_{2} \mathrm{~N}_{4}\left(\mathrm{M}+\mathrm{NH}_{4}{ }^{+}\right)$: 329.1972, found: $329.1962[\Delta-3.0 \mathrm{ppm}]$.

General procedure 5 was followed. Flash column chromatography $\left(\mathrm{SiO}_{2} ; 1: 2\right.$ Petrol:EtOAc) gave the title compound as a pale yellow oil (26 $\mathrm{mg}, 86 \%)$. Rf 0.36 (EtOAc-Petrol, 2:1); IR $v_{\max }$ (neat) $/ \mathrm{cm}^{-1} 1473 ;{ }^{1} \mathbf{H}$ NMR $\left(500 \mathrm{MHz}, \mathrm{CDCl}_{3}\right) \delta$ 7.22-7.16 (m, 4H), 7.11-7.08 (m, 1H), 7.01-6.98 (m, 1H), $6.92(\mathrm{~d}, J=7.5 \mathrm{~Hz}, 2 \mathrm{H}), 5.26-5.24$ (m, 1H), 2.39 (s, 3H), 2.18 (s, 6H); ${ }^{13} \mathbf{C}$ NMR (125 MHz, $\left.\mathrm{CDCl}_{3}\right) \delta 143.2(\mathrm{C}), 139.6(\mathrm{C})$, $137.1(\mathrm{C}), 129.2(\mathrm{CH}), 128.0(\mathrm{CH}), 126.8(\mathrm{CH}), 126.4(\mathrm{CH}), 126.0(\mathrm{CH}), 62.4(\mathrm{CH}), 35.0$ $\left(\mathrm{CH}_{3}\right), 21.0\left(\mathrm{CH}_{3}\right)$. LRMS (ES+): m/z $226\left(\mathrm{M}+\mathrm{H}^{+}, 42\right), 195$ (100); HRMS (EI+): calcd for $\mathrm{C}_{16} \mathrm{H}_{20} \mathrm{~N}_{1}\left(\mathrm{M}+\mathrm{H}^{+}\right): 226.1590$, found: 226.1593 [ $\left.1.2 \mathrm{ppm}\right]$.

\section{( \pm )-1,3-Dimethyl-1-phenyl-3-(1-phenylethyl)urea 5a}

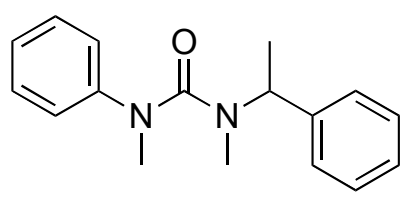

General procedures 1 and $2 \mathrm{~b}$ were followed. Flash column chromatography $\left(\mathrm{SiO}_{2} ; 2: 1\right.$ Petrol:EtOAc) gave the title compound as a pale yellow oil (1.6 g, 75\%). Rf 0.37 (Petrol:EtOAc, 2:1); IR $v_{\max }\left(\mathrm{CHCl}_{3}\right) / \mathrm{cm}^{-1} 1643(\mathrm{C}=\mathrm{O}) ;{ }^{1} \mathbf{H}$ NMR $\left(300 \mathrm{MHz}, \mathrm{CDCl}_{3}\right) \delta$ 7.40-7.26 (m, 7H), 7.19-7.12 (m, 3H), 5.62 (q, $J=7$ Hz, 1H), 3.31 (s, 3H), $2.26(\mathrm{~s}, 3 \mathrm{H})$, $1.46(\mathrm{~d}, J=7 \mathrm{~Hz}, 3 \mathrm{H}) ;{ }^{13} \mathbf{C}$ NMR $\left(75 \mathrm{MHz}, \mathrm{CDCl}_{3}\right) \delta 162.4$ (C), 147.3 (C), 141.3 (C), $129.7(\mathrm{CH}), 128.6(\mathrm{CH}), 127.6(\mathrm{CH}), 127.4(\mathrm{CH}), 124.8(\mathrm{CH}), 124.5(\mathrm{CH}), 54.1(\mathrm{CH})$, $40.4\left(\mathrm{CH}_{3}\right), 31.0\left(\mathrm{CH}_{3}\right), 15.8\left(\mathrm{CH}_{3}\right)$; LRMS (CI+) $\mathrm{m} / z 269\left(\mathrm{M}+\mathrm{H}^{+}, 100\right)$; HRMS (EI+) calcd for $\mathrm{C}_{17} \mathrm{H}_{20} \mathrm{~N}_{2} \mathrm{ONa}\left(\mathrm{M}+\mathrm{Na}^{+}\right): 291.1468$, found: 291.1461 [ $\Delta-2.4 \mathrm{ppm}$ ]. 


\section{1-(4-Methoxyphenyl)-1,3-dimethyl-3-((R)-1-phenylethyl)urea 5b}

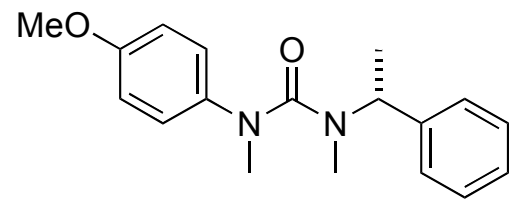

General procedures 1 and $2 \mathrm{~b}$ were followed. Flash column chromatography $\left(\mathrm{SiO}_{2} ; 1: 1\right.$ Petrol:Et $\left.{ }_{2} \mathrm{O}\right)$ gave the title compound as a colourless oil $(805 \mathrm{mg}, 81 \%)$. $\mathbf{R f} 0.32\left(\mathrm{Et}_{2} \mathrm{O}-\right.$ Petrol, 1:1); $[\boldsymbol{\alpha}]_{\mathbf{D}}{ }^{22}=+25.1\left(c=2.85, \mathrm{CH}_{3} \mathrm{OH}\right) ; \mathbf{I R} \boldsymbol{v}_{\max }\left(\mathrm{CHCl}_{3}\right) / \mathrm{cm}^{-1} 1641(\mathrm{C}=\mathrm{O}) ;{ }^{1} \mathbf{H}$ NMR $\left(500 \mathrm{MHz}, \mathrm{CDCl}_{3}\right) \delta$ 7.30-7.24 (m, 4H), 7.22-7.19 (m, 1H), $7.03(\mathrm{~d}, J=8.9 \mathrm{~Hz}$, 2H), $6.83(\mathrm{~d}, J=8.9 \mathrm{~Hz}, 2 \mathrm{H}), 5.53(\mathrm{q}, J=7.0 \mathrm{~Hz}, 1 \mathrm{H}), 3.76(\mathrm{~s}, 3 \mathrm{H}), 3.18(\mathrm{~s}, 3 \mathrm{H}), 2.17$ (s, 3H), $1.37(\mathrm{~d}, J=7.0 \mathrm{~Hz}, 3 \mathrm{H}) ;{ }^{13} \mathbf{C}$ NMR (125 MHz, $\left.\mathrm{CDCl}_{3}\right) \delta 162.3(\mathrm{C}), 156.6(\mathrm{C})$, $141.0(\mathrm{C}), 139.9(\mathrm{C}), 128.0(\mathrm{CH}), 127.1(\mathrm{CH}), 126.8(\mathrm{CH}), 125.9(\mathrm{CH}), 114.4(\mathrm{CH}), 55.2$ $\left(\mathrm{CH}_{3}\right), 53.6(\mathrm{CH}), 40.6\left(\mathrm{CH}_{3}\right), 30.5\left(\mathrm{CH}_{3}\right), 15.4\left(\mathrm{CH}_{3}\right)$; LRMS (ES+): $m / z 321\left(\mathrm{M}+\mathrm{Na}^{+}\right.$, 100), $299\left(\mathrm{M}+\mathrm{H}^{+}, 20\right)$; HRMS (EI+): calcd for $\mathrm{C}_{18} \mathrm{H}_{23} \mathrm{O}_{2} \mathrm{~N}_{2}\left(\mathrm{M}+\mathrm{H}^{+}\right)$: 299.1754, found: $299.1749[\Delta-1.7 \mathrm{ppm}]$.

( \pm )-1-(2-Methoxyphenyl)-1,3-dimethyl-3-(1-phenylethyl)urea 5c<smiles>COc1ccccc1N(C)C(=O)N(C)C(C)c1ccccc1</smiles>

General procedures 1 and $2 \mathrm{~b}$ were followed. Flash column chromatography $\left(\mathrm{SiO}_{2} ; 1: 1\right.$ Petrol:EtOAc) gave the title compound as a colourless oil (693 mg, 62\%). Rf 0.93 (EtOAc-Petrol, 2:1); IR $v_{\max }\left(\right.$ neat) $/ \mathrm{cm}^{-1} 1641(\mathrm{C}=\mathrm{O}) ;{ }^{1} \mathbf{H}$ NMR $\left(500 \mathrm{MHz}, \mathrm{CDCl}_{3}\right) \delta$ 7.20-7.15 (m, 4H), 7.12-7.07 (m, 2H), $7.00(\mathrm{dd}, J=8.0,1.7 \mathrm{~Hz}, 1 \mathrm{H}), 6.83-6.80(\mathrm{~m}, 2 \mathrm{H})$, $5.41(\mathrm{q}, J=7.0 \mathrm{~Hz}, 1 \mathrm{H}), 3.77(\mathrm{~s}, 3 \mathrm{H}), 3.03(\mathrm{~s}, 3 \mathrm{H}), 2.02(\mathrm{~s}, 3 \mathrm{H}), 1.25(\mathrm{~d}, J=7.0 \mathrm{~Hz}, 3 \mathrm{H})$;

${ }^{13}$ C NMR (125 MHz, $\left.\mathrm{CDCl}_{3}\right) \delta 162.8(\mathrm{C}), 154.1(\mathrm{C}), 141.3(\mathrm{C}), 135.0(\mathrm{C}), 128.1(\mathrm{CH})$, $127.5(\mathrm{CH}), 127.2(\mathrm{CH}), 127.2(\mathrm{CH}), 126.8(\mathrm{CH}), 121.0(\mathrm{CH}), 111.7(\mathrm{CH}), 55.4\left(\mathrm{CH}_{3}\right)$, $53.7(\mathrm{CH}), 38.5\left(\mathrm{CH}_{3}\right), 30.1\left(\mathrm{CH}_{3}\right), 15.5\left(\mathrm{CH}_{3}\right)$; LRMS (ES+): $m / z 321\left(\mathrm{M}+\mathrm{Na}^{+}, 50\right), 299$ $\left(\mathrm{M}+\mathrm{H}^{+}, 100\right)$; HRMS (EI+): calcd for $\mathrm{C}_{18} \mathrm{H}_{23} \mathrm{O}_{2} \mathrm{~N}_{2}\left(\mathrm{M}+\mathrm{H}^{+}\right)$: 299.1754, found: 299.1761 $[\Delta 2.3 \mathrm{ppm}]$. 


\section{1-(3,4-Dimethoxyphenyl)-1,3-dimethyl-3-((S)-1-phenylethyl)urea 5d}<smiles>COc1ccc(N(C)C(=O)N(C)[C@@H](C)c2ccccc2)cc1OC</smiles>

General procedures 1 and $2 b$ were followed. Flash column chromatography $\left(\mathrm{SiO}_{2} ; 1: 2\right.$ then 0:1 Petrol:Et $\left.{ }_{2} \mathrm{O}\right)$ gave the title compound as a colourless oil $(1.18 \mathrm{~g}, 87 \%)$. $\mathbf{R f} 0.50$ $\left(\mathrm{Et}_{2} \mathrm{O}\right) ;[\boldsymbol{\alpha}]_{\mathbf{D}}{ }^{22}=-6.4\left(c=1.06, \mathrm{CH}_{3} \mathrm{OH}\right) ; \mathbf{I R} \boldsymbol{v}_{\max }($ neat $) / \mathrm{cm}^{-1} 1637(\mathrm{C}=\mathrm{O}) ;{ }^{1} \mathbf{H}$ NMR $(500$ $\left.\mathrm{MHz}, \mathrm{CDCl}_{3}\right) \delta 7.22-7.18(\mathrm{~m}, 4 \mathrm{H}), 7.14-7.11(\mathrm{~m}, 1 \mathrm{H}), 6.71(\mathrm{~d}, J=8.5 \mathrm{~Hz}, 1 \mathrm{H}), 6.60(\mathrm{dd}$, $J=8.5,2.5 \mathrm{~Hz}, 1 \mathrm{H}), 6.55(\mathrm{~d}, J=2.5 \mathrm{~Hz}, 1 \mathrm{H}), 5.51(\mathrm{q}, J=7.1 \mathrm{~Hz}, 1 \mathrm{H}), 3.76(\mathrm{~s}, 3 \mathrm{H}), 3.71$ (s, 3H), 3.13 (s, 3H), 2.14 (s, 3H), 1.32 (d, $J=7.1 \mathrm{~Hz}, 3 \mathrm{H}) ;{ }^{13} \mathbf{C} \mathbf{N M R}\left(125 \mathrm{MHz}, \mathrm{CDCl}_{3}\right)$ $\delta 162.2(\mathrm{C}), 149.3(\mathrm{C}), 146.3(\mathrm{C}), 141.1(\mathrm{C}), 140.3(\mathrm{C}), 128.2(\mathrm{CH}), 127.2(\mathrm{CH}), 127.0$ $(\mathrm{CH}), 116.9(\mathrm{CH}), 111.5(\mathrm{CH}), 108.6(\mathrm{CH}), 55.9\left(\mathrm{CH}_{3}\right), 55.8\left(\mathrm{CH}_{3}\right), 53.6(\mathrm{CH}), 40.8$ $\left(\mathrm{CH}_{3}\right), 30.6\left(\mathrm{CH}_{3}\right), 15.6\left(\mathrm{CH}_{3}\right)$; LRMS (ES+): $m / z 351\left(\mathrm{M}+\mathrm{Na}^{+}, 100\right), 329\left(\mathrm{M}+\mathrm{H}^{+}, 20\right)$, 151 (33); HRMS (EI+): calcd for $\mathrm{C}_{19} \mathrm{H}_{25} \mathrm{O}_{3} \mathrm{~N}_{2}\left(\mathrm{M}+\mathrm{H}^{+}\right)$: 329.1860 , found: 329.1860 [ $\Delta$ $0.1 \mathrm{ppm}]$.

\section{( \pm )-1-(2-Fluorophenyl)-1,3-dimethyl-3-(1-phenylethyl)urea 5e}<smiles>CC(c1ccccc1)N(C)C(=O)N(C)c1ccccc1F</smiles>

General procedures 1 and $2 \mathrm{~b}$ were followed. Flash column chromatography $\left(\mathrm{SiO}_{2} ; 1: 1\right.$ Petrol:Et $\left.\mathrm{E}_{2} \mathrm{O}\right)$ gave the title compound as a colourless oil $(847 \mathrm{mg}, 81 \%)$. $\mathbf{R f}^{0.28}\left(\mathrm{Et}_{2} \mathrm{O}-\right.$ Petrol, 1:1); IR $v_{\max }($ neat $) / \mathrm{cm}^{-1} 1647(\mathrm{C}=\mathrm{O}) ;{ }^{1} \mathbf{H}$ NMR $\left(500 \mathrm{MHz}, \mathrm{CDCl}_{3}\right) \delta$ 7.20-7.15 (m, 4H), 7.12-7.09 (m, 1H), 7.06- $7.02(\mathrm{~m}, 2 \mathrm{H}), 7.00-6.96(\mathrm{~m}, 2 \mathrm{H}), 5.39$ (q, $J=7.0 \mathrm{~Hz}$, 1H), $3.08(\mathrm{~s}, 3 \mathrm{H}), 2.06(\mathrm{~s}, 3 \mathrm{H}), 1.28(\mathrm{~d}, J=7.0 \mathrm{~Hz}, 3 \mathrm{H}) ;{ }^{13} \mathbf{C ~ N M R}\left(125 \mathrm{MHz}, \mathrm{CDCl}_{3}\right) \delta$ $162.1(\mathrm{C}), 157.1\left(\mathrm{~d},{ }^{1} J_{\mathrm{CF}}=249 \mathrm{~Hz}, \mathrm{C}\right), 140.9(\mathrm{C}), 134.3\left(\mathrm{~d},{ }^{2} J_{\mathrm{CF}}=11.4 \mathrm{~Hz}, \mathrm{C}\right), 128.2$ $(\mathrm{CH}), 127.6\left(\mathrm{~d},{ }^{4} J_{\mathrm{CF}}=1.3 \mathrm{~Hz}, \mathrm{CH}\right), 127.3(\mathrm{CH}), 127.3\left(\mathrm{~d},{ }^{3} J_{\mathrm{CF}}=7.5 \mathrm{~Hz}, \mathrm{CH}\right), 127.0$ $(\mathrm{CH}), 124.8\left(\mathrm{~d},{ }^{3} J_{\mathrm{CF}}=3.7 \mathrm{~Hz}, \mathrm{CH}\right), 116.8\left(\mathrm{~d},{ }^{2} J_{\mathrm{CF}}=20.0 \mathrm{~Hz}, \mathrm{CH}\right), 53.9(\mathrm{CH}), 39.0$ $\left(\mathrm{CH}_{3}\right), 30.3\left(\mathrm{CH}_{3}\right), 15.4\left(\mathrm{CH}_{3}\right)$; LRMS (ES+): m/z $309\left(\mathrm{M}+\mathrm{Na}^{+}, 100\right), 287\left(\mathrm{M}+\mathrm{H}^{+}, 12\right)$; 
HRMS (EI+): calcd for $\mathrm{C}_{17} \mathrm{H}_{20} \mathrm{O}_{1} \mathrm{~N}_{2} \mathrm{~F}_{1}\left(\mathrm{M}+\mathrm{H}^{+}\right)$: 287.1554, found: $287.1547[\Delta-2.5$ ppm].

( \pm )-1-(3-Fluorophenyl)-1,3-dimethyl-3-(1-phenylethyl)urea $5 f$<smiles>CC(c1ccccc1)N(C)C(=O)N(C)c1cccc(F)c1</smiles>

General procedures 1 and $2 b$ were followed. Flash column chromatography $\left(\mathrm{SiO}_{2} ; 1: 1\right.$ Petrol:Et ${ }_{2} \mathrm{O}$ ) gave the title compound as a colourless oil ( $\left.733 \mathrm{mg}, 83 \%\right)$. Rf $0.32\left(\mathrm{Et}_{2} \mathrm{O}-\right.$ Petrol, 1:1); IR $v_{\max }($ neat $) / \mathrm{cm}^{-1} 1650(\mathrm{C}=\mathrm{O}) ;{ }^{1} \mathbf{H}$ NMR $\left(500 \mathrm{MHz}, \mathrm{CDCl}_{3}\right) \delta$ 7.32-7.27 (m, 4H), 7.24-7.20 (m, 2H), 6.84 (dd, $J=7.7,1.7 \mathrm{~Hz}, 1 \mathrm{H}), 6.80-6.73(\mathrm{~m}, 2 \mathrm{H}), 5.56$ (q, $J$ $=7.1 \mathrm{~Hz}, 1 \mathrm{H}), 3.23(\mathrm{~s}, 3 \mathrm{H}), 2.26(\mathrm{~s}, 3 \mathrm{H}), 1.44(\mathrm{~d}, J=7.1 \mathrm{~Hz}, 3 \mathrm{H}) ;{ }^{13} \mathbf{C}$ NMR $(125 \mathrm{MHz}$, $\left.\mathrm{CDCl}_{3}\right) \delta 163.2\left(\mathrm{~d},{ }^{1} J_{\mathrm{CF}}=246.4 \mathrm{~Hz}, \mathrm{C}\right), 161.5(\mathrm{C}), 148.4\left(\mathrm{~d},{ }^{3} J_{\mathrm{CF}}=9.8 \mathrm{~Hz}, \mathrm{C}\right), 140.7$ (C), $130.4\left(\mathrm{~d},{ }^{3} J_{\mathrm{CF}}=9.4 \mathrm{~Hz}, \mathrm{CH}\right), 128.3(\mathrm{CH}), 127.2(\mathrm{CH}), 127.2(\mathrm{CH}), 118.8\left(\mathrm{~d},{ }^{4} J_{\mathrm{CF}}=2.8\right.$ $\mathrm{Hz}, \mathrm{CH}), 110.9\left(\mathrm{~d},{ }^{2} J_{\mathrm{CF}}=21.2 \mathrm{~Hz}, \mathrm{CH}\right), 110.5\left(\mathrm{~d},{ }^{2} J_{\mathrm{CF}}=23.1 \mathrm{~Hz}, \mathrm{CH}\right), 53.8(\mathrm{CH}), 39.5$ $\left(\mathrm{CH}_{3}\right), 30.6\left(\mathrm{CH}_{3}\right), 15.5\left(\mathrm{CH}_{3}\right)$; LRMS (ES+): $m / z 309\left(\mathrm{M}+\mathrm{Na}^{+}, 100\right), 287\left(\mathrm{M}+\mathrm{H}^{+}, 30\right)$; HRMS (EI+): calcd for $\mathrm{C}_{17} \mathrm{H}_{20} \mathrm{O}_{1} \mathrm{~N}_{2} \mathrm{~F}_{1}\left(\mathrm{M}+\mathrm{H}^{+}\right)$: 287.1554, found: 287.1557 [ $\Delta 1.0 \mathrm{ppm}$ ].

\section{1-(4-Fluorophenyl)-1,3-dimethyl-3-((S)-1-phenylethyl)urea 5g}

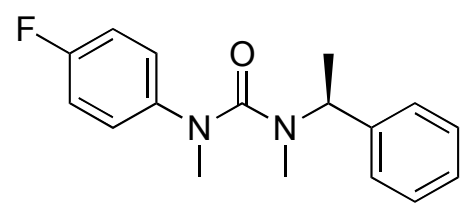

General procedures 1 and $2 \mathrm{~b}$ were followed. Flash column chromatography $\left(\mathrm{SiO}_{2} ; 1: 1\right.$ Petrol: $\left.\mathrm{Et}_{2} \mathrm{O}\right)$ gave the title compound as a colourless oil $(800 \mathrm{mg}, 90 \%)$. $\mathbf{R f} 0.32\left(\mathrm{Et}_{2} \mathrm{O}-\right.$ Petrol, 1:1)

$[\boldsymbol{\alpha}]_{\mathbf{D}}{ }^{21}=-38.6\left(c=2.01, \mathrm{CH}_{3} \mathrm{OH}\right) ; \mathbf{I R} \boldsymbol{v}_{\max }($ neat $) / \mathrm{cm}^{-1} 1646(\mathrm{C}=\mathrm{O}) ;{ }^{1} \mathbf{H} \mathbf{~ N M R}(500 \mathrm{MHz}$, $\left.\mathrm{CDCl}_{3}\right) \delta$ 7.21-7.15 (m, 4H), 7.13-7.10 (m, 1H), 6.98-6.94 (m, 2H), 6.92-6.85 (m, 2H), $5.43(\mathrm{q}, J=7.0 \mathrm{~Hz}, 1 \mathrm{H}), 3.10$ (s, 3H), $2.10(\mathrm{~s}, 3 \mathrm{H}), 1.29$ (d, $J=7.0 \mathrm{~Hz}, 3 \mathrm{H}) ;{ }^{13} \mathbf{C}$ NMR $\left(125 \mathrm{MHz}, \mathrm{CDCl}_{3}\right) \delta 162.1(\mathrm{C}), 159.6\left(\mathrm{~d},{ }^{1} J_{\mathrm{CF}}=244.5 \mathrm{~Hz}, \mathrm{C}\right), 143.1\left(\mathrm{~d},{ }^{4} J_{\mathrm{CF}}=3.3 \mathrm{~Hz}\right.$, C), $140.9(\mathrm{C}), 128.3(\mathrm{CH}), 127.2(\mathrm{CH}), 127.1(\mathrm{CH}), 126.1\left(\mathrm{~d},{ }^{3} J_{\mathrm{CF}}=8.3 \mathrm{~Hz}, \mathrm{CH}\right), 116.2$ 
$\left(\mathrm{d},{ }^{2} J_{\mathrm{CF}}=22.5 \mathrm{~Hz}, \mathrm{CH}\right), 53.8(\mathrm{CH}), 40.4\left(\mathrm{CH}_{3}\right), 30.7\left(\mathrm{CH}_{3}\right), 15.5\left(\mathrm{CH}_{3}\right)$; LRMS (ES+):

$m / z 309\left(\mathrm{M}+\mathrm{Na}^{+}, 100\right), 287\left(\mathrm{M}+\mathrm{H}^{+}, 60\right)$; HRMS $(\mathrm{EI}+)$ : calcd for $\mathrm{C}_{17} \mathrm{H}_{20} \mathrm{O}_{1} \mathrm{~N}_{2} \mathrm{~F}_{1}\left(\mathrm{M}+\mathrm{H}^{+}\right)$: 287.1554, found: $287.1562[\Delta 2.7 \mathrm{ppm}]$.

\section{1-(4-Chlorophenyl)-1,3-dimethyl-3-((S)-1-phenylethyl)urea 5h}

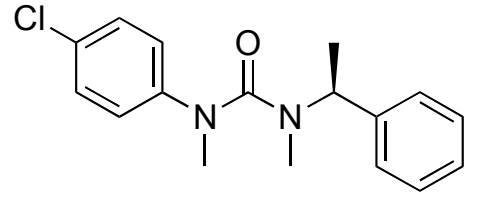

General procedures 1 and $2 b$ were followed. Flash column chromatography $\left(\mathrm{SiO}_{2} ; 1: 1\right.$ Petrol:Et $\left.\mathrm{t}_{2} \mathrm{O}\right)$ gave the title compound as a colourless oil (949 mg, 96\%). $\mathbf{R f} 0.31\left(\mathrm{Et}_{2} \mathrm{O}-\right.$ Petrol, 1:1); $[\boldsymbol{\alpha}]_{\mathbf{D}}{ }^{21}=-26.6\left(c=1.35, \mathrm{CH}_{3} \mathrm{OH}\right) ; \mathbf{I R} \boldsymbol{v}_{\max }($ neat $) / \mathrm{cm}^{-1} 1646(\mathrm{C}=\mathrm{O}) ;{ }^{1} \mathbf{H}$ NMR $\left(500 \mathrm{MHz}, \mathrm{CDCl}_{3}\right) \delta 7.22-7.11(\mathrm{~m}, 7 \mathrm{H}), 6.90(\mathrm{~d}, J=8.8 \mathrm{~Hz}, 2 \mathrm{H}), 5.45$ (q, $J=7.0$ $\mathrm{Hz}, 1 \mathrm{H}), 3.11$ (s, 3H), 2.12 (s, 3H), 1.32 (d, $J=7.1 \mathrm{~Hz}, 3 \mathrm{H}) ;{ }^{13} \mathbf{C}$ NMR $(125 \mathrm{MHz}$, $\left.\mathrm{CDCl}_{3}\right) \delta 161.7(\mathrm{C}), 145.5(\mathrm{C}), 140.7(\mathrm{C}), 129.6(\mathrm{C}), 129.5(\mathrm{CH}), 128.3(\mathrm{CH}), 127.2$ $(\mathrm{CH}), 127.2(\mathrm{CH}), 125.1(\mathrm{CH}), 53.8(\mathrm{CH}), 39.9\left(\mathrm{CH}_{3}\right), 30.7\left(\mathrm{CH}_{3}\right), 15.5\left(\mathrm{CH}_{3}\right)$; LRMS (ES+): $m / z 325\left(\mathrm{M}\left({ }^{35} \mathrm{Cl}\right)+\mathrm{Na}^{+}, 100\right), 327\left(\mathrm{M}\left({ }^{37} \mathrm{Cl}\right)+\mathrm{Na}^{+}, 30\right)$; HRMS (EI+): calcd for $\mathrm{C}_{17} \mathrm{H}_{19} \mathrm{O}_{1} \mathrm{~N}_{2}{ }^{35} \mathrm{Cl}_{1} \mathrm{Na}_{1}\left(\mathrm{M}+\mathrm{Na}^{+}\right)$: 325.1078 , found: 325.1072 [ $\left.\Delta-1.9 \mathrm{ppm}\right]$.

\section{1-(3-Chloro-4-fluorophenyl)-1,3-dimethyl-3-((S)-1-phenylethyl)urea 5i}

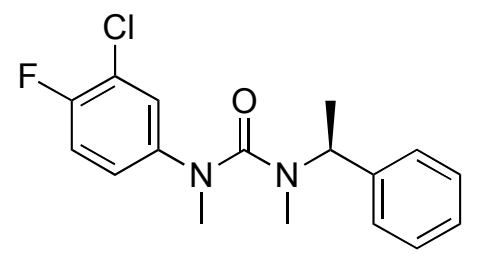

General procedures 1 and $2 \mathrm{~b}$ were followed. Flash column chromatography $\left(\mathrm{SiO}_{2} ; 1: 1\right.$ Petrol: $\mathrm{Et}_{2} \mathrm{O}$ ) gave the title compound as a colourless oil (389 mg, 78\%). Rf $0.29\left(\mathrm{Et}_{2} \mathrm{O}-\right.$ Petrol, 1:1); $[\boldsymbol{\alpha}]_{\mathbf{D}}{ }^{21}=-26.9\left(c=1.23, \mathrm{CH}_{3} \mathrm{OH}\right) ; \mathbf{I R} \boldsymbol{v}_{\max }($ neat $) / \mathrm{cm}^{-1} 1650(\mathrm{C}=\mathrm{O}) ;{ }^{1} \mathbf{H}$ NMR $\left(500 \mathrm{MHz}, \mathrm{CDCl}_{3}\right) \delta$ 7.24-7.21 (m, 2H), 7.18-7.13 (m, 3H), $7.06(\mathrm{dd}, J=6.4,2.7$ $\mathrm{Hz}, 1 \mathrm{H}), 6.97$ (t, $J=8.7 \mathrm{~Hz}, 1 \mathrm{H}$ ), 6.86 (ddd, $J=8.8,4.1,2.7 \mathrm{~Hz}, 1 \mathrm{H}), 5.44$ (q, $J=7.1$ $\mathrm{Hz}, 1 \mathrm{H}), 3.11(\mathrm{~s}, 3 \mathrm{H}), 2.16(\mathrm{~s}, 3 \mathrm{H}), 1.36(\mathrm{~d}, J=7.1 \mathrm{~Hz}, 3 \mathrm{H}) ;{ }^{13} \mathbf{C} \mathbf{N M R}(125 \mathrm{MHz}$, $\left.\mathrm{CDCl}_{3}\right) \delta 161.6(\mathrm{C}), 154.9\left(\mathrm{~d},{ }^{1} J_{\mathrm{CF}}=247.1 \mathrm{~Hz}, \mathrm{C}\right), 143.6\left(\mathrm{~d},{ }^{4} J_{\mathrm{CF}}=3.4 \mathrm{~Hz}, \mathrm{C}\right), 140.5(\mathrm{C})$, $128.4(\mathrm{CH}), 127.2$ (coupling obscured by nearby signals - possibly d, ${ }^{3} J_{\mathrm{CF}}=8.2 \mathrm{~Hz}, \mathrm{CH}$ ), 
$127.3(\mathrm{CH}), 126.0(\mathrm{CH}), 123.6\left(\mathrm{~d},{ }^{3} J_{\mathrm{CF}}=6.7 \mathrm{~Hz}, \mathrm{CH}\right), 121.4\left(\mathrm{~d},{ }^{2} J_{\mathrm{CF}}=18.5 \mathrm{~Hz}, \mathrm{C}\right), 117.1$ $\left(\mathrm{d},{ }^{2} J_{\mathrm{CF}}=22.2 \mathrm{~Hz}, \mathrm{CH}\right), 53.9(\mathrm{CH}), 40.1\left(\mathrm{CH}_{3}\right), 30.8\left(\mathrm{CH}_{3}\right), 15.5\left(\mathrm{CH}_{3}\right)$. LRMS (ES+): $m / z \quad 321 \quad\left(\mathrm{M}\left({ }^{35} \mathrm{Cl}\right)+\mathrm{H}^{+}, 100\right), \quad 323\left(\mathrm{M}\left({ }^{37} \mathrm{Cl}\right)+\mathrm{H}^{+}, 30\right)$; HRMS $(\mathrm{EI}+)$ : calcd for $\mathrm{C}_{17} \mathrm{H}_{19} \mathrm{O}_{1} \mathrm{~N}_{2}{ }^{35} \mathrm{Cl}_{1} \mathrm{~F}_{1}\left(\mathrm{M}+\mathrm{H}^{+}\right)$: 321.1164, found: 321.1171 [ $\Delta 2.0 \mathrm{ppm}$ ].

\section{1,3-Dimethyl-1-(naphthalen-1-yl)-3-((S)-1-phenylethyl)urea 5j}<smiles>C[C@H](c1ccccc1)N(C)C(=O)N(C)c1cccc2ccccc12</smiles>

General procedures 1 and $2 \mathrm{~b}$ were followed. Flash column chromatography $\left(\mathrm{SiO}_{2} ; 1: 1\right.$ Petrol:Et $\left.{ }_{2} \mathrm{O}\right)$ gave the title compound as a colourless oil $(711 \mathrm{mg}, 54 \%)$. $\mathbf{R f} 0.59\left(\mathrm{Et}_{2} \mathrm{O}-\right.$ Petrol, 1:1); $[\boldsymbol{\alpha}]_{\mathbf{D}}{ }^{21}=-12.4\left(c=2.31, \mathrm{CH}_{3} \mathrm{OH}\right) ; \mathbf{I R} \boldsymbol{v}_{\max }($ neat $) / \mathrm{cm}^{-1} 1636(\mathrm{C}=\mathrm{O}) ;{ }^{\mathbf{1}} \mathbf{H}$ NMR $\left(500 \mathrm{MHz}, \mathrm{CDCl}_{3}\right) \delta 7.88(\mathrm{~d}, J=8.4 \mathrm{~Hz}, 1 \mathrm{H}), 7.68(\mathrm{~d}, J=8.1 \mathrm{~Hz}, 1 \mathrm{H}), 7.54(\mathrm{~d}, J$ $=8.3 \mathrm{~Hz}, 1 \mathrm{H}), 7.37(\mathrm{ddd}, J=8.3,6.8,1.3 \mathrm{~Hz}, 1 \mathrm{H}), 7.31(\mathrm{ddd}, J=8.1,6.9,1.3 \mathrm{~Hz}, 1 \mathrm{H})$, $7.22(\mathrm{dd}, J=8.0,7.5 \mathrm{~Hz}, 1 \mathrm{H}), 7.08-7.05(\mathrm{~m}, 3 \mathrm{H}), 7.01-6.98(\mathrm{~m}, 3 \mathrm{H}), 5.37$ (q, $J=7.1 \mathrm{~Hz}$, $1 \mathrm{H}), 3.09$ (s, 3H), $1.82(\mathrm{~s}, 3 \mathrm{H}), 1.11(\mathrm{~d}, J=7.1 \mathrm{~Hz}, 3 \mathrm{H}) ;{ }^{13} \mathbf{C}$ NMR $\left(125 \mathrm{MHz}, \mathrm{CDCl}_{3}\right) \delta$ $163.3(\mathrm{C}), 143.2(\mathrm{C}), 141.1(\mathrm{C}), 134.8(\mathrm{C}), 129.6(\mathrm{C}), 128.6(\mathrm{CH}), 128.2(\mathrm{CH}), 127.2$ $(\mathrm{CH}), 126.9(\mathrm{CH}), 126.9(\mathrm{CH}), 126.8(\mathrm{CH}), 126.3(\mathrm{CH}), 125.8(\mathrm{CH}), 123.4(\mathrm{CH}), 122.8$ $(\mathrm{CH}), 53.8(\mathrm{CH}), 40.4\left(\mathrm{CH}_{3}\right), 30.3\left(\mathrm{CH}_{3}\right), 15.5\left(\mathrm{CH}_{3}\right)$; LRMS $(\mathrm{ES}+): \mathrm{m} / z 341\left(\mathrm{M}+\mathrm{Na}^{+}\right.$, 100), $319\left(\mathrm{M}+\mathrm{H}^{+}, 10\right)$; HRMS $(\mathrm{EI}+)$ : calcd for $\mathrm{C}_{21} \mathrm{H}_{23} \mathrm{O}_{1} \mathrm{~N}_{2}\left(\mathrm{M}+\mathrm{H}^{+}\right)$: 319.1805 , found: $319.1815[\Delta 3.2 \mathrm{ppm}]$.

\section{1,3-Dimethyl-1-(naphthalen-3-yl)-3-((S)-1-phenylethyl)urea 5k}

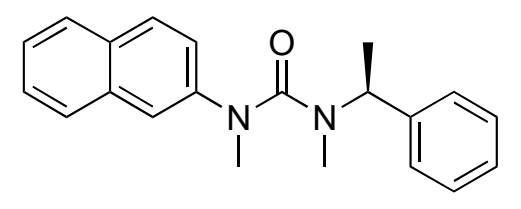

General procedures 1 and $2 \mathrm{~b}$ were followed. Flash column chromatography $\left(\mathrm{SiO}_{2} ; 1: 1\right.$ Petrol:Et $\left.{ }_{2} \mathrm{O}\right)$ gave the title compound as a colourless oil $(857 \mathrm{mg}, 91 \%)$. $\mathbf{R f} 0.28\left(\mathrm{Et}_{2} \mathrm{O}-\right.$ Petrol, 1:1); $[\boldsymbol{\alpha}]_{\mathbf{D}}{ }^{22}=-16.6\left(c=1.15, \mathrm{CH}_{3} \mathrm{OH}\right) ; \mathbf{I R} \boldsymbol{v}_{\max }($ neat $) / \mathrm{cm}^{-1} 1642(\mathrm{C}=\mathrm{O}) ;{ }^{1} \mathbf{H}$ NMR $\left(500 \mathrm{MHz}, \mathrm{CDCl}_{3}\right) \delta 7.72(\mathrm{~d}, J=8.8 \mathrm{~Hz}, 1 \mathrm{H}), 7.71(\mathrm{~d}, J=8.0 \mathrm{~Hz}, 1 \mathrm{H}), 7.63(\mathrm{~d}, J$ $=8.2 \mathrm{~Hz}, 1 \mathrm{H}), 7.41-7.38(\mathrm{~m}, 2 \mathrm{H}), 7.35(\mathrm{ddd}, J=8.1,6.9,1.3 \mathrm{~Hz}, 1 \mathrm{H}), 7.27-7.24(\mathrm{~m}$, 
$4 \mathrm{H}), 7.23(\mathrm{dd}, J=8.8,2.2 \mathrm{~Hz}, 1 \mathrm{H}),, 7.20-7.15(\mathrm{~m}, 1 \mathrm{H}), 5.60(\mathrm{q}, J=7.1 \mathrm{~Hz}, 1 \mathrm{H}), 3.29$ (s, 3H), $2.16(\mathrm{~s}, 3 \mathrm{H}), 1.38(\mathrm{~d}, J=7.1 \mathrm{~Hz}, 3 \mathrm{H}) ;{ }^{13} \mathbf{C}$ NMR $\left(125 \mathrm{MHz}, \mathrm{CDCl}_{3}\right) \delta 162.1(\mathrm{C})$, $144.3(\mathrm{C}), 140.9(\mathrm{C}), 133.9(\mathrm{C}), 130.6(\mathrm{C}), 129.4(\mathrm{CH}), 128.3(\mathrm{CH}), 127.6(\mathrm{CH}), 127.3$ $(\mathrm{CH}), 127.2(\mathrm{CH}), 127.1(\mathrm{CH}), 126.6(\mathrm{CH}), 125.4(\mathrm{CH}), 123.5(\mathrm{CH}), 120.8(\mathrm{CH}), 53.8$ $(\mathrm{CH}), 40.0\left(\mathrm{CH}_{3}\right), 30.8\left(\mathrm{CH}_{3}\right), 15.6\left(\mathrm{CH}_{3}\right)$; LRMS (ES+): $\mathrm{m} / z 341\left(\mathrm{M}+\mathrm{Na}^{+}, 100\right)$; HRMS (EI+): calcd for $\mathrm{C}_{21} \mathrm{H}_{23} \mathrm{O}_{1} \mathrm{~N}_{2}\left(\mathrm{M}+\mathrm{H}^{+}\right): 319.1805$, found: 319.1804 [ $\left.\Delta-0.3 \mathrm{ppm}\right]$.

\section{1-((R)-1-(4-Methoxyphenyl)ethyl)-1,3-dimethyl-3-phenylurea 51}

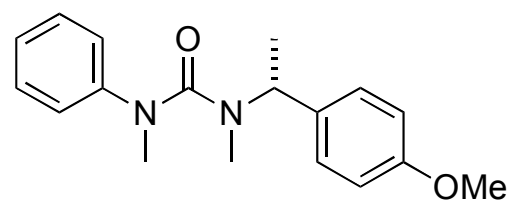

General procedures 1 and $2 \mathrm{~b}$ were followed. Flash column chromatography $\left(\mathrm{SiO}_{2} ; 1: 1\right.$ Petrol:Et $\left.{ }_{2} \mathrm{O}\right)$ gave the title compound as a colourless oil $(844 \mathrm{mg}, 86 \%)$. $\mathbf{R f} 0.48\left(\mathrm{Et}_{2} \mathrm{O}-\right.$ Petrol, 1:1); $[\boldsymbol{\alpha}]_{\mathbf{D}}{ }^{22}=+31.4\left(c=1.07, \mathrm{CH}_{3} \mathrm{OH}\right) ; \mathbf{I R} \boldsymbol{v}_{\max }($ neat $) / \mathrm{cm}^{-1} 1636(\mathrm{C}=\mathrm{O}) ;{ }^{1} \mathbf{H}$ NMR $\left(500 \mathrm{MHz}, \mathrm{CDCl}_{3}\right) \delta$ 7.21-7.18 (m, 2H), $7.11(\mathrm{~d}, J=8.4 \mathrm{~Hz}, 2 \mathrm{H}), 7.00-6.97(\mathrm{~m}$, $3 \mathrm{H}), 6.74(\mathrm{~d}, J=8.8 \mathrm{~Hz}, 2 \mathrm{H}), 5.42$ (q, $J=7.0 \mathrm{~Hz}, 1 \mathrm{H}), 3.67(\mathrm{~s}, 3 \mathrm{H}), 3.15(\mathrm{~s}, 3 \mathrm{H}), 2.09$ (s, 3H), $1.28(\mathrm{~d}, J=7.0 \mathrm{~Hz}, 3 \mathrm{H}) ;{ }^{13} \mathbf{C}$ NMR (125 MHz, $\left.\mathrm{CDCl}_{3}\right) \delta 162.0(\mathrm{C}), 158.6(\mathrm{C})$, $147.0(\mathrm{C}), 133.0(\mathrm{C}), 129.4(\mathrm{CH}), 128.4(\mathrm{CH}), 124.4(\mathrm{CH}), 124.1(\mathrm{CH}), 113.5(\mathrm{CH}), 55.2$ $\left(\mathrm{CH}_{3}\right), 53.2(\mathrm{CH}), 40.0\left(\mathrm{CH}_{3}\right), 30.4\left(\mathrm{CH}_{3}\right), 15.6\left(\mathrm{CH}_{3}\right)$; LRMS $(\mathrm{ES}+): m / z 321\left(\mathrm{M}+\mathrm{Na}^{+}\right.$, 100), $309\left(\mathrm{M}+\mathrm{H}^{+}, 10\right)$; HRMS (EI+): calcd for $\mathrm{C}_{18} \mathrm{H}_{22} \mathrm{O}_{2} \mathrm{~N}_{2} \mathrm{Na}_{1}\left(\mathrm{M}+\mathrm{Na}^{+}\right)$: 321.1573, found: 321.1578 [ $\Delta 1.4 \mathrm{ppm}]$.

\section{1-((S)-1-(4-Methoxyphenyl)ethyl)-1,3-dimethyl-3-o-tolylurea 5m}

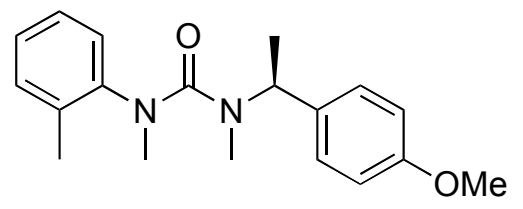

General procedures 1 and $2 \mathrm{~b}$ were followed. Flash column chromatography $\left(\mathrm{SiO}_{2} ; 1: 2\right.$ Petrol:Et $\mathrm{E}_{2} \mathrm{O}$ ) gave the title compound as a colourless oil (418 mg, 81\%). Rf 0.17 (Petrol:Et $\left.{ }_{2} \mathrm{O}, 1: 1\right) ;[\boldsymbol{\alpha}]_{\mathbf{D}}{ }^{22}=-49.0\left(c=1.20, \mathrm{CH}_{3} \mathrm{OH}\right) ; \mathbf{I R} \boldsymbol{v}_{\max }($ neat $) / \mathrm{cm}^{-1} 1636(\mathrm{C}=\mathrm{O}) ;{ }^{1} \mathbf{H}$ NMR $\left(500 \mathrm{MHz}, \mathrm{CDCl}_{3}\right) \delta 7.11(\mathrm{~d}, J=7.4 \mathrm{~Hz}, 1 \mathrm{H}), 7.08-7.00(\mathrm{~m}, 4 \mathrm{H}), 6.97(\mathrm{~d}, J=7.7$ 
$\mathrm{Hz}, 1 \mathrm{H}), 6.72$ (d, $J=8.8 \mathrm{~Hz}, 2 \mathrm{H}), 5.29$ (q, $J=7.0 \mathrm{~Hz}, 1 \mathrm{H}), 3.66$ (s, 3H), 2.99 (s, 3H), 2.19 (s, 3H), 1.98 (s, 3H), 1.18 (d, $J=7.0 \mathrm{~Hz}, 3 \mathrm{H}) ;{ }^{13} \mathbf{C}$ NMR (125 MHz, $\left.\mathrm{CDCl}_{3}\right) \delta 162.9$ (C), 158.5 (C), 145.5 (C), 134.2 (C), 133.2 (C), $131.5(\mathrm{CH}), 128.4(\mathrm{CH}), 127.1(\mathrm{CH})$, $126.6(\mathrm{CH}), 126.4(\mathrm{CH}), 113.5(\mathrm{CH}), 55.1\left(\mathrm{CH}_{3}\right), 53.3(\mathrm{CH}), 39.1\left(\mathrm{CH}_{3}\right), 29.7\left(\mathrm{CH}_{3}\right)$, $17.7\left(\mathrm{CH}_{3}\right), 15.5\left(\mathrm{CH}_{3}\right)$; LRMS (ES+): m/z $335\left(\mathrm{M}+\mathrm{Na}^{+}, 100\right), 313\left(\mathrm{M}+\mathrm{H}^{+}, 5\right)$; HRMS (EI+): calcd for $\mathrm{C}_{19} \mathrm{H}_{24} \mathrm{O}_{2} \mathrm{~N}_{2} \mathrm{Na}_{1}\left(\mathrm{M}+\mathrm{Na}^{+}\right)$: 335.1730 , found: 335.1729 [ $\left.\Delta-0.3 \mathrm{ppm}\right]$.

\section{1-((S)-1-(4-Methoxyphenyl)ethyl)-1,3-dimethyl-3-p-tolylurea 5n}

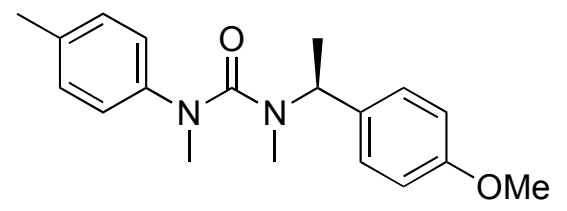

General procedures 1 and $2 \mathrm{~b}$ were followed. Flash column chromatography $\left(\mathrm{SiO}_{2} ; 1: 2\right.$ Petrol:Et ${ }_{2} \mathrm{O}$ ) gave the title compound as a colourless oil (413 mg, 80\%). Rf 0.17 (Petrol:Et $2 \mathrm{O}, 1: 1) ;[\boldsymbol{\alpha}]_{\mathbf{D}}{ }^{22}=-31.4\left(c=1.17, \mathrm{CH}_{3} \mathrm{OH}\right) ; \mathbf{I R} \boldsymbol{v}_{\max }($ neat $) / \mathrm{cm}^{-1} 1640(\mathrm{C}=\mathrm{O}) ;{ }^{1} \mathbf{H}$ NMR $\left(500 \mathrm{MHz}, \mathrm{CDCl}_{3}\right) \delta 7.19(\mathrm{~d}, J=8.4 \mathrm{~Hz}, 2 \mathrm{H}), 7.09(\mathrm{~d}, J=8.1 \mathrm{~Hz}, 2 \mathrm{H}), 6.97(\mathrm{~d}, J$ $=8.4 \mathrm{~Hz}, 2 \mathrm{H}), 6.83(\mathrm{~d}, J=8.8 \mathrm{~Hz}, 2 \mathrm{H}), 5.50(\mathrm{q}, J=7.1 \mathrm{~Hz}, 1 \mathrm{H}), 3.77(\mathrm{~s}, 3 \mathrm{H}), 3.20(\mathrm{~s}$, 3H), 2.29 (s, 3H), 2.16 (s, 3H), $1.36(\mathrm{~d}, J=7.1 \mathrm{~Hz}, 3 \mathrm{H}) ;{ }^{13} \mathbf{C ~ N M R}\left(125 \mathrm{MHz}, \mathrm{CDCl}_{3}\right) \delta$ 162.2 (C), 158.5 (C), 144.5 (C), 134.2 (C), 133.2 (C), $130.0(\mathrm{CH}), 128.4(\mathrm{CH}), 124.3$ $(\mathrm{CH}), 113.5(\mathrm{CH}), 55.2\left(\mathrm{CH}_{3}\right), 53.1(\mathrm{CH}), 40.3\left(\mathrm{CH}_{3}\right), 30.5\left(\mathrm{CH}_{3}\right), 20.8\left(\mathrm{CH}_{3}\right), 15.6$ $\left(\mathrm{CH}_{3}\right)$; LRMS (ES+): m/z $335\left(\mathrm{M}+\mathrm{Na}^{+}, 100\right), 313\left(\mathrm{M}+\mathrm{H}^{+}, 34\right)$; HRMS (EI+): calcd for $\mathrm{C}_{19} \mathrm{H}_{25} \mathrm{O}_{2} \mathrm{~N}_{2}\left(\mathrm{M}+\mathrm{H}^{+}\right): 313.1911$, found: 313.1908 [ $\left.\Delta-0.8 \mathrm{ppm}\right]$.

\section{1-((S)-1-(4-Methoxyphenyl)ethyl)-1,3-dimethyl-3-(naphthalen-1-yl)urea 50}<smiles>COc1ccc([C@H](C)N(C)C(=O)N(C)c2cccc3ccccc23)cc1</smiles>

General procedures 1 and $2 \mathrm{~b}$ were followed. Flash column chromatography $\left(\mathrm{SiO}_{2} ; 1: 2\right.$ Petrol:Et $\mathrm{E}_{2} \mathrm{O}$ ) gave the title compound as a colourless oil (455 mg, 79\%). Rf 0.17 (Petrol:Et $2 \mathrm{O}, 1: 1) ;[\boldsymbol{\alpha}]_{\mathbf{D}}{ }^{22}=-0.7\left(c=1.04, \mathrm{CH}_{3} \mathrm{OH}\right) ; \mathbf{I R} v_{\max }($ neat $) / \mathrm{cm}^{-1} 1640(\mathrm{C}=\mathrm{O}) ;{ }^{1} \mathbf{H}$ NMR $\left(500 \mathrm{MHz}, \mathrm{CDCl}_{3}\right) \delta 8.06(\mathrm{~d}, J=8.4 \mathrm{~Hz}, 1 \mathrm{H}), 7.86(\mathrm{~d}, J=7.9 \mathrm{~Hz}, 1 \mathrm{H}), 7.72(\mathrm{~d}, J$ 
$=8.3 \mathrm{~Hz}, 1 \mathrm{H}), 7.54(\mathrm{ddd}, J=8.3,6.8,1.3 \mathrm{~Hz}, 1 \mathrm{H}), 7.49(\mathrm{ddd}, J=8.0,6.9,1.2 \mathrm{~Hz}, 1 \mathrm{H})$, $7.41(\mathrm{dd}, J=8.1,7.4 \mathrm{~Hz}, 1 \mathrm{H}), 7.25(\mathrm{~d}, J=7.3 \mathrm{~Hz}, 1 \mathrm{H}), 7.12(\mathrm{~d}, J=8.4 \mathrm{~Hz}, 2 \mathrm{H}), 6.78$ (d, $J=8.8 \mathrm{~Hz}, 2 \mathrm{H}), 5.50$ (q, $J=7.0 \mathrm{~Hz}, 1 \mathrm{H}), 3.75$ (s, 3H), 3.26 (s, 3H), 1.99 (s, 3H), 1.26 (d, $J=7.0 \mathrm{~Hz}, 3 \mathrm{H}) ;{ }^{13} \mathbf{C}$ NMR $\left(125 \mathrm{MHz}, \mathrm{CDCl}_{3}\right) \delta 163.1(\mathrm{C}), 158.3(\mathrm{C}), 143.2(\mathrm{C}), 134.7$ (C), $133.0(\mathrm{C}), 129.4(\mathrm{C}), 128.5(\mathrm{CH}), 128.2(\mathrm{CH}), 126.7(\mathrm{CH}), 126.6(\mathrm{CH}), 126.1(\mathrm{CH})$, $125.6(\mathrm{CH}), 123.2(\mathrm{CH}), 122.7(\mathrm{CH}), 113.3(\mathrm{CH}), 55.0\left(\mathrm{CH}_{3}\right), 53.1(\mathrm{CH}), 40.2\left(\mathrm{CH}_{3}\right)$, $30.0\left(\mathrm{CH}_{3}\right), 15.4\left(\mathrm{CH}_{3}\right)$; LRMS (ES+): $m / z 371\left(\mathrm{M}+\mathrm{Na}^{+}, 100\right)$; HRMS (EI+): calcd for $\mathrm{C}_{22} \mathrm{H}_{24} \mathrm{O}_{2} \mathrm{~N}_{2} \mathrm{Na}_{1}\left(\mathrm{M}+\mathrm{Na}^{+}\right): 371.1730$, found: 371.1724 [ $\left.\Delta-1.6 \mathrm{ppm}\right]$.

\section{1-((R)-1-(4-Chlorophenyl)ethyl)-1,3-dimethyl-3-(naphthalen-1-yl)urea 5p}<smiles>C[C@H](c1ccc(Cl)cc1)N(C)C(=O)N(C)c1cccc2ccccc12</smiles>

General procedures 1 and $2 \mathrm{~b}$ were followed. Flash column chromatography $\left(\mathrm{SiO}_{2} ; 1: 1\right.$ Petrol: $\left.\mathrm{Et}_{2} \mathrm{O}\right)$ gave the title compound as a colourless oil $(1.13 \mathrm{~g}, 99 \%)$. $\mathbf{R f} 0.29\left(\mathrm{Et}_{2} \mathrm{O}-\right.$ Petrol, 1:1); $[\boldsymbol{\alpha}]_{\mathbf{D}}{ }^{21}=+0.8\left(c=1.97, \mathrm{CH}_{3} \mathrm{OH}\right) ; \mathbf{I R} \boldsymbol{v}_{\max }($ neat $) / \mathrm{cm}^{-1} 1636(\mathrm{C}=\mathrm{O}) ;{ }^{1} \mathbf{H}$ NMR $\left(500 \mathrm{MHz}, \mathrm{CDCl}_{3}\right) \delta 8.04(\mathrm{~d}, J=8.3 \mathrm{~Hz}, 1 \mathrm{H}), 7.85(\mathrm{~d}, J=8.1 \mathrm{~Hz}, 1 \mathrm{H}), 7.71(\mathrm{~d}, J=8.2$ $\mathrm{Hz}, 1 \mathrm{H}), 7.54$ (t, $J=7.4,7.4 \mathrm{~Hz}, 1 \mathrm{H}), 7.49$ (t, $J=7.3,7.3 \mathrm{~Hz}, 1 \mathrm{H}), 7.40$ (t, $J=7.8,7.8$ $\mathrm{Hz}, 1 \mathrm{H}), 7.24$ (d, $J=7.2 \mathrm{~Hz}, 1 \mathrm{H}), 7.19$ (d, $J=8.4 \mathrm{~Hz}, 2 \mathrm{H}), 7.10(\mathrm{~d}, J=8.4 \mathrm{~Hz}, 2 \mathrm{H}), 5.50$ (q, $J=7.0 \mathrm{~Hz}, 1 \mathrm{H}), 3.27$ (s, 3H), 1.99 (s, 3H), 1.27 (d, $J=7.0 \mathrm{~Hz}, 3 \mathrm{H}) ;{ }^{13} \mathbf{C}$ NMR (125 $\left.\mathrm{MHz}, \mathrm{CDCl}_{3}\right) \delta 163.1(\mathrm{C}), 143.0(\mathrm{C}), 139.6$ (C), 134.7 (C), 132.5 (C), 129.5 (C), 128.5 $(\mathrm{CH}), 128.4(\mathrm{CH}), 128.1(\mathrm{CH}), 126.8(\mathrm{CH}), 126.7(\mathrm{CH}), 126.2(\mathrm{CH}), 125.6(\mathrm{CH}), 123.3$ $(\mathrm{CH}), 122.6(\mathrm{CH}), 53.2(\mathrm{CH}), 40.2\left(\mathrm{CH}_{3}\right), 30.2\left(\mathrm{CH}_{3}\right), 15.4\left(\mathrm{CH}_{3}\right)$; LRMS (ES+): $\mathrm{m} / \mathrm{z}$ $353\left(\mathrm{M}\left({ }^{35} \mathrm{Cl}\right)+\mathrm{H}^{+}, 100\right), 355\left(\mathrm{M}\left({ }^{37} \mathrm{Cl}\right)+\mathrm{H}^{+}, 32\right)$; HRMS (EI+): calcd for $\mathrm{C}_{21} \mathrm{H}_{22} \mathrm{O}_{1} \mathrm{~N}_{2}{ }^{35} \mathrm{Cl}_{1}$ $\left(\mathrm{M}+\mathrm{H}^{+}\right): 353.1414$, found: 353.1415 [ $\left.\Delta 0.3 \mathrm{ppm}\right]$.

\section{1,3-Dimethyl-1-(1,1-diphenylethyl)urea 6a}

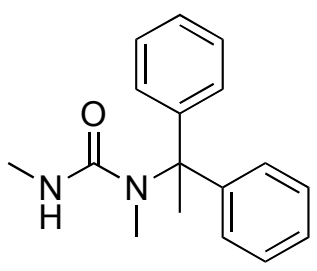


General procedure 3c was followed. Flash column chromatography $\left(\mathrm{SiO}_{2} ; 1: 1\right.$ Petrol:EtOAc) gave the title compound as a white solid (95 mg, 95\%). m.p. $182-183^{\circ} \mathrm{C}$ (Petrol-EtOAc); Rf 0.26 (EtOAc-Petrol-Et $\left.{ }_{3} \mathrm{~N}, 65: 32: 3\right) ; \mathbf{I R} v_{\max }\left(\mathrm{CHCl}_{3}\right) / \mathrm{cm}^{-1} 3312$ (NH), $1632(\mathrm{C}=\mathrm{O}) ;{ }^{1} \mathbf{H}$ NMR $\left(300 \mathrm{MHz}, \mathrm{CDCl}_{3}\right) \delta$ 7.40-7.21 (m, 10H), 3.98 (brs, $\left.1 \mathrm{H}\right), 2.98$ (s, 3H), $2.50(\mathrm{~d}, J=4 \mathrm{~Hz}, 3 \mathrm{H}), 2.11(\mathrm{~s}, 3 \mathrm{H}) ;{ }^{13} \mathbf{C}$ NMR $\left(75 \mathrm{MHz}, \mathrm{CDCl}_{3}\right) \delta 160.4(\mathrm{C})$, $145.3(\mathrm{C}), 128.9(\mathrm{CH}), 127.6(\mathrm{CH}), 127.5(\mathrm{CH}), 67.5(\mathrm{C}), 34.5\left(\mathrm{CH}_{3}\right), 31.6\left(\mathrm{CH}_{3}\right), 27.9$ $\left(\mathrm{CH}_{3}\right)$; LRMS m/z (CI+): $269\left(\mathrm{M}+\mathrm{H}^{+}, 45\right), 181$ (45), 89 (100); HRMS (EI+) calcd for $\mathrm{C}_{17} \mathrm{H}_{21} \mathrm{~N}_{2} \mathrm{O}\left(\mathrm{M}+\mathrm{H}^{+}\right): 269.1648$, found: 269.1647 [ $\Delta-0.3$ ppm]. EA found: $\mathrm{C} 76.24 \%, \mathrm{H}$ 7.67\%, N 10.07\%. $\mathrm{C}_{17} \mathrm{H}_{20} \mathrm{O}_{1} \mathrm{~N}_{2}$ requires C 76.09\%, H 7.51\%, N 10.49\%.

\section{1-((R)-1-(4-Methoxyphenyl)-1-phenylethyl)-1,3-dimethylurea 6b}

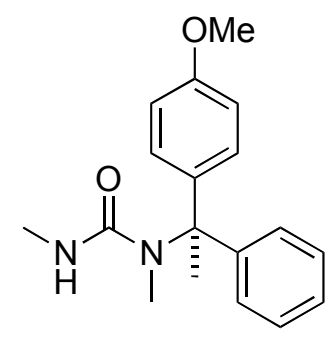

General procedure 3c was followed. Flash column chromatography $\left(\mathrm{SiO}_{2} ; 1: 1\right.$ Petrol:EtOAc) gave the title compound as a white foam $(82 \mathrm{mg}, 82 \%)$. Rf 0.23 (EtOAcPetrol, 2:1); $[\boldsymbol{\alpha}]_{\mathbf{D}}{ }^{23}=+8.2\left(c=1.17, \mathrm{CH}_{3} \mathrm{OH}\right) ; \mathbf{I R} \boldsymbol{v}_{\max }($ neat $) / \mathrm{cm}^{-1} 3350(\mathrm{~N}-\mathrm{H}), 1639$ $(\mathrm{C}=\mathrm{O}) ;{ }^{1} \mathbf{H}$ NMR $\left(500 \mathrm{MHz}, \mathrm{CD}_{3} \mathrm{OD}\right) \delta$ 7.24-7.21 (m, 2H), 7.19-7.17 (m, 2H), $7.15(\mathrm{t}, J$ $=7.1 \mathrm{~Hz}, 1 \mathrm{H}), 7.10(\mathrm{~d}, J=8.9 \mathrm{~Hz}, 2 \mathrm{H}), 6.79(\mathrm{~d}, J=8.9 \mathrm{~Hz}, 2 \mathrm{H}), 5.74(\mathrm{br}, 1 \mathrm{H}(\mathrm{NH}))$, 3.69 (s, 3H), 2.70 (s, 3H), 2.54 (s, 3H), 2.09 (s, 3H); $\left.{ }^{13} \mathbf{C ~ N M R ~ ( 1 2 5 ~ M H z , ~ C D ~}{ }_{3} \mathrm{OD}\right) \delta$ $162.1(\mathrm{C}), 159.7(\mathrm{C}), 147.6(\mathrm{C}), 139.1(\mathrm{C}), 129.5(\mathrm{CH}), 129.1(\mathrm{CH}), 127.9(\mathrm{CH}), 127.5$ $(\mathrm{CH}), 114.5(\mathrm{CH}), 68.6(\mathrm{C}), 55.7\left(\mathrm{CH}_{3}\right), 35.8\left(\mathrm{CH}_{3}\right), 30.3\left(\mathrm{CH}_{3}\right), 27.6\left(\mathrm{CH}_{3}\right)$; LRMS

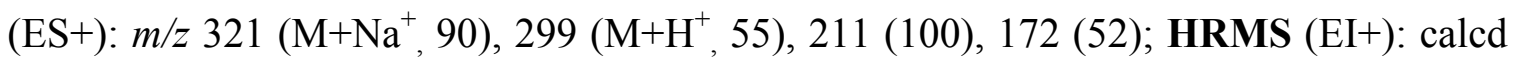
for $\mathrm{C}_{18} \mathrm{H}_{22} \mathrm{O}_{2} \mathrm{~N}_{2} \mathrm{Na}_{1}\left(\mathrm{M}+\mathrm{Na}^{+}\right): 321.1573$, found: 321.1566 [ $\left.\Delta-2.3 \mathrm{ppm}\right]$. 


\section{(R)-1-(1-(4-Methoxyphenyl)-1-phenylethyl)-1,3-dimethyl-3-nitrosourea 6b'}

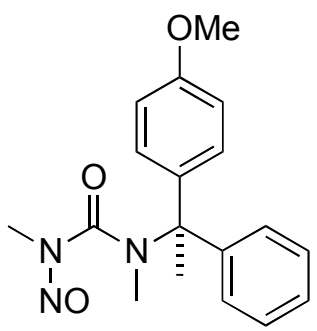

General procedure $3 \mathrm{~d}$ was followed. Flash column chromatography $\left(\mathrm{SiO}_{2} ; 1: 2\right.$ Petrol:EtOAc) gave the title compound as a pale yellow oil (57 mg, 46\%) [Racemic material rearranged in 65\% yield]. $\mathbf{R f} 0.45\left(\mathrm{Et}_{2} \mathrm{O}-\mathrm{Petrol}, 1: 1\right) ;[\boldsymbol{\alpha}]_{\mathbf{D}}{ }^{22}=+0.8(c=5.53$, $\left.\mathrm{CH}_{2} \mathrm{Cl}_{2}\right)$; IR $\boldsymbol{v}_{\max }($ neat $) / \mathrm{cm}^{-1} 1703(\mathrm{C}=\mathrm{O}) ;{ }^{1} \mathbf{H}$ NMR (500 MHz, CD $\left.{ }_{3} \mathrm{OD}\right) \delta$ 7.32-7.30 (m, 2H), 7.28-7.23 (m, 4H), 7.20-7.17 (m, 1H), $6.82(\mathrm{~d}, J=8.9 \mathrm{~Hz}, 2 \mathrm{H}), 3.72$ (s, 3H), 3.00 (s, 3H), 2.83 (s, 3H), 2.18 (s, 3H); ${ }^{13} \mathrm{C}$ NMR (125 MHz, CD $\left.{ }_{3} \mathrm{OD}\right) \delta 160.0(\mathrm{C}), 158.3$ (C), $145.8(\mathrm{C}), 137.2(\mathrm{C}), 129.7(\mathrm{CH}), 129.2(\mathrm{CH}), 128.0(\mathrm{CH}), 127.8(\mathrm{CH}), 114.5(\mathrm{CH}), 70.2$ (C), $55.7\left(\mathrm{CH}_{3}\right), 39.2\left(\mathrm{CH}_{3}\right), 29.4\left(\mathrm{CH}_{3}\right), 28.5\left(\mathrm{CH}_{3}\right)$. LRMS (ES+): $m / z 328\left(\mathrm{M}+\mathrm{H}^{+}, 10\right)$, 211 (100); HRMS (EI+): calcd for $\mathrm{C}_{18} \mathrm{H}_{22} \mathrm{O}_{3} \mathrm{~N}_{3}\left(\mathrm{M}+\mathrm{H}^{+}\right)$: 328.1656 , found: 328.1666 [ $\Delta$ $3.1 \mathrm{ppm}]$.

( \pm )-1-(1-(2-Methoxyphenyl)-1-phenylethyl)-1,3-dimethylurea 6c<smiles>CNC(=O)N(C)C(C)(c1ccccc1)c1ccccc1OC</smiles>

General procedure 3c was followed. Flash column chromatography $\left(\mathrm{SiO}_{2} ; 1: 1\right.$ Petrol:EtOAc) gave the title compound as a white crystalline solid (84 mg, 84\%). m.p 146-148 ${ }^{\circ} \mathrm{C}\left(\mathrm{CH}_{2} \mathrm{Cl}_{2}\right.$-Hexane); Rf 0.69 (EtOAc-Petrol, 2:1); IR $v_{\max }\left(\right.$ neat) $/ \mathrm{cm}^{-1} 3390$ (N$\mathrm{H}), 1637(\mathrm{C}=\mathrm{O}) ;{ }^{1} \mathbf{H}$ NMR (500 MHz, CD $\left.\mathrm{OD}\right) \delta$ 7.32-7.29 (m, 2H), 7.27-7.23 (m, 4H), $7.02(\mathrm{~d}, J=8.1 \mathrm{~Hz}, 1 \mathrm{H}), 6.80(\mathrm{t}, J=7.5 \mathrm{~Hz}, 1 \mathrm{H}), 6.75(\mathrm{dd}, J=1.6,7.8 \mathrm{~Hz}, 1 \mathrm{H}), 3.75(\mathrm{~s}$, 3H), 2.77 (s, 3H), 2.57 (s, 3H), 2.23 (s, 3H); ${ }^{13}$ C NMR (125 MHz, CD 3 OD) $\delta 161.9(\mathrm{C})$, $159.0(\mathrm{C}), 146.8(\mathrm{C}), 134.7(\mathrm{C}), 129.7(\mathrm{CH}), 129.4(\mathrm{CH}), 129.0(\mathrm{CH}), 128.7(\mathrm{CH}), 127.8$ $(\mathrm{CH}), 121.2(\mathrm{CH}), 113.1(\mathrm{CH}), 69.0(\mathrm{C}), 55.7\left(\mathrm{CH}_{3}\right), 35.2\left(\mathrm{CH}_{3}\right), 29.4\left(\mathrm{CH}_{3}\right), 27.6\left(\mathrm{CH}_{3}\right)$; LRMS (ES+): m/z $299\left(\mathrm{M}+\mathrm{H}^{+}, 100\right)$; HRMS (EI+): calcd for $\mathrm{C}_{18} \mathrm{H}_{23} \mathrm{O}_{2} \mathrm{~N}_{2}\left(\mathrm{M}+\mathrm{H}^{+}\right)$: 
299.1760, found: 299.1754 [ $\Delta-1.8 \mathrm{ppm}$ ]; EA found: C $72.00 \%, \mathrm{H} 7.61 \%$, N 9.34\%. $\mathrm{C}_{18} \mathrm{H}_{22} \mathrm{O}_{2} \mathrm{~N}_{2}$ requires C $72.46 \%, \mathrm{H} 7.43 \%, \mathrm{~N} 9.39 \%$.

\section{1-((S)-1-(3,4-Dimethoxyphenyl)-1-phenylethyl)-1,3-dimethylurea 6d}<smiles>CNC(=O)N(C)[C@](C)(c1ccccc1)c1ccc(OC)c(OC)c1</smiles>

General procedure $3 \mathrm{c}$ was followed. Flash column chromatography $\left(\mathrm{SiO}_{2} ; 1: 2\right.$ Petrol:EtOAc) gave the title compound as a white foam (76 mg, 76\%). Rf 0.27 (EtOAc); $[\boldsymbol{\alpha}]_{\mathbf{D}}{ }^{22}=+0.7\left(c=5.20, \mathrm{CH}_{3} \mathrm{OH}\right) ; \mathbf{I R} \boldsymbol{v}_{\max }($ neat $) / \mathrm{cm}^{-1} 3357(\mathrm{~N}-\mathrm{H}), 1639(\mathrm{C}=\mathrm{O}) ;{ }^{1} \mathbf{H} \mathbf{N M R}$ (500 MHz, $\left.\mathrm{CD}_{3} \mathrm{OD}\right) \delta$ 7.27-7.24 (m, 2H), 7.21-7.20 (m, 2H), 7.18-7.15 (m, 1H), 6.83$6.80(\mathrm{~m}, 2 \mathrm{H}), 6.71(\mathrm{dd}, J=8.5$ and $2.3 \mathrm{~Hz}, 1 \mathrm{H}), 5.80(\mathrm{~m}, 1 \mathrm{H}), 3.75(\mathrm{~s}, 3 \mathrm{H}), 3.70(\mathrm{~s}, 3 \mathrm{H})$, $2.73(\mathrm{~s}, 3 \mathrm{H}), 2.56(\mathrm{~s}, 3 \mathrm{H}), 2.12(\mathrm{~s}, 3 \mathrm{H}) ;{ }^{13} \mathrm{C}$ NMR (125 MHz, CD $\left.3 \mathrm{OD}\right) \delta 162.3(\mathrm{C}), 150.1$ (C), $149.4(\mathrm{C}), 147.5(\mathrm{C}), 140.1(\mathrm{C}), 129.2(\mathrm{CH}), 128.0(\mathrm{CH}), 127.6(\mathrm{CH}), 120.9(\mathrm{CH})$, $112.9(\mathrm{CH}), 112.3(\mathrm{CH}), 68.8(\mathrm{C}), 56.4\left(\mathrm{CH}_{3}\right), 56.4\left(\mathrm{CH}_{3}\right), 35.8\left(\mathrm{CH}_{3}\right), 30.2\left(\mathrm{CH}_{3}\right), 27.7$ $\left(\mathrm{CH}_{3}\right)$; LRMS (ES+): $m / z 241(78), 329\left(\mathrm{M}+\mathrm{H}^{+}, 85\right), 351\left(\mathrm{M}+\mathrm{Na}^{+}, 100\right)$; HRMS (EI+): calcd for $\mathrm{C}_{19} \mathrm{H}_{25} \mathrm{O}_{3} \mathrm{~N}_{2}\left(\mathrm{M}+\mathrm{H}^{+}\right)$: 329.1860, found: 329.1858 [ $\Delta-0.5 \mathrm{ppm}$ ].

\section{( \pm )-1-(1-(2-Fluorophenyl)-1-phenylethyl)-1,3-dimethylurea 6e}

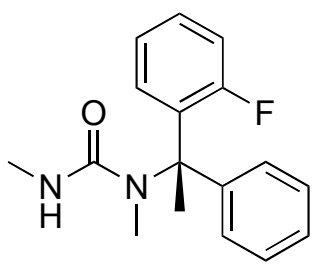

General procedure $3 \mathrm{c}$ was followed. Flash column chromatography $\left(\mathrm{SiO}_{2} ; 1: 2\right.$ Petrol:EtOAc) gave the title compound as a white crystalline solid (34 mg, 34\%). m.p. 151-153 ${ }^{\circ} \mathrm{C}(\mathrm{MeOH}) ; \mathbf{R f} 0.33$ (EtOAc-Petrol, 2:1); IR v $v_{\max }$ (neat) $/ \mathrm{cm}^{-1} 3366(\mathrm{~N}-\mathrm{H}), 1634$ $(\mathrm{C}=\mathrm{O}) ;{ }^{1} \mathbf{H}$ NMR $\left(500 \mathrm{MHz}, \mathrm{CD}_{3} \mathrm{OD}\right) \delta$ 7.28-7.25 (m, 2H), 7.20-7.17 (m, 4H), 7.01-6.96 $(\mathrm{m}, 2 \mathrm{H}), 6.86(\mathrm{td}, J=8.2,1.6 \mathrm{~Hz}, 1 \mathrm{H}), 2.71(\mathrm{~s}, 3 \mathrm{H}), 2.56(\mathrm{~s}, 3 \mathrm{H}), 2.14(\mathrm{~d}, J=2.0 \mathrm{~Hz}$, $3 \mathrm{H}) ;{ }^{13}$ C NMR (125 MHz, CD $\left.3 \mathrm{OD}\right) \delta 161.9(\mathrm{C}), 161.9\left(\mathrm{~d},{ }^{1} J_{\mathrm{CF}}=246.5 \mathrm{~Hz}, \mathrm{C}\right), 146.0$ 
(C), $134.3\left(\mathrm{~d},{ }^{2} J_{\mathrm{CF}}=11.6 \mathrm{~Hz}, \mathrm{C}\right), 130.1\left(\mathrm{~d},{ }^{3} J_{\mathrm{CF}}=9.9 \mathrm{~Hz}, \mathrm{CH}\right), 130.0\left(\mathrm{~d},{ }^{3} J_{\mathrm{CF}}=14.7 \mathrm{~Hz}\right.$, $\mathrm{CH}), 129.2(\mathrm{CH}), 128.4(\mathrm{CH}), 128.1(\mathrm{CH}), 124.9\left(\mathrm{~d},{ }^{4} J_{\mathrm{CF}}=3.5 \mathrm{~Hz}, \mathrm{CH}\right), 117.3\left(\mathrm{~d},{ }^{2} J_{\mathrm{CF}}=\right.$ $24.1 \mathrm{~Hz}, \mathrm{CH}), 68.1(\mathrm{C}), 35.3\left(\mathrm{CH}_{3}\right), 29.6\left(\mathrm{CH}_{3}\right), 27.6\left(\mathrm{CH}_{3}\right)$; LRMS (ES+): $m / z 287$ $\left(\mathrm{M}+\mathrm{H}^{+}, 25\right), 309\left(\mathrm{M}+\mathrm{Na}^{+}, 100\right)$; HRMS (EI+): calcd for $\mathrm{C}_{17} \mathrm{H}_{20} \mathrm{O}_{1} \mathrm{~N}_{2} \mathrm{~F}_{1}\left(\mathrm{M}+\mathrm{H}^{+}\right)$: 287.1554, found: 287.1555 [ $\Delta 0.3 \mathrm{ppm}]$.

( \pm )-1-(1-(3-Fluorophenyl)-1-phenylethyl)-1,3-dimethylurea $6 f$<smiles>CNC(=O)N(C)C(C)(c1ccccc1)c1cccc(F)c1</smiles>

General procedure $3 \mathrm{c}$ was followed. Flash column chromatography $\left(\mathrm{SiO}_{2} ; 1: 2\right.$ Petrol:EtOAc) gave the title compound as a white crystalline solid (40 mg, 40\%). m.p. 156-158 ${ }^{\circ} \mathrm{C}$ (MeOH); Rf 0.37 (EtOAc-Petrol, 2:1); IR $v_{\max }\left(\right.$ neat) $/ \mathrm{cm}^{-1} 1628(\mathrm{C}=\mathrm{O}) ;{ }^{1} \mathbf{H}$ NMR (500 MHz, $\left.\mathrm{CD}_{3} \mathrm{OD}\right) \delta 7.26-7.15(\mathrm{~m}, 6 \mathrm{H}), 7.03(\mathrm{~d}, J=8.0 \mathrm{~Hz}, 1 \mathrm{H}), 6.92$ (ddd, $J=$ 11.1, 2.2, $2.0 \mathrm{~Hz}, 1 \mathrm{H}), 6.87$ (td, $J=8.3,1.7 \mathrm{~Hz}, 1 \mathrm{H}), 6.07$ (br, NH), 2.72 (s, 3H), 2.57 (d, $J=4.4 \mathrm{~Hz}, 3 \mathrm{H}), 2.09(\mathrm{~s}, 3 \mathrm{H}) ;{ }^{13} \mathbf{C}$ NMR $\left(125 \mathrm{MHz}, \mathrm{CD}_{3} \mathrm{OD}\right) \delta 164.2\left(\mathrm{~d},{ }^{1} J_{\mathrm{CF}}=243.4\right.$ $\mathrm{Hz}, \mathrm{C}), 162.3(\mathrm{C}), 151.0$ (d, $\left.{ }^{3} J_{\mathrm{CF}}=6.9 \mathrm{~Hz}, \mathrm{C}\right), 146.8(\mathrm{C}), 130.7$ (d, $\left.{ }^{3} J_{\mathrm{CF}}=8.2 \mathrm{~Hz}, \mathrm{CH}\right)$, $129.3(\mathrm{CH}), 128.3(\mathrm{CH}), 127.9(\mathrm{CH}), 123.8\left(\mathrm{~d},{ }^{4} J_{\mathrm{CF}}=2.6 \mathrm{~Hz}, \mathrm{CH}\right), 115.0\left(\mathrm{~d},{ }^{2} J_{\mathrm{CF}}=23.2\right.$ $\mathrm{Hz}, \mathrm{CH}), 114.1\left(\mathrm{~d},{ }^{2} J_{\mathrm{CF}}=21.6 \mathrm{~Hz}, \mathrm{CH}\right), 68.9(\mathrm{C}), 35.9\left(\mathrm{CH}_{3}\right), 29.4\left(\mathrm{CH}_{3}\right), 27.7\left(\mathrm{CH}_{3}\right)$; LRMS (ES+): $m / z 287\left(\mathrm{M}+\mathrm{H}^{+}, 100\right)$; HRMS (EI+): calcd for $\mathrm{C}_{17} \mathrm{H}_{20} \mathrm{O}_{1} \mathrm{~N}_{2} \mathrm{~F}_{1}\left(\mathrm{M}+\mathrm{H}^{+}\right)$: 287.1554, found: 287.1548 [ $\Delta-2.2 \mathrm{ppm}]$.

\section{1-((S)-1-(4-Fluorophenyl)-1-phenylethyl)-1,3-dimethylurea 6g}

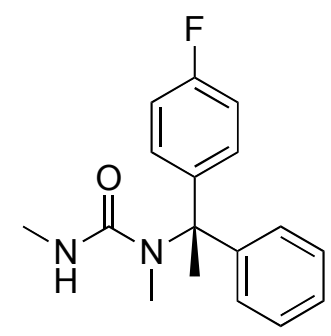

General procedure $3 \mathrm{c}$ was followed. Flash column chromatography $\left(\mathrm{SiO}_{2} ; 1: 2\right.$ Petrol:EtOAc) gave the title compound as a white crystalline solid (34 mg, 34\%). m.p. 
$160-162{ }^{\circ} \mathrm{C}(\mathrm{MeOH}) ; \mathbf{R f} 0.45$ (EtOAc-Petrol, 2:1); $[\boldsymbol{\alpha}]_{\mathbf{D}}{ }^{23}=+4.2\left(c=1.03, \mathrm{CH}_{3} \mathrm{OH}\right) ; \mathbf{I R}$ $v_{\max }($ neat $) / \mathrm{cm}^{-1} 1628(\mathrm{C}=\mathrm{O}) ;{ }^{1}$ H NMR $\left(500 \mathrm{MHz}, \mathrm{CD}_{3} \mathrm{OD}\right) \delta$ 7.25-7.13 (m, 7H), $6.95(\mathrm{t}$, $J=8.8 \mathrm{~Hz}, 2 \mathrm{H}), 2.70$ (s, 3H), 2.55 (s, 3H), 2.08 (s, 3H); ${ }^{13} \mathbf{C}$ NMR $\left(125 \mathrm{MHz}, \mathrm{CD}_{3} \mathrm{OD}\right) \delta$ $162.9\left(\mathrm{~d},{ }^{1} J_{\mathrm{CF}}=244.2 \mathrm{~Hz}, \mathrm{C}\right), 162.2(\mathrm{C}), 147.3(\mathrm{C}), 143.7\left(\mathrm{~d},{ }^{4} J_{\mathrm{CF}}=3.0 \mathrm{~Hz}, \mathrm{C}\right), 130.1(\mathrm{~d}$, $\left.{ }^{3} J_{\mathrm{CF}}=8.0 \mathrm{~Hz}, \mathrm{CH}\right), 129.3(\mathrm{CH}), 128.1(\mathrm{CH}), 127.8(\mathrm{CH}), 115.6\left(\mathrm{~d},{ }^{2} J_{\mathrm{CF}}=21.3 \mathrm{~Hz}, \mathrm{CH}\right)$, $68.7(\mathrm{C}), 35.9\left(\mathrm{CH}_{3}\right), 30.0\left(\mathrm{CH}_{3}\right), 27.6\left(\mathrm{CH}_{3}\right)$; LRMS (ES+): $\mathrm{m} / \mathrm{z} 287\left(\mathrm{M}+\mathrm{H}^{+}, 100\right)$; HRMS (EI+): calcd for $\mathrm{C}_{17} \mathrm{H}_{20} \mathrm{O}_{1} \mathrm{~N}_{2} \mathrm{~F}_{1}\left(\mathrm{M}+\mathrm{H}^{+}\right)$: 287.1554, found: $287.1545[\Delta-3.2$ ppm].

\section{1-((S)-1-(4-Chlorophenyl)-1-phenylethyl)-1,3-dimethylurea $6 \mathrm{~h}$}

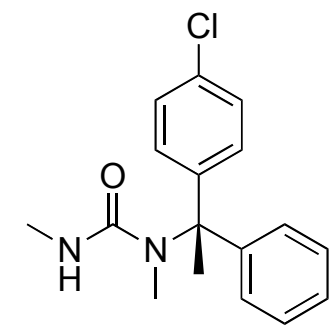

General procedure $3 \mathrm{c}$ was followed. Flash column chromatography $\left(\mathrm{SiO}_{2} ; 1: 2\right.$ Petrol:EtOAc) gave the title compound as a white crystalline solid (51 mg, 51\%). m.p. $155-157^{\circ} \mathrm{C}(\mathrm{MeOH}) ; \mathbf{R f} 0.32$ (EtOAc-Petrol, 2:1); $[\boldsymbol{\alpha}]_{\mathbf{D}}{ }^{23}=+3.1\left(c=1.01, \mathrm{CH}_{3} \mathrm{OH}\right) ; \mathbf{I R}$ $\nu_{\max }($ neat $) / \mathrm{cm}^{-1} 3312(\mathrm{~N}-\mathrm{H}), 1639(\mathrm{C}=\mathrm{O}) ;{ }^{1} \mathbf{H}$ NMR $\left(500 \mathrm{MHz}, \mathrm{CD}_{3} \mathrm{OD}\right) \delta$ 7.25-7.13 (m, 9H), 2.70 (s, 3H), 2.55 (s, 3H), 2.07 (s, 3H); ${ }^{13} \mathbf{C}$ NMR (125 MHz, $\left.\mathrm{CD}_{3} \mathrm{OD}\right) \delta 162.2(\mathrm{C})$, $146.9(\mathrm{C}), 146.7(\mathrm{C}), 133.1(\mathrm{C}), 129.6(\mathrm{CH}), 129.3(\mathrm{CH}), 129.0(\mathrm{CH}), 128.2(\mathrm{CH}), 127.8$ $(\mathrm{CH}), 68.7(\mathrm{C}), 35.8\left(\mathrm{CH}_{3}\right), 29.5\left(\mathrm{CH}_{3}\right), 27.6\left(\mathrm{CH}_{3}\right) ;$ LRMS $(\mathrm{ES}+): m / z 325$ $\left(\mathrm{M}\left({ }^{35} \mathrm{Cl}\right)+\mathrm{Na}^{+}, 100\right), 327\left(\mathrm{M}\left({ }^{37} \mathrm{Cl}\right)+\mathrm{Na}^{+}, 32\right)$; HRMS (EI+): calcd for $\mathrm{C}_{21} \mathrm{H}_{22} \mathrm{O}_{1} \mathrm{~N}_{2}{ }^{35} \mathrm{Cl}_{1}$ $\left(\mathrm{M}+\mathrm{H}^{+}\right)$: 353.14114 , found: 353.1415 [ $\left.\Delta 0.3 \mathrm{ppm}\right]$. 


\section{1-((S)-1-(3-Chloro-4-fluorophenyl)-1-phenylethyl)-1,3-dimethylurea $6 \mathbf{i}$}<smiles>CNC(=O)N(C)[C@@](C)(c1ccccc1)c1ccc(F)c(Cl)c1</smiles>

General procedure $3 \mathrm{c}$ was followed. Flash column chromatography $\left(\mathrm{SiO}_{2} ; 1: 2\right.$ Petrol:EtOAc) gave the title compound as a white crystalline solid (69 mg, 69\%). m.p. $127-129^{\circ} \mathrm{C}(\mathrm{MeOH}) ; \mathbf{R f} 0.52($ EtOAc-Petrol, $2: 1) ;[\boldsymbol{\alpha}]_{\mathbf{D}}{ }^{22}=+6.1\left(c=1.30, \mathrm{CH}_{3} \mathrm{OH}\right)$; IR $v_{\max }($ neat $) / \mathrm{cm}^{-1} 1629(\mathrm{C}=\mathrm{O}) ;{ }^{1} \mathbf{H}$ NMR $\left(500 \mathrm{MHz}, \mathrm{CD}_{3} \mathrm{OD}\right) \delta$ 7.27-7.24 (m, 3H), 7.21$7.14(\mathrm{~m}, 4 \mathrm{H}), 7.07(\mathrm{t}, J=8.9 \mathrm{~Hz}, 1 \mathrm{H}), 2.73(\mathrm{~s}, 3 \mathrm{H}), 2.58(\mathrm{~s}, 3 \mathrm{H}), 2.06(\mathrm{~s}, 3 \mathrm{H}) ;{ }^{13} \mathbf{C}$ NMR $\left(125 \mathrm{MHz}, \mathrm{CD}_{3} \mathrm{OD}\right) \delta 162.1(\mathrm{C}), 157.7\left(\mathrm{~d},{ }^{1} J_{\mathrm{CF}}=246.4 \mathrm{~Hz}, \mathrm{C}\right), 146.4(\mathrm{C}), 145.9\left(\mathrm{~d},{ }^{4} J_{\mathrm{CF}}\right.$ $=3.7 \mathrm{~Hz}, \mathrm{C}), 130.1(\mathrm{CH}), 129.4(\mathrm{CH}), 128.2(\mathrm{CH}), 128.1\left(\mathrm{~d},{ }^{3} J_{\mathrm{CF}}=10.9 \mathrm{~Hz}, \mathrm{CH}\right), 128.0$ $(\mathrm{CH}), 121.1\left(\mathrm{~d},{ }^{2} J_{\mathrm{CF}}=17.4 \mathrm{~Hz}, \mathrm{C}\right), 116.9\left(\mathrm{~d},{ }^{2} J_{\mathrm{CF}}=21.3 \mathrm{~Hz}, \mathrm{CH}\right), 68.3(\mathrm{C}), 35.6\left(\mathrm{CH}_{3}\right)$, $29.3\left(\mathrm{CH}_{3}\right), 27.6\left(\mathrm{CH}_{3}\right)\left[\left(1 \times 3 \mathrm{~J}_{\mathrm{CF}}\right.\right.$ obscured by nearby signals]; LRMS $(\mathrm{ES}+): \mathrm{m} / z 321$ $\left(\mathrm{M}\left({ }^{35} \mathrm{Cl}\right)+\mathrm{H}^{+}, 100\right), 323\left(\mathrm{M}\left({ }^{37} \mathrm{Cl}\right)+\mathrm{H}^{+}, 30\right)$; HRMS (EI+): calcd for $\mathrm{C}_{17} \mathrm{H}_{19} \mathrm{O}_{1} \mathrm{~N}_{2}{ }^{35} \mathrm{Cl}_{1} \mathrm{~F}_{1}$ $\left(\mathrm{M}+\mathrm{H}^{+}\right): 321.1164$, found: 321.1169 [ $\left.\Delta 1.4 \mathrm{ppm}\right]$.

\section{1,1,3-Trimethyl-3-((S)-1-(naphthalen-1-yl)-1-phenylethyl)urea 6j}<smiles>CN(C)C(=O)N(C)[C@@](C)(c1ccccc1)c1cccc2ccccc12</smiles>

Modified general procedures 3c was followed with MeI quench (10 equiv.) after $6 \mathrm{~h}$, the reaction allowed to warm to room temperature overnight, flash column chromatography $\left(\mathrm{SiO}_{2} ; 1: 1\right.$ Petrol:EtOAc) gave the title compound as a colourless oil (101 $\left.\mathrm{mg}, 88 \%\right)$. Rf $0.19\left(\mathrm{Et}_{2} \mathrm{O}-\mathrm{Petrol}, 1: 1\right) ;[\boldsymbol{\alpha}]_{\mathbf{D}}{ }^{23}=-56.3\left(c=4.65, \mathrm{CH}_{3} \mathrm{OH}\right) ; \mathbf{I R} \boldsymbol{v}_{\max }($ neat $) / \mathrm{cm}^{-1} 1648$ $(\mathrm{C}=\mathrm{O}) ;{ }^{1} \mathbf{H}$ NMR $\left(500 \mathrm{MHz}, \mathrm{CD}_{3} \mathrm{OD}\right) \delta 8.16(\mathrm{~d}, J=8.7 \mathrm{~Hz}, 1 \mathrm{H}), 7.88(\mathrm{~d}, J=8.0 \mathrm{~Hz}$, 1H), $7.78(\mathrm{~d}, J=8.1 \mathrm{~Hz}, 1 \mathrm{H}), 7.42-7.32(\mathrm{~m}, 7 \mathrm{H}), 7.29-7.27(\mathrm{~m}, 1 \mathrm{H}), 7.24(\mathrm{~d}, J=7.6 \mathrm{~Hz}$, 1H), 2.90 (s, 6H), 2.77 (s, 3H), 2.35 (s, 3H); ${ }^{13} \mathbf{C}$ NMR (75 MHz, CD $\left.{ }_{3} \mathrm{OD}\right) \delta 165.9$ (C), 146.2 (C), 141.9 (C), 136.4 (C), 132.4 (C), 130.3 (CH), 129.9 (CH), 129.3 (CH), 128.9 
$(\mathrm{CH}), 128.0(\mathrm{CH}), 127.8(\mathrm{CH}), 127.5(\mathrm{CH}), 125.9(\mathrm{CH}), 125.9(\mathrm{CH}), 125.7(\mathrm{CH}), 70.2$ $(\mathrm{CH}), 38.4\left(\mathrm{CH}_{3}\right), 38.2\left(\mathrm{CH}_{3}\right), 29.6\left(\mathrm{CH}_{3}\right)$.

\section{1,1,3-Trimethyl-3-((S)-1-(naphthalen-2-yl)-1-phenylethyl)urea 6k}

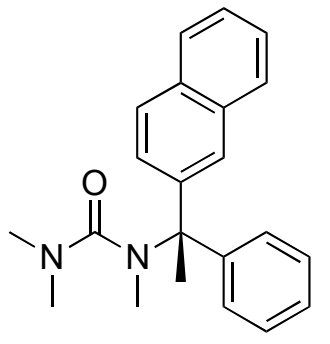

Modified general procedure 3c was followed with MeI quench (10 equiv.) after $6 \mathrm{~h}$. The reaction allowed to warm to room temperature overnight, and flash column chromatography $\left(\mathrm{SiO}_{2} ; 2: 1\right.$ Petrol:EtOAc) gave the title compound as a white crystalline solid (189 mg, 88\%). Rf 0.53 (EtOAc-Petrol, 2:1); m.p. $100-102{ }^{\circ} \mathrm{C}\left(\mathrm{CH}_{2} \mathrm{Cl}_{2}-\mathrm{Hexane}\right.$ ); $[\boldsymbol{\alpha}]_{\mathbf{D}}{ }^{22}=-52.1\left(c=1.22, \mathrm{CH}_{3} \mathrm{OH}\right) ; \mathbf{I R} \boldsymbol{v}_{\max }($ neat $) / \mathrm{cm}^{-1} 1648(\mathrm{C}=\mathrm{O}) ;{ }^{1} \mathbf{H} \mathbf{N M R}(500 \mathrm{MHz}$, $\left.\mathrm{CDCl}_{3}\right) \delta$ 7.68-7.62 (m, 4H), $7.46(\mathrm{dd}, J=8.7$ and $2.0 \mathrm{~Hz}, 1 \mathrm{H}), 7.33-7.27(\mathrm{~m}, 4 \mathrm{H}), 7.18$ (t, $J=7.8 \mathrm{~Hz}, 2 \mathrm{H}), 7.07$ (t, $J=7.3 \mathrm{~Hz}, 1 \mathrm{H}), 2.82(\mathrm{~s}, 6 \mathrm{H}), 2.59(\mathrm{~s}, 3 \mathrm{H}), 2.12(\mathrm{~s}, 3 \mathrm{H}) ;{ }^{13} \mathrm{C}$ NMR (125 MHz, $\left.\mathrm{CDCl}_{3}\right) \delta 160.0$ (C), 141.7 (C), 139.5 (C), 128.7 (C), 127.5 (C), 123.6 $(\mathrm{CH}), 123.5(\mathrm{CH}), 123.0(\mathrm{CH}), 122.7(\mathrm{CH}), 122.4(\mathrm{CH}), 121.6(\mathrm{CH}), 121.4(\mathrm{CH}), 121.3$ $(\mathrm{CH}), 121.3(\mathrm{CH}), 120.7(\mathrm{CH}), 62.6(\mathrm{C}), 33.2\left(\mathrm{CH}_{3}\right), 32.0\left(\mathrm{CH}_{3}\right), 20.2\left(\mathrm{CH}_{3}\right)$; LRMS (ES+): $m / z 333\left(\mathrm{M}+\mathrm{H}^{+}, 100\right)$; HRMS (EI+): calcd for $\mathrm{C}_{22} \mathrm{H}_{25} \mathrm{O}_{1} \mathrm{~N}_{2}\left(\mathrm{M}+\mathrm{H}^{+}\right)$: 333.1961, found: 333.1970 [ $\Delta 2.6 \mathrm{ppm}]$.

\section{1-((S)-1-(4-Methoxyphenyl)-1-o-tolylethyl)-1,3-dimethylurea 6m}

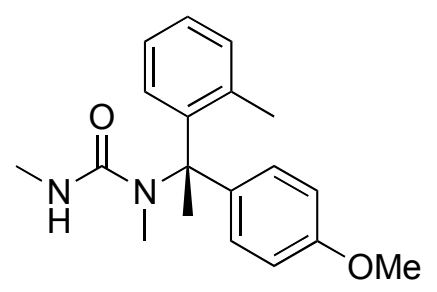

General procedures $3 \mathrm{c}$ was followed. Flash column chromatography $\left(\mathrm{SiO}_{2} ; 1: 2\right.$ Petrol:EtOAc) gave the title compound as a white foam $(64 \mathrm{mg}, 64 \%)$. Rf $0.25\left(\mathrm{Et}_{2} \mathrm{O}-\right.$ Petrol, 2:1); $[\boldsymbol{\alpha}]_{\mathbf{D}}{ }^{22}=-18.6\left(c=6.40, \mathrm{CH}_{3} \mathrm{OH}\right) ; \mathbf{I R} \boldsymbol{v}_{\max }($ neat $) / \mathrm{cm}^{-1} 3340(\mathrm{~N}-\mathrm{H}), 1634$ $(\mathrm{C}=\mathrm{O}) ;{ }^{1} \mathbf{H}$ NMR $\left(500 \mathrm{MHz}, \mathrm{CD}_{3} \mathrm{OD}\right) \delta$ 7.22-7.15 (m, 4H), $7.07(\mathrm{t}, J=7.6 \mathrm{~Hz}, 1 \mathrm{H}), 6.93$ 
(d, $J=9.0 \mathrm{~Hz}, 2 \mathrm{H}), 6.88(\mathrm{~d}, J=7.9 \mathrm{~Hz}, 1 \mathrm{H}), 3.83(\mathrm{~s}, 3 \mathrm{H}), 2.77(\mathrm{~s}, 3 \mathrm{H}), 2.65(\mathrm{~s}, 3 \mathrm{H}), 2.36$ (s, 3H), 2.26 (s, 3H); ${ }^{13} \mathrm{C}$ NMR (125 MHz, $\left.\mathrm{CD}_{3} \mathrm{OD}\right) \delta 162.0(\mathrm{C}), 160.1$ (C), 145.8 (C), $138.3(\mathrm{C}), 137.4(\mathrm{C}), 133.9(\mathrm{CH}), 130.4(\mathrm{CH}), 128.6(\mathrm{CH}), 128.0(\mathrm{CH}), 126.7(\mathrm{CH})$, $114.5(\mathrm{CH}), 69.6(\mathrm{C}), 55.8\left(\mathrm{CH}_{3}\right), 35.3\left(\mathrm{CH}_{3}\right), 30.7\left(\mathrm{CH}_{3}\right), 27.7\left(\mathrm{CH}_{3}\right), 22.7\left(\mathrm{CH}_{3}\right)$; LRMS (ES+): $m / z 225(100), 313\left(\mathrm{M}+\mathrm{H}^{+}, 60\right)$; HRMS (EI+): calcd for $\mathrm{C}_{19} \mathrm{H}_{25} \mathrm{O}_{2} \mathrm{~N}_{2}$ $\left(\mathrm{M}+\mathrm{H}^{+}\right): 313.1911$, found: $313.1905[\Delta-1.8 \mathrm{ppm}]$.

\section{1-((R)-1-(4-Methoxyphenyl)-1-p-tolylethyl)-1,3-dimethylurea 6n}

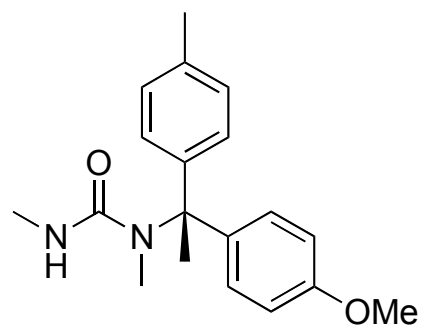

General procedure $3 \mathrm{c}$ was followed. Flash column chromatography $\left(\mathrm{SiO}_{2} ; 1: 2\right.$ Petrol:EtOAc) gave the title compound as a white foam (79 mg, 79\%). $\mathbf{R f} 0.23\left(\mathrm{Et}_{2} \mathrm{O}-\right.$ Petrol, 2:1); $[\alpha]_{\mathbf{D}}^{22}=+4.2\left(c=3.23, \mathrm{CH}_{3} \mathrm{OH}\right) ; \mathbf{I R} \boldsymbol{v}_{\max }$ (neat) $/ \mathrm{cm}^{-1} 3340(\mathrm{~N}-\mathrm{H}), 1635$ $(\mathrm{C}=\mathrm{O}) ;{ }^{1} \mathbf{H}$ NMR $\left(500 \mathrm{MHz}, \mathrm{CD}_{3} \mathrm{OD}\right) \delta 7.19(\mathrm{~d}, J=8.9 \mathrm{~Hz}, 2 \mathrm{H}), 7.16(\mathrm{~s}, 4 \mathrm{H}), 6.89(\mathrm{~d}, J$ $=8.9 \mathrm{~Hz}, 2 \mathrm{H}), 3.80(\mathrm{~s}, 3 \mathrm{H}), 2.80(\mathrm{~s}, 3 \mathrm{H}), 2.61(\mathrm{~s}, 3 \mathrm{H}), 2.35(\mathrm{~s}, 3 \mathrm{H}), 2.16(\mathrm{~s}, 3 \mathrm{H}) ;{ }^{13} \mathrm{C}$ NMR (125 MHz, CD 3 OD) $\delta 162.3$ (C), 159.8 (C), 144.5 (C), 139.3 (C), 137.4 (C), 129.8 $(\mathrm{CH}), 129.4(\mathrm{CH}), 128.0(\mathrm{CH}), 114.5(\mathrm{CH}), 68.4(\mathrm{C}), 55.7\left(\mathrm{CH}_{3}\right), 35.7\left(\mathrm{CH}_{3}\right), 30.5\left(\mathrm{CH}_{3}\right)$, $27.6\left(\mathrm{CH}_{3}\right), 20.9\left(\mathrm{CH}_{3}\right)$; LRMS (ES+): m/z 225 (100), $313\left(\mathrm{M}+\mathrm{H}^{+}, 18\right)$; HRMS (EI+): calcd for $\mathrm{C}_{19} \mathrm{H}_{25} \mathrm{O}_{2} \mathrm{~N}_{2}\left(\mathrm{M}+\mathrm{H}^{+}\right)$: 313.1911, found: 313.1917 [ $2.1 \mathrm{ppm}$ ].

\section{1-((R)-1-(4-Methoxyphenyl)-1-(naphthalen-1-yl)ethyl)-1,3,3-trimethylurea 6o}<smiles>COc1ccc([C@H](c2cccc3ccccc23)N(C)C(=O)N(C)C)cc1</smiles>

Modified general procedure 3c was followed with MeI quench (10 equiv.) after $6 \mathrm{~h}$ The reaction allowed to warm to room temperature overnight, and flash column chromatography $\left(\mathrm{SiO}_{2} ; 2: 1\right.$ Petrol:EtOAc) gave the title compound as a clear oil $(68 \mathrm{mg}$, 
$65 \%)$. Rf 0.48 (EtOAc-Petrol, 2:1); $[\boldsymbol{\alpha}]_{\mathbf{D}}{ }^{23}=+43.0\left(c=1.20, \mathrm{CH}_{3} \mathrm{OH}\right) ; \mathbf{I R} \boldsymbol{v}_{\max }($ neat $) / \mathrm{cm}^{-}$

${ }^{1} 1647(\mathrm{C}=\mathrm{O}) ;{ }^{1} \mathbf{H}$ NMR $\left(500 \mathrm{MHz}, \mathrm{CD}_{3} \mathrm{OD}\right) \delta 8.02(\mathrm{~d}, J=8.2 \mathrm{~Hz}, 1 \mathrm{H}), 7.80(\mathrm{~d}, J=7.2$ Hz, 1H), 7.70 (d, $J=8.1 \mathrm{~Hz}, 1 \mathrm{H}), 7.34-7.14(\mathrm{~m}, 7 \mathrm{H}), 6.82$ (d, $J=9.0 \mathrm{~Hz}, 2 \mathrm{H}), 3.74$ (s, 3H), 2.82 (s, 6H), 2.70 (s, 3H), 2.24 (s, 3H); ${ }^{13} \mathbf{C}$ NMR (125 MHz, $\left.\mathrm{CD}_{3} \mathrm{OD}\right) \delta 166.0$ (C), 159.9 (C), 138.1 (C), 136.5 (C), $132.4(\mathrm{C}), 131.2(\mathrm{C}), 131.2(\mathrm{CH}), 130.2(\mathrm{CH}), 129.2$ $(\mathrm{CH}), 128.2(\mathrm{CH}), 127.4(\mathrm{CH}), 125.9(\mathrm{CH}), 125.8(\mathrm{CH}), 125.6(\mathrm{CH}), 114.2(\mathrm{CH}), 69.8$ (C), $55.7\left(\mathrm{CH}_{3}\right), 38.4\left(\mathrm{CH}_{3}\right), 29.6\left(\mathrm{CH}_{3}\right), 25.3\left(\mathrm{CH}_{3}\right)$; LRMS (ES+): $m / z 363\left(\mathrm{M}+\mathrm{H}^{+}, 82\right)$, 261 (100); HRMS (EI+): calcd for $\mathrm{C}_{23} \mathrm{H}_{27} \mathrm{O}_{2} \mathrm{~N}_{2}\left(\mathrm{M}+\mathrm{H}^{+}\right)$: 363.2067 , found: 363.2059 [ $\Delta$ $-2.2 \mathrm{ppm}]$.

\section{1-((R)-1-(4-Chlorophenyl)-1-(naphthalen-1-yl)ethyl)-1,3,3-trimethylurea 6p}<smiles>CN(C)C(=O)N(C)[C@H](c1ccc(Cl)cc1)c1cccc2ccccc12</smiles>

Modified general procedure 3c was followed with MeI quench (10 equiv.) after $6 \mathrm{~h}$. The reaction allowed to warm to room temperature overnight, and flash column chromatography $\left(\mathrm{SiO}_{2} ; 2: 1\right.$ Petrol:EtOAc) gave the title compound as a clear oil (164 mg, $78 \%$ ). Rf 0.59 (EtOAc-Petrol, 1:2); $[\alpha]_{\mathbf{D}}{ }^{23}=+38.9\left(c=5.60, \mathrm{CH}_{3} \mathrm{OH}\right)$; IR $v_{\max }($ neat $) / \mathrm{cm}^{-}$ ${ }^{1} 1647(\mathrm{C}=\mathrm{O}) ;{ }^{1} \mathbf{H}$ NMR $\left(500 \mathrm{MHz}, \mathrm{CD}_{3} \mathrm{OD}\right) \delta 8.07(\mathrm{~d}, J=8.4 \mathrm{~Hz}, 1 \mathrm{H}), 7.78(\mathrm{~d}, J=8.1$ Hz, 1H), 7.69 (d, $J=8.1 \mathrm{~Hz}, 1 \mathrm{H}), 7.34-7.22(\mathrm{~m}, 7 \mathrm{H}), 7.10$ (d, $J=7.2 \mathrm{~Hz}, 1 \mathrm{H}), 2.80$ (s, 6H), 2.70 (s, 3H), 2.22 (s, 3H); ${ }^{13}$ C NMR (125 MHz, CD $\left.{ }_{3} \mathrm{OD}\right) \delta 165.7$ (C), 145.1 (C), $141.5(\mathrm{C}), 136.4(\mathrm{C}), 133.6(\mathrm{C}), 132.3(\mathrm{C}), 131.7(\mathrm{CH}), 130.4(\mathrm{CH}), 129.5(\mathrm{CH}), 128.9$ $(\mathrm{CH}), 127.7(\mathrm{CH}), 127.4(\mathrm{CH}), 126.0(\mathrm{CH}), 126.0(\mathrm{CH}), 125.9(\mathrm{CH}), 69.9(\mathrm{C}), 38.4$ $\left(\mathrm{CH}_{3}\right), 38.1\left(\mathrm{CH}_{3}\right), 29.4\left(\mathrm{CH}_{3}\right)$; LRMS (ES+): $m / z 367\left(\mathrm{M}\left({ }^{35} \mathrm{Cl}\right)+\mathrm{H}^{+}, 100\right), 369$ $\left(\mathrm{M}\left({ }^{37} \mathrm{Cl}\right)+\mathrm{H}^{+}, 37\right)$; HRMS (EI+): calcd for $\mathrm{C}_{22} \mathrm{H}_{24} \mathrm{O}_{1} \mathrm{~N}_{2}{ }^{35} \mathrm{Cl}_{1}\left(\mathrm{M}+\mathrm{H}^{+}\right)$: 367.1572, found: $367.1577[\Delta 1.5 \mathrm{ppm}]$. 
$\mathrm{N}$-Methyl-1,1-diphenylethanamine 7a

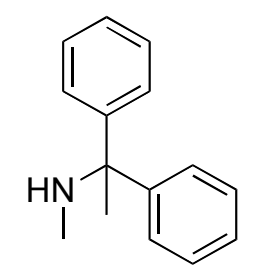

General procedure 4 was followed. Flash column chromatography $\left(\mathrm{SiO}_{2} ; 2: 1\right.$ Petrol:Et $\left.{ }_{2} \mathrm{O}\right)$ gave 1-(1,1-Diphenylethyl)-1,3-dimethyl-3-nitrosourea as a pale yellow solid (100 mg, 77\%). m.p. 114-116 ${ }^{\circ} \mathrm{C}$ (Et ${ }_{2} \mathrm{O}-P$ etrol); Rf 0.88 (EtOAc-Petrol, 2:1); IR $\nu_{\max }($ neat $) / \mathrm{cm}^{-1} 1703(\mathrm{C}=\mathrm{O}) ;{ }^{1} \mathbf{H}$ NMR $\left(500 \mathrm{MHz}, \mathrm{CD}_{3} \mathrm{OD}\right) \delta$ 7.33-7.32 (m, 4H), 7.29$7.26(\mathrm{~m}, 4 \mathrm{H}), 7.21-7.18(\mathrm{~m}, 2 \mathrm{H}), 3.00$ (s, 3H), 2.86 (s, 3H), 2.20 (s, 3H); ${ }^{13} \mathbf{C}$ NMR (125 $\left.\mathrm{MHz}, \mathrm{CD}_{3} \mathrm{OD}\right) \delta 158.5(\mathrm{C}), 145.4(\mathrm{C}), 129.3(\mathrm{CH}), 128.3(\mathrm{CH}), 128.1(\mathrm{CH}), 70.7(\mathrm{C})$, $39.3\left(\mathrm{CH}_{3}\right), 29.4\left(\mathrm{CH}_{3}\right), 28.4\left(\mathrm{CH}_{3}\right)$; LRMS (ES+): $m / z 181(100), 298\left(\mathrm{M}+\mathrm{H}^{+}, 46\right), 315$ $\left(\mathrm{M}+\mathrm{NH}_{4}{ }^{+}, 52\right)$; HRMS (EI+): calcd for $\mathrm{C}_{17} \mathrm{H}_{23} \mathrm{O}_{2} \mathrm{~N}_{4}\left(\mathrm{M}+\mathrm{NH}_{4}{ }^{+}\right)$: 315.1816, found: $315.1810[\Delta-1.8 \mathrm{ppm}]$.

General procedure 5 was followed. Flash column chromatography $\left(\mathrm{SiO}_{2} ; 1: 1\right.$ Petrol:EtOAc) gave the title compound as a colourless (80 mg, 94\%). Rf 0.5 (EtOAcPetrol, 2:1); IR v $v_{\max }($ neat $) / \mathrm{cm}^{-1} 1492 ;{ }^{1} \mathbf{H}$ NMR (500 MHz, $\left.\mathrm{CDCl}_{3}\right) \delta$ 7.24-7.23 (m, 4H), 7.20-7.17 (m, 4H), 7.11-7.09 (m, 2H), 2.10 (s, 3H), 1.68 (s, 3H); ${ }^{13}$ C NMR (100 MHz, $\left.\mathrm{CDCl}_{3}\right) \delta 147.8(\mathrm{C}), 127.8(\mathrm{CH}), 127.0(\mathrm{CH}), 126.2(\mathrm{CH}), 62.7(\mathrm{C}), 29.7\left(\mathrm{CH}_{3}\right), 26.7$ $\left(\mathrm{CH}_{3}\right)$; LRMS (ES+): $m / z 212\left(\mathrm{M}+\mathrm{H}^{+}, 23\right), 181(100)$; HRMS (EI+): calcd for $\mathrm{C}_{15} \mathrm{H}_{18} \mathrm{~N}_{1}$ $\left(\mathrm{M}+\mathrm{H}^{+}\right): 212.1434$, found: 212.1438 [ $\left.\Delta 2.0 \mathrm{ppm}\right]$.

\section{(R)-1-(4-Methoxyphenyl)- $N$-methyl-1-phenylethanamine $7 \mathrm{~b}$}

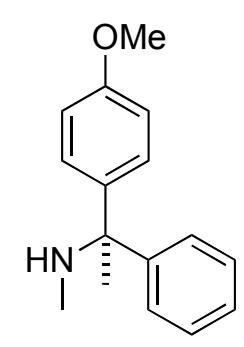

Nitroso-urea 6b' was treated according to general procedure 5. Flash column chromatography $\left(\mathrm{SiO}_{2} ; 1: 2\right.$ Petrol:EtOAc) gave the title compound as a colourless oil (21 $\mathrm{mg}, 53 \%$ ) [Racemic material gave a product in 82\% yield]. Rf 0.43 (EtOAc-Petrol, 2:1); 
$[\boldsymbol{\alpha}]_{\mathbf{D}}{ }^{22}=+4.8\left(c=2.14, \mathrm{CH}_{2} \mathrm{Cl}_{2}\right) ; \mathbf{I R} v_{\max }($ neat $) / \mathrm{cm}^{-1} 1509 ;{ }^{1} \mathbf{H}$ NMR $\left(500 \mathrm{MHz}, \mathrm{CD}_{3} \mathrm{OD}\right)$ $\delta$ 7.24-7.19 (m, 4H), $7.14(\mathrm{~d}, J=8.9 \mathrm{~Hz}, 2 \mathrm{H}), 7.14-7.11(\mathrm{~m}, 1 \mathrm{H}), 6.78(\mathrm{~d}, J=8.9 \mathrm{~Hz}$, 2H), 3.71 (s, 3H), 2.05 (s, 3H), $1.68(\mathrm{~s}, 3 \mathrm{H}) ;{ }^{13} \mathbf{C}$ NMR (125 MHz, $\left.\mathrm{CD}_{3} \mathrm{OD}\right) \delta 159.6(\mathrm{C})$, $149.0(\mathrm{C}), 140.5(\mathrm{C}), 129.4(\mathrm{CH}), 129.0(\mathrm{CH}), 128.1(\mathrm{CH}), 127.4(\mathrm{CH}), 114.3(\mathrm{CH}), 63.7$ (C), $55.6\left(\mathrm{CH}_{3}\right), 29.7\left(\mathrm{CH}_{3}\right), 27.0\left(\mathrm{CH}_{3}\right)$; LRMS (ES+): $m / z 242\left(\mathrm{M}+\mathrm{H}^{+}, 3\right), 211(100)$; HRMS (EI+): calcd for $\mathrm{C}_{16} \mathrm{H}_{20} \mathrm{O}_{1} \mathrm{~N}_{1}\left(\mathrm{M}+\mathrm{H}^{+}\right)$: 242.1539, found: 242.1544 [ $\Delta 1.9 \mathrm{ppm}$ ].

\section{(S)-1-(3-Chloro-4-fluorophenyl)- $N$-methyl-1-phenylethanamine 7i}

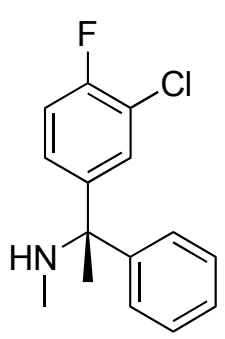

General procedure 4 was followed. Flash column chromatography $\left(\mathrm{SiO}_{2} ; 2: 1\right.$ Petrol:Et $\left.{ }_{2} \mathrm{O}\right)$ gave (S)-1-(1-(3-Chloro-4-fluorophenyl)-1-phenylethyl)-1,3-dimethyl-3nitrosourea as a white amorphous solid (43 $\mathrm{mg}, 81 \%)$. $\mathbf{R f} 0.48\left(\mathrm{Et}_{2} \mathrm{O}-\mathrm{Petrol}, 1: 1\right) ;[\boldsymbol{\alpha}]_{\mathbf{D}}{ }^{22}$ $=-3.0\left(c=1.17, \mathrm{CH}_{2} \mathrm{Cl}_{2}\right) ; \mathbf{I R} v_{\max }($ neat $) / \mathrm{cm}^{-1} 1702(\mathrm{C}=\mathrm{O}) ;{ }^{1} \mathbf{H} \mathbf{N M R}\left(500 \mathrm{MHz}, \mathrm{CD}_{2} \mathrm{Cl}_{2}\right)$ $\delta 7.40(\mathrm{dd}, J=7.0,2.5 \mathrm{~Hz}, 1 \mathrm{H}), 7.37-7.33(\mathrm{~m}, 4 \mathrm{H}), 7.30-7.27(\mathrm{~m}, 2 \mathrm{H}), 7.12(\mathrm{t}, J=8.8$ $\mathrm{Hz}, 1 \mathrm{H}), 3.07$ (s, 3H), 2.93 (s, 3H), 2.20 (s, 3H); ${ }^{13} \mathbf{C}$ NMR (125 MHz, $\left.\mathrm{CD}_{2} \mathrm{Cl}_{2}\right)$ $\delta 157.2\left(\mathrm{~d},{ }^{1} J_{\mathrm{CF}}=248.1 \mathrm{~Hz}, \mathrm{C}\right), 157.0(\mathrm{C}), 143.5(\mathrm{C}), 142.2\left(\mathrm{~d},{ }^{4} J_{\mathrm{CF}}=4.2 \mathrm{~Hz}, \mathrm{C}\right), 129.6$ $(\mathrm{CH}), 128.8(\mathrm{CH}), 127.6(\mathrm{CH}), 127.5\left(\mathrm{~d},{ }^{3} J_{\mathrm{CF}}=7.2 \mathrm{~Hz}, \mathrm{CH}\right), 127.2(\mathrm{CH}), 120.9\left(\mathrm{~d},{ }^{2} J_{\mathrm{CF}}\right.$ $=17.6 \mathrm{~Hz}, \mathrm{C}), 116.6\left(\mathrm{~d},{ }^{2} J_{\mathrm{CF}}=21.0 \mathrm{~Hz}, \mathrm{CH}\right), 68.8(\mathrm{C}), 38.6\left(\mathrm{CH}_{3}\right), 29.3\left(\mathrm{CH}_{3}\right), 27.9$ $\left(\mathrm{CH}_{3}\right) \quad\left(1 \times{ }^{3} \mathrm{~J}_{\mathrm{CF}}\right.$ coupling obscured by nearby signals); LRMS (ES+): $\mathrm{m} / \mathrm{z} 372$ $\left(\mathrm{M}\left({ }^{35} \mathrm{Cl}\right)+\mathrm{Na}^{+}, \quad 100\right), \quad 374 \quad\left(\mathrm{M}\left({ }^{37} \mathrm{Cl}\right)+\mathrm{Na}^{+}, \quad 31\right) ; \quad$ HRMS $(\mathrm{EI}+)$ : calcd for $\mathrm{C}_{17} \mathrm{H}_{17} \mathrm{O}_{2} \mathrm{~N}_{3} \mathrm{Cl}_{1} \mathrm{~F}_{1} \mathrm{Na}_{1}\left(\mathrm{M}\left({ }^{35} \mathrm{Cl}\right)+\mathrm{Na}^{+}\right): 372.0886$, found: 372.0895 [ $\Delta 2.5 \mathrm{ppm}$ ].

General procedure 5 was followed. Flash column chromatography $\left(\mathrm{SiO}_{2} ; 1: 1\right.$ Petrol:EtOAc) gave the title compound as a colourless oil ( $29 \mathrm{mg}$, 93\%). Rf 0.5 (EtOAcPetrol, 2:1); $[\boldsymbol{\alpha}]_{\mathbf{D}}{ }^{22}=+8.4\left(c=2.93, \mathrm{CH}_{2} \mathrm{Cl}_{2}\right) ; \mathbf{I R} \boldsymbol{v}_{\max }($ neat $) / \mathrm{cm}^{-1} 1494 ;{ }^{1} \mathbf{H}$ NMR (500 $\left.\mathrm{MHz}, \mathrm{CD}_{3} \mathrm{OD}\right) \delta 7.37(\mathrm{dd}, J=7.2$ and $2.4 \mathrm{~Hz}, 1 \mathrm{H}), 7.25-7.23(\mathrm{~m}, 4 \mathrm{H}), 7.17-7.14(\mathrm{~m}$, 2H), $7.07(\mathrm{t}, J=8.9 \mathrm{~Hz}, 1 \mathrm{H}), 2.06(\mathrm{~s}, 3 \mathrm{H}), 1.69(\mathrm{~s}, 3 \mathrm{H}) ;{ }^{13} \mathbf{C} \mathbf{N M R}\left(125 \mathrm{MHz}, \mathrm{CD}_{3} \mathrm{OD}\right) \delta$ $157.9\left(\mathrm{~d},{ }^{1} J_{\mathrm{CF}}=246.7 \mathrm{~Hz}, \mathrm{C}\right), 147.7(\mathrm{C}), 146.9\left(\mathrm{~d},{ }^{4} J_{\mathrm{CF}}=3.7 \mathrm{~Hz}, \mathrm{C}\right), 130.5(\mathrm{CH}), 129.3$ 
$(\mathrm{CH}), 128.7\left(\mathrm{~d},{ }^{3} J_{\mathrm{CF}}=7.1 \mathrm{~Hz}, \mathrm{CH}\right), 128.1(\mathrm{CH}), 127.8(\mathrm{CH}), 121.2\left(\mathrm{~d},{ }^{2} J_{\mathrm{CF}}=17.5 \mathrm{~Hz}\right.$, C), $117.0\left(\mathrm{~d},{ }^{2} J_{\mathrm{CF}}=20.9 \mathrm{~Hz}, \mathrm{CH}\right), 63.7(\mathrm{C}), 29.7\left(\mathrm{CH}_{3}\right), 26.7\left(\mathrm{CH}_{3}\right)\left(1 \times 3 J_{\mathrm{CF}}\right.$ coupling obscured by nearby signals); LRMS (ES+): $m / z 264\left(\mathrm{M}\left({ }^{35} \mathrm{Cl}\right)+\mathrm{H}^{+}, 43\right), 266\left(\mathrm{M}\left({ }^{37} \mathrm{Cl}\right)+\mathrm{H}^{+}\right.$, 13), 233 (100), 235 (30); HRMS (EI+): calcd for $\mathrm{C}_{15} \mathrm{H}_{16} \mathrm{~N}_{1} \mathrm{Cl}_{1} \mathrm{~F}_{1}\left(\mathrm{M}\left({ }^{35} \mathrm{Cl}\right)+\mathrm{H}^{+}\right)$: 264.0950, found: 264.0950 [ $\Delta 0.1 \mathrm{ppm}]$.

\section{(S)-N-Methyl-1-(naphthalen-1-yl)-1-phenylethanamine 7j}

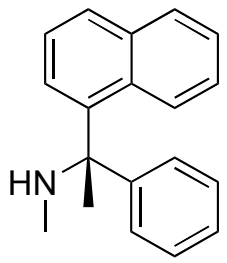

General procedure 4 was followed. Flash column chromatography $\left(\mathrm{SiO}_{2} ; 2: 1\right.$ Petrol:Et ${ }_{2} \mathrm{O}$ gave (S)-1,3-Dimethyl-1-(1-(naphthalen-1-yl)-1-phenylethyl)-3nitrosourea as a pale yellow oil $(70 \mathrm{mg}, 85 \%)$. $\mathbf{R f} 0.48\left(\mathrm{Et}_{2} \mathrm{O}-\mathrm{Petrol}, 1: 1\right) ;[\boldsymbol{\alpha}]_{\mathbf{D}}{ }^{22}=$ $+210.1\left(c=6.90, \mathrm{CH}_{2} \mathrm{Cl}_{2}\right) ;$ IR $v_{\max }($ neat $) / \mathrm{cm}^{-1} 1703(\mathrm{C}=\mathrm{O}) ;{ }^{1} \mathbf{H}$ NMR $(500 \mathrm{MHz}$, $\left.\mathrm{CD}_{2} \mathrm{Cl}_{2}\right) \delta 8.47(\mathrm{~d}, J=8.5 \mathrm{~Hz}, 1 \mathrm{H}), 7.87(\mathrm{dd}, J=7.9$ and $1.5 \mathrm{~Hz}, 1 \mathrm{H}), 7.76(\mathrm{~d}, J=8.2$ $\mathrm{Hz}, 1 \mathrm{H}), 7.54-7.38(\mathrm{~m}, 7 \mathrm{H}), 7.27(\mathrm{t}, J=7.8 \mathrm{~Hz}, 1 \mathrm{H}), 6.94(\mathrm{dd}, J=7.4$ and $0.9 \mathrm{~Hz}, 1 \mathrm{H})$, 3.07 (s, 3H), 3.05 (brs, 3H), 2.46 (s, 3H); ${ }^{13} \mathbf{C}$ NMR (125 MHz, $\left.\mathrm{CD}_{2} \mathrm{Cl}_{2}\right) \delta 156.6(\mathrm{C})$, $144.0(\mathrm{C}), 141.2(\mathrm{C}), 135.0(\mathrm{C}), 131.2(\mathrm{C}), 129.5(\mathrm{CH}), 128.9(\mathrm{CH}), 128.7(\mathrm{CH}), 128.6$ $(\mathrm{CH}), 127.8(\mathrm{CH}), 126.4(\mathrm{CH}), 126.3(\mathrm{CH}), 126.0(\mathrm{CH}), 125.6(\mathrm{CH}), 125.1(\mathrm{CH}), 71.1$ (C), $38.8\left(\mathrm{CH}_{3}\right), 29.3\left(\mathrm{CH}_{3}\right), 28.6\left(\mathrm{CH}_{3}\right)$.

General procedure 5 was followed. Flash column chromatography $\left(\mathrm{SiO}_{2} ; 1: 2\right.$ Petrol:EtOAc) gave the title compound as a colourless (47, 91\%). Rf 0.5 (EtOAc-Petrol, $2: 1) ;[\boldsymbol{\alpha}]_{\mathbf{D}}{ }^{22}=-88.3\left(c=4.70, \mathrm{CH}_{2} \mathrm{Cl}_{2}\right)$; IR $\boldsymbol{v}_{\max }($ neat $) / \mathrm{cm}^{-1} 2976 ;{ }^{1} \mathbf{H} \mathbf{~ N M R}(500 \mathrm{MHz}$, $\left.\mathrm{CD}_{3} \mathrm{OD}\right) \delta 7.76(\mathrm{~d}, J=7.3 \mathrm{~Hz}, 1 \mathrm{H}), 7.73(\mathrm{~d}, J=8.2 \mathrm{~Hz}, 2 \mathrm{H}), 7.58-7.55(\mathrm{~m}, 1 \mathrm{H}), 7.42(\mathrm{t}$, $J=7.8 \mathrm{~Hz}, 1 \mathrm{H}), 7.22-7.19(\mathrm{~m}, 3 \mathrm{H}), 7.13(\mathrm{t}, J=7.5 \mathrm{~Hz}, 2 \mathrm{H}), 7.07-7.05(\mathrm{~m}, 1 \mathrm{H}), 6.99$ (ddd, $J=8.4,6.9,1.2 \mathrm{~Hz}, 1 \mathrm{H}), 1.93$ (s, 3H), 1.80 (s, 3H); ${ }^{13} \mathbf{C}$ NMR (125 MHz, $\left.\mathrm{CD}_{3} \mathrm{OD}\right)$ \& $149.9(\mathrm{C}), 142.2(\mathrm{C}), 136.4(\mathrm{C}), 132.1(\mathrm{C}), 130.0(\mathrm{CH}), 129.6(\mathrm{CH}), 129.3(\mathrm{CH}), 127.7$ $(\mathrm{CH}), 127.3(\mathrm{CH}), 127.2(\mathrm{CH}), 126.6(\mathrm{CH}), 125.9(\mathrm{CH}), 125.9(\mathrm{CH}), 125.7(\mathrm{CH}), 64.6$ (C), $29.6\left(\mathrm{CH}_{3}\right), 28.7\left(\mathrm{CH}_{3}\right)$. LRMS (ES+): $\mathrm{m} / z 262\left(\mathrm{M}+\mathrm{H}^{+}, 80\right), 231$ (100); HRMS (EI+): calcd for $\mathrm{C}_{19} \mathrm{H}_{20} \mathrm{~N}_{1}\left(\mathrm{M}+\mathrm{H}^{+}\right): 262.1590$, found: 262.1588 [ $\Delta-0.9 \mathrm{ppm}$ ]. 
(1'R,5R)-5-(4-Methoxyphenyl)-1,3,5-trimethyl-4'H-spiro[imidazolidine-4,1'naphthalene]-2,4'-dione 100

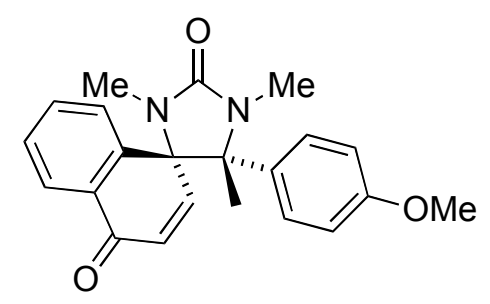

General procedure $3 \mathrm{e}$ was followed. Flash column chromatography $\left(\mathrm{SiO}_{2} ; 1: 2\right.$ Petrol:EtOAc) gave the title compound as a white crystalline solid (49 mg, 45\%). m.p $189-191{ }^{\circ} \mathrm{C}(\mathrm{MeOH}) ; \mathbf{R f} 0.32$ (EtOAc-Petrol, 2:1); $[\boldsymbol{\alpha}]_{\mathbf{D}}{ }^{22}=-373.8\left(c=0.95, \mathrm{CH}_{2} \mathrm{Cl}_{2}\right)$; IR $v_{\max }($ neat $) / \mathrm{cm}^{-1} 1703(\mathrm{C}=\mathrm{O}), 1700(\mathrm{C}=\mathrm{O}) ;{ }^{1} \mathbf{H}$ NMR $\left(500 \mathrm{MHz}, \mathrm{CD}_{2} \mathrm{Cl}_{2}\right) \delta 8.13(\mathrm{dd}, J$ $=7.7,1.2 \mathrm{~Hz}, 1 \mathrm{H}), 7.62(\mathrm{td}, J=7.8,1.3 \mathrm{~Hz}, 1 \mathrm{H}), 7.52(\mathrm{t}, J=7.1 \mathrm{~Hz}, 1 \mathrm{H}), 7.40(\mathrm{~d}, J=$ $7.9 \mathrm{~Hz}, 1 \mathrm{H}$ ), 6.97 (brs, 2H), 6.87 (brs, 1H), 6.86 (brs, 1H), 6.17 (d, $J=10.4 \mathrm{~Hz}, 1 \mathrm{H}), 6.08$ (d, $J=10.4 \mathrm{~Hz}, 1 \mathrm{H}), 3.80$ (s, 3H), 2.72 (s, 3H), 2.59 (s, 3H), 1.10 (s, 3H); ${ }^{13} \mathbf{C}$ NMR (125 $\left.\mathrm{MHz}, \mathrm{CD}_{2} \mathrm{Cl}_{2}\right) \delta 183.8(\mathrm{C}), 161.3(\mathrm{C}), 159.6(\mathrm{C}), 151.3(\mathrm{CH}), 140.4(\mathrm{C}), 133.2(\mathrm{C}), 132.7$ (C), $131.9(\mathrm{CH}), 131.1(\mathrm{CH}), 128.9(\mathrm{CH}), 127.5(\mathrm{CH}), 127.3(\mathrm{CH}), 69.9(\mathrm{C}), 68.1(\mathrm{C})$, $55.5\left(\mathrm{CH}_{3}\right), 27.7\left(\mathrm{CH}_{3}\right), 26.7\left(\mathrm{CH}_{3}\right), 21.9\left(\mathrm{CH}_{3}\right)(2 \times \mathrm{CH}$ signals missing due to broadening by slow rotation about Ar-C bond). LRMS (ES+): $m / z 363\left(\mathrm{M}+\mathrm{H}^{+}, 100\right)$; HRMS (EI+): calcd for $\mathrm{C}_{22} \mathrm{H}_{23} \mathrm{O}_{3} \mathrm{~N}_{1}\left(\mathrm{M}+\mathrm{H}^{+}\right)$: 263.1703, found: 263.1701 [ $\Delta-0.6 \mathrm{ppm}$ ].

\section{(1'R,5R)-5-(4-Chlorophenyl)-1,3,5-trimethyl-4'H-spiro[imidazolidine-4,1'-}

\section{naphthalene]-2,4'-dione $10 \mathrm{p}$}

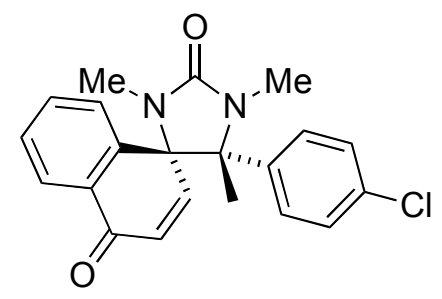

General procedure 3e was followed. Flash column chromatography $\left(\mathrm{SiO}_{2} ; 1: 2\right.$ Petrol:EtOAc) gave the title compound as a white crystalline solid (119 mg, 56\%). m.p $205-208{ }^{\circ} \mathrm{C}(\mathrm{MeOH}) ; \mathbf{R f} 0.50$ (EtOAc-Petrol, 2:1); $[\boldsymbol{\alpha}]_{\mathbf{D}}{ }^{22}=+344.1\left(c=2.34, \mathrm{CH}_{2} \mathrm{Cl}_{2}\right)$; IR $v_{\max }($ neat $) / \mathrm{cm}^{-1} 1702(\mathrm{C}=\mathrm{O}), 1699(\mathrm{C}=\mathrm{O}) ;{ }^{1} \mathbf{H}$ NMR $\left(500 \mathrm{MHz}, \mathrm{CD}_{2} \mathrm{Cl}_{2}\right) \delta 8.14(\mathrm{dd}, J$ 
$=7.7$ and $1.3 \mathrm{~Hz}, 1 \mathrm{H}), 7.64(\mathrm{td}, J=7.8$ and $1.5 \mathrm{~Hz}, 1 \mathrm{H}), 7.54(\mathrm{td}, J=7.6$ and $1.0 \mathrm{~Hz}$, 1H), 7.40 (d, $J=8.0 \mathrm{~Hz}, 1 \mathrm{H}$ ), 7.35 (brs, 1H), 7.33 (brs, 1H), 7.06 (brs, 1H), 6.96 (brs, $1 \mathrm{H}), 6.20(\mathrm{~d}, J=10.3 \mathrm{~Hz}, 1 \mathrm{H}), 6.06(\mathrm{~d}, J=10.4 \mathrm{~Hz}, 1 \mathrm{H}), 2.73(\mathrm{~s}, 3 \mathrm{H}), 2.59(\mathrm{~s}, 3 \mathrm{H}), 1.11$ (s, 3H); ${ }^{13}$ C NMR (125 MHz, $\left.\mathrm{CD}_{2} \mathrm{Cl}_{2}\right) \delta 183.6(\mathrm{C}), 161.2(\mathrm{C}), 150.6(\mathrm{CH}), 140.0(\mathrm{C})$, $138.9(\mathrm{C}), 134.2(\mathrm{C}), 133.1(\mathrm{C}), 132.9(\mathrm{CH}), 131.5(\mathrm{CH}), 129.2(\mathrm{CH}), 127.5(\mathrm{CH}), 127.4$ $(\mathrm{CH}), 69.9(\mathrm{C}), 67.9(\mathrm{C}), 27.8\left(\mathrm{CH}_{3}\right), 26.7\left(\mathrm{CH}_{3}\right), 21.8\left(\mathrm{CH}_{3}\right)(2 \times \mathrm{CH}$ signals missing due

to broadening by slow rotation about Ar-C bond). LRMS (ES+): $m / z 367\left(\mathrm{M}\left({ }^{35} \mathrm{Cl}\right)+\mathrm{H}^{+}\right.$, 100), $369\left(\mathrm{M}\left({ }^{37} \mathrm{Cl}\right)+\mathrm{H}^{+}, 37\right), 384(82), 386(25)$; HRMS (EI+): calcd for $\mathrm{C}_{21} \mathrm{H}_{20} \mathrm{O}_{2} \mathrm{~N}_{2}{ }^{35} \mathrm{Cl}_{1}$ $\left(\mathrm{M}+\mathrm{H}^{+}\right): 367.1208$, found: $367.1199[\Delta-2.4 \mathrm{ppm}]$.

\section{References}

1. W. C. Still, M. Kahn, A. Mitra, J. Org. Chem., 1978, 43, 2923.

2. W. L. F. Armarego, D. D. Perrin, Purification of Laboratory Chemicals, Pergamon Press, 1988.

3. J. Clayden, H. Turner, M. Pickworth, T. Adler, Org. Lett., 2005, 7, 3147

4. J. Leonard, B. Lygo, G. Procter, Advanced Practical Organic Chemistry, second ed., Nelson Thornes (Publishers) Ltd, 2004, pp. 178.

5. A. Padwa, W. Bergmark, D. Pashayan, J. Am. Chem. Soc., 1969, 91, 2653.

6. S. Cicchi, M. Bonanni, F. Cardona, J. Revuelta, A. Goti, Org. Lett., 2003, 5, 1773.

7. O. Cervinka, V. Dudek. Collect. Czech. Chem. Commun., 1973, 38, 1159. 